\title{
Propriedades genéricas de equilíbrios de equações parabólicas
}

\author{
Daniel Wellichan Mancini
}

Orientador: Prof. Dr. José Gaspar Ruas Filho

Dissertação apresentada ao Instituto de Ciências Matemáticas e de Computação - ICMC-USP, como parte dos requisitos para obtenção do titulo de Mestre em Matematica.

USP - São Carios

Agosto/2001 
A Comissão Julgadora:

Prof. Dr. José Gaspar Ruas Filho

Prof. Dr. Alexandre Nolasco de Carvalho

Prof. Dr. Adalberto Panobianco Bergamasco
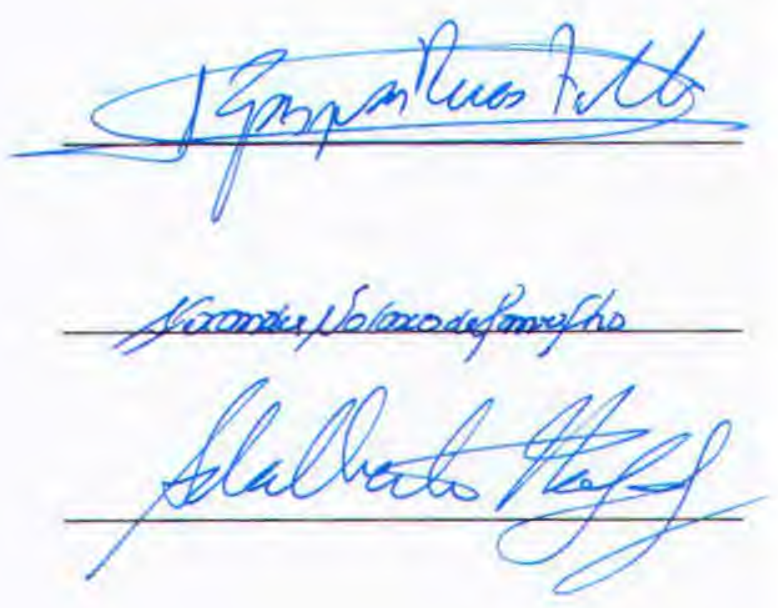
À Carolina 


\section{Agradecimentos $^{1}$}

Agradeço a todos aqueles que contribuíram, sob as mais variadas formas, para a conclusão deste trabalho. Em especial, registro minha gratidão ao Prof. José Gaspar Ruas Filho, pela paciência e generosidade com que tem me assitido e orientado desde o início de meus est udos na graduação.

${ }^{1}$ Este trabalho teve suporte financeiro da FAPESP 


\section{Resumo}

Nesta dissertação estudaremos a hiperbolicidade genérica dos equilíbrios de equações parabólicas da forma

$$
\begin{aligned}
& u_{t}=\Delta u+f(x, u, \nabla u), t>0, x \in \Omega \\
& u=0, t>0, x \in \partial \Omega .
\end{aligned}
$$

Primeiramente, fixada uma função suave $f$, mostraremos que todos os seus equilíbrios são hiperbólicos quando a região $\Omega$ percorre um conjunto residual de uma classe de domínios regulares. Depois, fixada uma região regular $\Omega$, suporemos que $f$ independe de $\nabla u$ e provaremos que todos os equilíbrios são hiperbólicos quando $f$ varia em um subconjunto residual de um conjunto de funções suficientemente regulares. Para a obtenção destes dois resultados utilizamos uma generalização, obtida por D. Henry, do Teorema da Transversalidade, cuja demonstração apresentamos neste trabalho. 


\section{Abstract}

In this dissertation we study the generic hyperbolicity of the equilibria of parabolic equations in the form

$$
\begin{aligned}
& u_{t}=\Delta u+f(x, u, \nabla u), t>0, x \in \Omega \\
& u=0, t>0, x \in \partial \Omega
\end{aligned}
$$

First, we fix a smooth function $f$ and show that all equilibria of this equation are hyperbolic when the domain $\Omega$ runs over a residual set in a certain class of regular domains. Then, we fix a region $\Omega$ and, supposing that $f$ does not depend on $\nabla u$, we proof that all equilibria are hyperbolic when $f$ belongs to a residual subset in a set of sufficiently smooth functions. To obtain these two results we use a generalization, due to D. Henry, of the Transversality Theorem, whose proof we present in this work. 


\section{Sumário}

Agradecimentos $\quad$ i

Resumo $\quad$ iii

Abstract $\quad$ v

$\begin{array}{ll}\text { Introduçāo } & 1\end{array}$

1 Preliminares 3

2 Operadores de Fredholm $\quad 9$

3 Perturbação de Contorno $\quad 21$

3.1 Cálculo Diferencial das Perturbações de Fronteira . . . . . . . . . . . . . 21

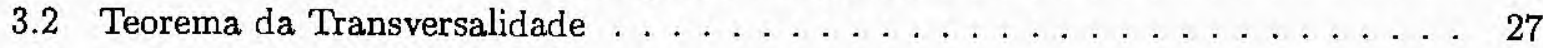

4 Propriedades Genéricas de Equilíbrios de Equações Parabólicas 41

4.1 Simplicidade Genérica das Soluçōes de $\Delta u+f(x, u)=0 \ldots \ldots \ldots \ldots \ldots$

4.2 Simplicidade Genérica dos Autovalores Reais . . . . . . . . . . . . . . 46

4.3 Simplicidade Genérica das Soluções de $\Delta u+f(x, u, \nabla u)=0 \ldots \ldots \ldots$

$\begin{array}{ll}\text { Bibliografia } & \mathbf{5 7}\end{array}$ 


\section{Introdução}

Nesta dissertação estudaremos a hiperbolicidade genérica dos equilíbrios de equações parabólicas sob duas perspectivas distintas, refletidas basicamente em dois resultados.

Primeiramente, fixado um domínio $\Omega \subset \mathbb{R}^{n}$, limitado e com fronteira $C^{3}$-regular, consideramos o problema de Dirichlet para a equação de reação-difusão

$$
\begin{aligned}
& u_{t}=\Delta u+f(x, u), t>0, x \in \Omega \\
& u=0, t>0, x \in \partial \Omega
\end{aligned}
$$

em que $f: \Omega \times \mathbb{R} \rightarrow \mathbb{R}$ é uma funçāo de classe $C^{k}(k \geq 2)$. Provaremos que todos os equilíbrios da equação acima são hiperbólicos qualquer que seja a função $f$ de classe $C^{k}$ exceto, possivelmente, para $f$ escolhida em um subconjunto magro (segundo a topologia de Whitney) do espaço das funções de classe $C^{k}$.

Em seguida, fixada uma função $f: \mathbb{R}^{n} \times \mathbb{R} \times \mathbb{R}^{n} \rightarrow \mathbb{R}$ de classe $C^{3}$, nos dedicamos ao estudo da equação

$$
\begin{aligned}
& u_{t}=\Delta u+f(x, u, \nabla u), t>0, x \in \Omega \\
& u=0, t>0, x \in \partial \Omega
\end{aligned}
$$

com o objetivo de verificar que todos os seus equilíbrios são hiperbólicos quando $\Omega$ percorre o complementar de um subconjunto magro da classe das regiōes abertas, limitadas, conexas e $C^{3}$-regulares.

Neste último caso, baseamo-nos no trabalho desenvolvido em [12] por D. Henry, que dispensou especial atenção ao problema de perturbações da fronteira, introduzindo novas técnicas e ferramentas, entre as quais se destaca uma generalização do Teorema da Transversalidade enunciado por Abraham e Thom [1] essencial na obtenção do segundo resultado.

No Capítulo 1 são apresentadas definições, fixadas notações e expostos alguns resultados preliminares referentes a operadores diferenciais em hipersuperfícies, operadores elípticos e imersões de espaços de Sobolev.

O Capítulo 2 reservamos para o estudo dos operadores de Fredholm entre espaços de Banach, sobretudo os limitados (objetos presentes muito freqüentemente no restante do trabalho), 
provando propriedades que serão úteis nos capítulos subsequentes.

Ocupamo-nos no Capítulo 3 com a exposição de técnicas desenvolvidas por D. Henry em [12] para abordar a questão das perturbações de fronteira em problemas de contorno, mostrando um tipo de cálculo diferencial envolvendo tais perturbaçōes e a demonstração da versão generalizada do Teorema da Transversalidade a que nos referimos anteriormente.

Por fim, reunidos nos três primeiros capítulos os instrumentos adequados para o estudo da hiperbolicidade génerica dos equilíbrios, no Capıtulo 4 tratamos os dois problemas apresentados nos parágrafos iniciais desta introdução. 


\section{Capítulo 1}

\section{Preliminares}

Sejam $E$ um espaço vetorial normado, $U \subset \mathbb{R}^{n}$ um conjunto aberto e $f: U \longrightarrow E$ uma aplicação $m$-vezes diferenciável em $x \in U$. Podemos considerar a $m$-ésima derivada de $f$ em $x, D^{m} f(x)$, como uma forma $m$-linear simétrica sobre $\mathbb{R}^{n}$

$$
\begin{gathered}
D^{m} f(x): \mathbb{R}^{n} \times \ldots \times \mathbb{R}^{n} \longrightarrow E \\
D^{m} f(x) \cdot v_{1} \ldots v_{k}=\frac{\partial^{m} f}{\partial v_{m} \partial v_{m-1} \cdots \partial v_{1}}(x)
\end{gathered}
$$

ou como a coleção de derivadas parciais

$$
D^{m} f(x)=\left\{\left(\frac{\partial}{\partial x}\right)^{\alpha} f(x) ;|\alpha|=m\right\}
$$

onde o multi-índice $\alpha$ é uma $n$-upla $\alpha=\left(\alpha_{1}, \ldots, \alpha_{n}\right)$ de inteiros não-negativos, $|\alpha|=\alpha_{1}+\cdots+\alpha_{n}$ e

$$
\left(\frac{\partial}{\partial x}\right)^{\alpha}=\frac{\partial^{\alpha_{n}}}{\partial x_{n}^{\alpha_{n}}} \ldots \frac{\partial^{\alpha_{1}}}{\partial x_{1}^{\alpha_{1}}}
$$

Às vezes, escrevemos $D^{\alpha}$ em lugar de $\left(\frac{\partial}{\partial x}\right)^{\alpha}$.

Desse modo, a norma $\left|D^{m} f(x)\right|$ pode significar

$$
\max _{|\alpha|=m}\left\|\left(\frac{\partial}{\partial x}\right)^{\alpha} f(x)\right\| \quad \text { ou } \max _{|h| \leq 1}\left\|D^{m} f(x) h^{m}\right\|,
$$

segundo a interpretação adotada para $D^{m} f(x)$.

Se $\Omega$ é um aberto de $\mathbb{R}^{n}$, indicaremos sua fronteira por $\partial \Omega$ e seu fecho por $\bar{\Omega}$. Dado um inteiro $m \geq 0$ e considerando ainda $E$ um espaço vetorial normado, denotaremos por $C^{m}(\Omega, E)$ o espaço das aplicações $f: \Omega \longrightarrow E$ limitadas, $m$-vezes diferenciáveis, com derivadas contínuas e limitadas que se estendem continuamente ao fecho $\bar{\Omega}$, munido da norma usual

$$
\|f\|_{C^{m}(\Omega, E)}=\max _{0 \leq j \leq m} \sup _{x \in \Omega}\left|D^{j} f(x)\right| .
$$

$C_{0}^{m}(\Omega, E)$ indicará o subespaço de $C^{m}(\Omega, E)$ consistindo de todas as funções $f \in C^{m}(\Omega, E)$ com suporte compacto. 
$C_{u n i f}^{m}(\Omega, E)$ denotará o subsepaço fechado de $C^{m}(\Omega, E)$ consistindo das funçōes cuja $m$-ésima derivada é uniformemente contínua. Observe que $C_{u n i f}^{m}(\Omega, E)$ e $C^{m}(\Omega, E)$ coincidem quando $\Omega$ é limitado.

$C^{m, \alpha}(\Omega, E)$ é o subespaço de $C_{u n i f}^{m}(\Omega, E)$ formado pelas aplicações cuja $m$-ésima derivada é Hölder contínua com expoente $\alpha, 0<\alpha \leq 1$, provido da norma

$$
\|f\|_{C^{m, \alpha}(\Omega, E)}=\max \left\{\|f\|_{C^{m}(\Omega, E)}, H_{\alpha}^{\Omega}\left(D^{m} f\right)\right\}
$$

em que

$$
H_{\alpha}^{\Omega}(f)=\sup \left\{|f(x)-f(y)| /|x-y|^{\alpha} ; x \neq y \in \Omega\right\} .
$$

No caso em que $E=\mathbb{R}$ omitimos o espaço $E$, escrevendo apenas $C^{m}(\Omega), C_{0}^{m}(\Omega)$, etc.

Dizemos que um aberto $\Omega \subset \mathbb{R}^{n}$ é $C^{m}$-regular $\left[C^{m, \alpha}\right.$-regular $]-$ ou que $\Omega$ tem fronteira

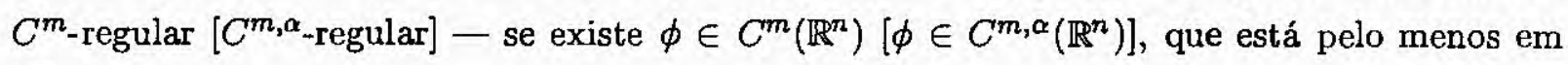
$C_{\text {unif }}^{1}\left(\mathbb{R}^{n}\right)$, tal que

$$
\Omega=\left\{x \in \mathbb{R}^{n} ; \phi(x)>0\right\}
$$

e $\phi(x)=0$ implica que $|\nabla \phi(x)| \geq 1$

D. Henry demonstra em [12] que esta definição de região $C^{m}$-regular equivale àquela usada por Agmon-Douglis-Nirenberg [3] em seu artigo sobre problemas de contorno para operadores elípticos.

Se $m \geq 1$ é um inteiro e $p$ é um número real com $1 \leq p \leq \infty$, denotamos por $W^{m, p}(\Omega)$ o espaço das funções $u \in L^{p}(\Omega)$ que têm todas as suas derivadas fracas de ordem $\leq m$ em $L^{p}(\Omega)$, ou seja,

$$
\begin{gathered}
W^{m, p}(\Omega)=\left\{u \in L^{p}(\Omega) ; \text { para todo } \alpha \text { com }|\alpha| \leq m, \text { existe } D^{\alpha} u \in L^{p}(\Omega)\right. \\
\text { tal que } \left.\int_{\Omega} u D^{\alpha} \phi=(-1)^{|\alpha|} \int_{\Omega} D^{\alpha} u \phi, \forall \phi \in C_{0}^{1}(\Omega)\right\} .
\end{gathered}
$$

$\operatorname{Em} W^{m, p}(\Omega)$ consideramos a norma

$$
\|u\|_{W^{m, p}(\Omega)}=\sum_{0 \leq|\alpha| \leq m}\left\|D^{\alpha} u\right\|_{L^{p}(\Omega)}
$$

Se $1 \leq p<\infty, W_{0}^{m, p}(\Omega)$ designará o fecho de $C_{0}^{m}(\Omega)$ em $W^{m, p}(\Omega)$.

Precisaremos, em algumas circunstâncias, utilizar operadores diferenciais em uma hipersuperfície $S \subset \mathbb{R}^{n}$. As definições que inserimos a seguir sāo intrínsecas a $S$ e independem do ambiente $\mathbb{R}^{n}$.

Sejam $S$ uma hipersuperfície de classe $C^{1}$ em $\mathbb{R}^{n}$ e $\varphi: S \rightarrow \mathbb{R}$ uma aplicação de classe $C^{1}$ (de modo que $\varphi$ pode ser estendida a uma aplicação de classe $C^{\mathfrak{l}}$ em uma vizinhança de $S$ ). 
Definimos $\nabla_{S} \varphi$ como o campo vetorial tangente sobre $S$ tal que para cada curva $x(t) \subset S$ de classe $C^{1}$ temos

$$
\frac{d}{d t} \varphi(x(t))=\left(\nabla_{S} \varphi\right)(x(t)) \cdot x^{\prime}(t)
$$

Sejam $S$ uma hipersuperfície de classe $C^{2}$ em $\mathbb{R}^{n}$ e $\vec{a}: S \longrightarrow \mathbb{R}^{n}$ um campo vetorial de classe $C^{1}$. Então $\operatorname{div}_{S} \vec{a}: S \longrightarrow \mathbb{R}$ é a função contínua tal que, para cada funçâo $\varphi: S \longrightarrow \mathbb{R}$ de classe $C^{1}$ com suporte compacto em $S$,

$$
\int_{S}\left(\operatorname{div}_{S} \vec{a}\right) \varphi=-\int_{S} \vec{a} \nabla_{S} \varphi
$$

Por fim, se $u: S \longrightarrow \mathbb{R}$ é de classe $C^{2}$, entâo $\Delta_{S} u=\operatorname{div}\left(\nabla_{S} u\right)$ ou, equivalentemente, é a função contínua que satisfaz

$$
\int_{S} \varphi \Delta_{S} u=-\int_{S} \nabla_{S} \varphi \cdot \nabla_{S} u
$$

No teorema abaixo estão reunidos alguns fatos relacionados aos objetos definidos acima.

Teorema 1.1. (1) Se S é uma hipersuperfície de classe $C^{1}$ e $\varphi: \mathbb{R}^{n} \longrightarrow \mathbb{R}$ é uma aplicação $C^{1}$ em uma vizinhança de $S$, entâo

$$
\nabla_{S} \varphi(x)=\nabla \varphi(x)-\frac{\partial \varphi}{\partial N}(x) \cdot N,
$$

onde $N$ é um campo vetorial normal unitário sobre $S$.

(2) Sejam $S$ uma hipersuperfície de classe $C^{2}, \vec{a}: \mathbb{R}^{n} \longrightarrow \mathbb{R}^{n}$ um campo vetorial de classe $C^{1}$ em uma vizinhança de $S, N: \mathbb{R}^{n} \longrightarrow \mathbb{R}^{n}$ um campo vetorial unitário de classe $C^{1}$ em uma vizinhança de $S$, que é um campo normal a $S$ sobre os pontos de $S$. Entâo

$$
\operatorname{div}_{S} \vec{a}=\operatorname{div} \vec{a}-(\operatorname{div} N) \vec{a} \cdot N-\frac{\partial \vec{a}}{\partial N} \cdot N .
$$

(3) Sejam $S$ uma hipersuperfície de classe $C^{2}, u: \mathbb{R}^{n} \rightarrow \mathbb{R}$ um funçāo de classe $C^{2}$ em uma vizinhança de $S$ e $N$ um campo vetorial unitário normal para $S$, como em (2). Então

$$
\Delta_{S} u=\Delta u-\operatorname{div} N \frac{\partial u}{\partial N}-\frac{\partial^{2} u}{\partial N^{2}}+\nabla_{S u} \cdot \frac{\partial N}{\partial N}
$$

sobre $S$.

Os próximos resultados, com os quais finalizamos este capítulo reservado aos pré-requisitos, tratam de operadores elípticos e podem ser encontrados em [8], [2] e [13]. Serão utilizados com frequência no Capítulo 4 .

Vamos considerar operadores $L$ da forma

$$
u \longmapsto L u=\sum_{i, j}^{n} a_{i j}(x) \frac{\partial^{2} u}{\partial x_{i} \partial x_{j}}+\sum_{i=1}^{n} b_{i}(x) \frac{\partial u}{\partial x_{i}}+c(x) u,
$$


com coeficientes $a_{i j}=a_{j i}, b_{i}, c, i, j=1, \ldots, n$, definidos em um domínio $\Omega \subset \mathbb{R}^{n}$.

Dizemos que $L$ é elíptico em um ponto $x \in \Omega$ se a matriz dos coeficientes $\left[a_{i j}(x)\right]$ é positiva, ou seja, se $\lambda(x)$ e $\Lambda(x)$ denotam o menor e o maior autovalores de $\left[a_{i j}(x)\right]$, respectivamente, então

$$
0<\lambda(x)|\xi|^{2} \leq \sum_{i, j=1}^{n} a_{i j} \xi_{i} \xi_{j} \leq \Lambda(x)|\xi|^{2},
$$

para todo $\xi=\left(\xi_{1}, \ldots, \xi_{n}\right) \in \mathbb{R}^{n} \backslash\{0\}$.

Se $\lambda(x) \geq 0$ para todo $x \in \Omega$ então $L$ é elíptico em $\Omega$. Quando, para alguma constante $\lambda_{0}$, temos $\lambda(x) \geq \lambda_{0}>0$ qualquer que seja $x \in \Omega$, dizemos que $L$ é estritamente elíptico em $\Omega$.

Teorema 1.2. Sejam $\Omega \subset \mathbb{R}^{n}$ um aberto conexo, $L$ um operador estritamente elíptico em $\Omega$ com coeficientes $a_{i j}=a_{j i} \in C^{1}(\Omega), b_{i}=c=0, i, j=1, \ldots, n$ e $u \in H^{2}(\Omega)$ uma função tal que

$$
|(L u)(x)| \leq K(|\nabla u(x)|+|u(x)|),
$$

em quase todo ponto de $\Omega$, para alguma constante $K>0$. Se $B$ é uma bola que intercepta a fronteira de $\Omega$ em uma hipersuperfície $B \cap \partial \Omega$ e $u=\frac{\partial u}{\partial N}=0$ em quase todo ponto de $B \cap \partial \Omega$, então $u=0$ em quase todo ponto de $\Omega$.

Teorema 1.3. Sejam $\Omega \subset \mathbb{R}^{n}$ um aberto de classe $C^{2}$ e $L$ um operador estritamente elíptico em $\Omega$, com coeficientes $a_{i j} \in C^{2}(\Omega), b_{i} \in C^{1}(\Omega), i, j=1, \ldots, n$ e $c \in L^{\infty}(\Omega)$. Sejam ainda $p, q>1$, $f \in L^{q}(\Omega)$ e suponha que $u \in L^{p}(\Omega)$ satisfaz

$$
\int_{\Omega} u L \varphi=\int_{\Omega} f \varphi
$$

para qualquer função $\varphi \in C^{2}(\Omega)$, com $\varphi=0$ sobre $\partial \Omega$. Então $u \in W^{2, q} \cap W_{0}^{1, q}(\Omega)$ e u é uma solução forte de

$$
\left\{\begin{array}{l}
L^{*} u=0 \text { em } \Omega \\
u=0 \text { em } \partial \Omega
\end{array}\right.
$$

Teorema 1.4. Sejam $\Omega \subset \mathbb{R}^{n}$ um dominio de classe $C^{1,1}(\Omega)$ e $L$ um operador estritamente elíptico com coeficientes $a_{i j} \in C^{0}(\Omega), b_{i}, c \in L^{\infty}(\Omega), i, j=1, \ldots, n$, com $c \leq 0$. Então, se $f \in L^{p}(\Omega)$ e $\varphi \in W^{2, p}(\Omega)$, com $1<p<\infty$, o problema de Dirichlet Lu $=f$ em $\Omega$ e $u-\varphi \in W_{0}^{1, p}(\Omega)$ tem uma única solução $u \in W^{2, p}(\Omega)$.

É consequência do teorema anterior o fato de que se $1<p<\infty$ e $L: W^{2, p} \cap W_{0}^{1, p}(\Omega) \longrightarrow$ $L^{p}(\Omega)$ é um operador estritamente elíptico com coeficientes $a_{i j} \in C^{0}(\Omega), b_{i}, c \in L^{\infty}(\Omega), i, j=$ $1, \ldots, n$, onde $c \leq 0$, então $L$ é um isomorfismo.

.Teorema 1.5. Seja $u \in W_{\text {loc }}^{2, p}(\Omega)$ uma solução da equação elíptica $L u=f$ em um domínio $\Omega$, em que os coeficientes de $L$ pertencem a $C^{k-1,1}(\Omega)\left(C^{k-1, \alpha}(\Omega)\right), f \in W_{\text {loc }}^{k, q}(\Omega)\left(C^{k-1, \alpha}(\Omega)\right)$, com $1<p, q<\infty, k \geq 1,0<\alpha<1$. Então $u \in W_{\text {loc }}^{k+2, q}(\Omega)\left(C^{k+1, \alpha}(\Omega)\right)$. Além disso, se $\Omega$ 
é de classe $C^{k+1,1}\left(C^{k+1, \alpha}\right)$ e L é estritamente elíptico em $\Omega$, com coeficientes em $C^{k-1,1}(\Omega)$ $\left(C^{k-1, \alpha}(\Omega)\right)$ e $f \in W^{k, q}(\Omega)\left(C^{k-1, \alpha}(\Omega)\right)$, então $u \in W^{k+2, q}(\Omega)$.

Sejam $\Omega \subset \mathbb{R}^{n}$ um domínio de classe $C^{3}$ e $f: \Omega \times \mathbb{R} \rightarrow \mathbb{R}$ uma função de classe $C^{2}$. Suponha que $u \in W^{2, p} \cap W_{0}^{1, p}(\Omega)$, com $p>n / 2$, seja solução $\Delta u+f(x, u)=0$ em $\Omega$. Como $W^{2, p}(\Omega) \subset C^{0, \alpha}(\Omega)$ para algum $0<\alpha<1$, vemos que $f(\cdot, u(\cdot)) \in C^{0, \alpha}(\Omega)$. Pelo Teorema 1.5, $u \in W^{3, q}(\Omega)$ para todo $q \in(0, \infty)$, resultando que $u \in C^{2, \nu}(\Omega)$ para todo $0<\nu<1$.

Sejam agora $\Omega \subset \mathbb{R}^{n}$ um domínio de classe $C^{3}, f: \mathbb{R}^{n} \times \mathbb{R} \times \mathbb{R}^{n} \rightarrow \mathbb{R}$ uma função de classe $C^{2}$ e $u \in W^{2, p} \cap W_{0}^{1, p}(\Omega), p>n$, solução de $\Delta u+f(x, u, \nabla u)=0$ em $\Omega$. Aqui, usando que $W^{2, p}(\Omega) \subset C^{1, \alpha}(\Omega)$ para algum $0<\alpha<1$, concluímos que $f(\cdot, u(\cdot), \nabla u(\cdot)) \in C^{0, \alpha}(\Omega)$. Novamente, usando o Teorema 1.5, obtemos que $u \in W^{3, q}(\Omega)$ para todo $q \in(0, \infty)$, do que decorre que $u \in C^{2, \nu}(\Omega)$ qualquer que seja $0<\nu<1$.

Os comentários acima se baseiam não somente no Teorema 1.5 como também no teorema sobre imersões de espaços de Sobolev que enunciamos abaixo.

Teorema 1.6. Seja $\Omega \subset \mathbb{R}^{n}$ um domínio Lipschitz regular. Temos as seguintes inclusões contínuas:

$$
\begin{aligned}
& W^{m, p}(\Omega) \subset W^{k, q}(\Omega) \text { se } m-\frac{n}{p} \geq k-\frac{n}{q}, p \leq q<\infty \\
& W^{m, p}(\Omega) \subset C^{\nu}(\Omega) \text { se } m-\frac{n}{p} \geq \nu \geq 0\left(\text { com } m-\frac{n}{p}>\nu\right. \text { se estes números são inteiros). }
\end{aligned}
$$

Se $\Omega$ é limitado, tais inclusões são compactas quando ocorre a desigualdade estrita: $m-n / p>$ $k-n / q$ ou $m-n / p>\nu$, respectivamente.

Encerramos este capítulo com a exposição de um resultado devido a Sard.

Teorema 1.7. Sejam $A \subset \mathbb{R}^{n}$ um aberto e $f: A \rightarrow \mathbb{R}^{m}$ uma aplicação de classe $C^{k}$, onde $k$ é um inteiro positivo e $k>n-m$. Então o conjunto dos valores críticos de $f$ tem medida nula em $\mathbb{R}^{m}$ e é magro. 


\section{Capítulo 2}

\section{Operadores de Fredholm}

Com vista à utilização do Teorema da Transversalidade enunciado por D. Henry, faremos um estudo prévio dos operadores de Fredholm limitados, em que obteremos propriedades que serão empregadas repetidas vezes nos próximos capítulos.

Sejam $X$ e $Y$ espaços de Banach. Dado um operador fechado $T: \mathcal{D}(T) \subset X \rightarrow Y$ tal que $\mathcal{R}(T)$ é subespaço fechado de $Y$, sejam $n(T)=\operatorname{dim} \mathcal{N}(T)$ e $d(T)=\operatorname{codim} \mathcal{R}(T)$. Chamamos $n(T)$ e $d(T)$ de nulidade e deficiência de $T$, respectivamente. Se ao menos uma delas, $n(T)$ ou $d(T)$, é finita, dizemos que $T$ é semi-Fredholm e definimos o índice de $T$ como sendo

$$
\text { ind } T=n(T)-d(T)
$$

Quando $\operatorname{ind} T<\infty$ (caso em que $\operatorname{dim} \mathcal{N}(T)<\infty$ ) $T$ é semi-Fredholm à esquerda e quando ind $T>-\infty$ (caso em que $\operatorname{codim} \mathcal{R}(T)<\infty) T$ é semi-Fredholm à direita. Se ambas, $d(T)$ e $n(T)$, são finitas (ou seja, se $T$ é semi-Fredholm à esquerda e à direita simultaneamente) $T$ é chamado um operador de Fredholm.

O resultado que apresentamos a seguir garante que se $T: \mathcal{D}(T) \subset X \rightarrow Y$ é um operador fechado é suficiente que tenhamos $\operatorname{codim} \mathcal{R}(T)<\infty$ para que $T$ seja semi-Fredholm à direita. Em particular, para concluírmos que $T$ é Fredholm, basta verificarmos que $\operatorname{dim} \mathcal{N}(T)<\infty$ e $\operatorname{codim} \mathcal{R}(T)<\infty$.

Proposição 2.1. Sejam $X$ e $Y$ espaços de Banach e $T: \mathcal{D}(T) \subset X \longrightarrow Y$ um operador linear fechado. Suponha que existe subespaço fechado $M$ de $Y$ tal que $\mathcal{R}(T) \cap M=\{0\}$ e o subespaço $\mathcal{R}(T) \oplus M$ é fechado. Então $\mathcal{R}(T)$ é fechado.

Demonstração. Seja $\mathcal{D}(T) \times M$ o domínio do operador linear $T_{1}: \mathcal{D}(T) \times M \subset X \times Y \rightarrow Y$ definido por

$$
T_{1}(x, m)=T x+m, \quad(x, m) \in \mathcal{D}(T) \times M .
$$

O fato de $T$ ser fechado nos leva facilmente à conclusão de que $T_{1}$ é também operador fechado. 
Como $\mathcal{R}\left(T_{1}\right)=\mathcal{R}(T) \oplus M$ é subsepaço fechado de $Y$, existe constante $c>0$ satisfazendo

$$
\left\|T_{1}(x, m)\right\|_{Y} \geq c \operatorname{dist}\left((x, m), \mathcal{N}\left(T_{1}\right)\right), \quad(x, m) \in \mathcal{D}(T) \times M .
$$

Em particular,

$$
\begin{aligned}
\|T x\|_{Y}=\left\|T_{1}(x, 0)\right\|_{X \times Y} & \geq c \operatorname{dist}\left((x, 0), \mathcal{N}\left(T_{1}\right)\right) \\
& =c \operatorname{dist}((x, 0), \mathcal{N}(T) \times\{0\}) \\
& =c \operatorname{dist}(x, \mathcal{N}(T)),
\end{aligned}
$$

resultando que $\mathcal{R}(T)$ é subsepaço fechado.

No que segue apresentamos alguns exemplos de operadores Fredholm e semi-Fredholm.

Exemplo 1. Se $X$ e $Y$ são espaços de dimensão finita, qualquer operador linear $T: X \rightarrow Y$ é Fredholm com ind $T=\operatorname{dim} X-\operatorname{dim} Y$. Com efeito, seja $n=\operatorname{dim} \mathcal{N}(T)$. Então $\operatorname{codim} \mathcal{R}(T)=$ $\operatorname{dim} Y-(\operatorname{dim} X-n)$. Logo, ind $T=n-(\operatorname{dim} Y-\operatorname{dim} X+n)=\operatorname{dim} X-\operatorname{dim} Y$ independe $\operatorname{de} T$.

Se um dos espaços $X, Y$ tem dimensão finita, situação análoga ocorre: todo operador $T \in$ $\mathcal{L}(X, Y)$ é semi-Fredholm e ind $T=\operatorname{dim} X-\operatorname{dim} Y$, como antes.

Exemplo 2. Se $X$ e $Y$ são espaços de Banach de dimensão infinita, operadores compactos de $X$ em $Y$ não são semi-Fredholm. De fato, se $K \in \mathcal{L}(X, Y)$ é um operador compacto com $\mathcal{R}(K)$ fechada, seja $Y_{1}=\mathcal{R}(K)$ e defina $K_{1} \in \mathcal{L}\left(X, Y_{1}\right)$ por $K_{1} x=K x, x \in X$. Da hipótese de que $\mathcal{R}(K)$ é fechada segue que $K_{1}$ é compacto e de $\mathcal{R}\left(K_{1}\right)=Y_{1}$ obtemos que o operador compacto $K_{1}^{*} \in \mathcal{L}\left(Y_{1}^{*}, X^{*}\right)$ possui inversa contínua. Então $I_{Y_{1}^{*}}=\left(K_{1}^{*}\right)^{-1} K_{1}^{*}$ é compacta, implicando que $\operatorname{dim} Y_{1}^{*}<\infty$. Logo, $\operatorname{dim} Y_{1}<\infty$. Quanto à dimensão do núcleo de $K$, observamos que não podemos ter $\operatorname{dim} \mathcal{N}(K)<\infty$ pois nesse caso construíriamos um isomorfismo entre um espaço de dimensão infinita e outro de dimensão finita.

Exemplo 3. Considere $l_{2}$ o espaço das sequências $x=\left(x_{1}, x_{2}, \ldots\right)$ de números reais tais que $\sum_{n=1}^{\infty} x_{n}^{2}<\infty$

A translação $S:\left(x_{1}, x_{2}, x_{3}, \ldots\right) \longmapsto\left(x_{2}, x_{3}, x_{4}, \ldots\right)$ é Fredholm com índice 1 e, em geral, $S^{m}$ é Fredholm com índice $m$, para $m=1,2, \ldots$ A adjunta de $S, S^{*}:\left(x_{1}, x_{2}, x_{3}, \ldots\right) \longmapsto$ $\left(0, x_{1}, x_{2}, x_{3}, \ldots\right)$, é Fredholm com índice -1 e $\left(S^{*}\right)^{m}$ tem índice $-m, m=1,2, \ldots$

O operador $L:\left(x_{1}, x_{2}, x_{3}, \ldots\right) \longmapsto\left(x_{1}, 0, x_{2}, 0, \ldots\right)$ é semi-Fredholm à esquerda, com índice $-\infty$ e seu adjunto $L^{*}:\left(x_{1}, x_{2}, x_{3}, \ldots\right) \longmapsto\left(x_{1}, x_{3}, x_{5}, \ldots\right)$ é semi-Fredholm à direita com índice $\infty$. Observe que $L^{*} L$ é a identidade, mas $L L^{*}$ não é semi-Fredholm.

Os dois próximos teoremas evidenciam a conexão entre os operadores de Fredholm e os operadores da forma $I+K$, em que $K \in \mathcal{L}(X)$ é um operador compacto: os operadores de Fredholm são, na verdade, uma generalização dos operadores da forma $I+K$ em que $K$ é compacto. 
Teorema 2.2. Sejam $X$ um espaço de Banach e $K \in \mathcal{L}(X)$ um operador compacto. Então

(i) $\mathcal{N}(I-K)$ tem dimensão finito.

(ii) $\mathcal{R}(I-K)=\mathcal{N}\left(I-K^{*}\right)^{\perp}$ (portanto, $\mathcal{R}(I-K)$ tem codimensão finita).

(iii) $\mathcal{N}(I-K)=\{0\}$ se, e somente se, $\mathcal{R}(I-K)=X$.

Demonstração. (i) Se $B$ é a bola unitária fechada em $\mathcal{N}(I-K)$, então $B=K(B)$. Sendo $K$ compacto, $B$ é compacto, o que garante que $\mathcal{N}(I-T)$ tem dimensão finita.

(ii) Como $\operatorname{dim} \mathcal{N}(I-K)<\infty$, existe subsepaço fechado $X_{1} \subset X$ tal que $X=\mathcal{N}(I-K) \oplus X_{1}$. Seja $S \in \mathcal{L}\left(X_{1}, X\right)$ definido por $S x=x-K x, x \in X_{1}$. Claramente, $S$ é injetora e $\mathcal{R}(S)=\mathcal{R}(I-K)$. É conhecido o fato de que $\mathcal{N}\left(I-K^{*}\right)=\mathcal{R}(I-K)^{\perp}$ (ver [5]) e, portanto, $\mathcal{N}\left(I-K^{*}\right)^{\perp}=$ $\left(\mathcal{R}(I-K)^{\perp}\right)^{\perp}=\overline{\mathcal{R}(I-K)}$. Basta então verificarmos que $\mathcal{R}(I-K)$ é subespaço fechado de $X$, o que faremos provando que existe $r>0$ tal que $\|S x\| \geq r\|x\|$ para todo $x \in X_{1}$.

Suponha, por absurdo, que não exista um tal $r>0$. Então existe uma sequência $\left\{x_{n}\right\}$ de vetores unitários em $X_{1}$ tal que $S x_{n} \longrightarrow 0$. De fato, fazendo $r_{n}=1 / n, n \in \mathbb{N}$, concluímos que existe uma sequência $\left\{\bar{x}_{n}\right\}$ em $X_{1}$ com a propriedade de que

$$
\left\|S \tilde{x}_{n}\right\|<\frac{\left\|\bar{x}_{n}\right\|}{n}, \quad n \in \mathbb{N} .
$$

Tomando $x_{n}=\tilde{x}_{n} /\left\|\tilde{x}_{n}\right\|, n \in \mathbb{N}$, obtemos a sequência desejada.

Pela compacidade de $K$, existe uma subsequência $\left\{x_{n_{k}}\right\}$ tal que $K x_{n_{k}} \longrightarrow x_{0}$. Assim, $x_{n_{k}}=S x_{n_{k}}+K x_{n_{k}} \longrightarrow x_{0}$, com $\left\|x_{0}\right\|=1$. Logo, $S x_{0}=\lim S x_{n_{k}}=0$, contrariando o fato de que $\mathcal{N}(S)=\{0\}$.

(iii) Suponha que $\mathcal{N}(I-T)=\{0\}$ e assuma, para obter uma contradição, que $X_{1}=\mathcal{R}(I-K) \nsubseteq$ $X$. Por (ii), sabemos que $X_{1}$ é subespaço fechado de $X$, de maneira que $X_{1}$, com a norma herdada de $X$, é um espaço de Banach. Notamos também que

$$
K\left(X_{1}\right)=K((I-K)(X))=(I-K) K(X) \subset(I-K)(X)=X_{1},
$$

do que segue que $\left.K\right|_{X_{1}}: X_{1} \longrightarrow X_{1}$ é também um operador compacto. Seja $X_{2}=(I-K)\left(X_{1}\right)$. Novamente, por (ii), $X_{2}$ é subespaço fechado de $X_{1}$. Além disso, $X_{2} \nsubseteq X_{1}$. Com efeito, escolha $x \in X \backslash X_{1}$ e vejamos que $(I-K) x \notin X_{2}$. Se $(I-K) x \in X_{2}$, teríamos $(I-K) x=(I-K) x_{1}$ para algum $x_{1} \in X_{1}$. Como $\mathcal{N}(I-K)=\{0\}$, concluiríamos que $x \in X_{1}$, contrariando a escolha de $x$.

Em geral, tomando $X_{n}=(I-K)^{n}(X)$ obtemos uma sequência estritamente decrescente de subespaços fechados de $X$. O Lema de Riesz (ver [5]) garante a existência de uma sequência $\left\{u_{n}\right\}$ satisfazendo $\left\|u_{n}\right\|=1, u_{n} \in X_{n} \backslash X_{n+1}$ e $\operatorname{dist}\left(u_{n}, X_{n+1}\right) \geq 1 / 2$, para cada $n \in \mathbb{N}$.

Se $n>m$, considerando que

$$
X_{n+1} \subset X_{n} \subset X_{m+1} \subset X_{m}
$$


vemos que

$$
-(I-K) u_{n}+(I-K) u_{m}+u_{n} \in X_{m+1} .
$$

Desse modo,

$$
\begin{aligned}
\left\|K u_{n}-K u_{m}\right\| & =\left\|\left[-(I-K) u_{n}+(I-K) u_{m}+u_{n}\right]-u_{m}\right\| \\
& \geq \operatorname{dist}\left(u_{m}, X_{m+1}\right) \geq \frac{1}{2},
\end{aligned}
$$

o que implica que $\left\{K u_{n}\right\}$ não admite subsequência convergente, contrariando a compacidade de $K$. Segue que $\mathcal{R}(I-K)=X$.

Reciprocamente, suponha que $\mathcal{R}(I-K)=X$. Neste caso, $\mathcal{N}\left(I-K^{*}\right)=\mathcal{R}(I-K)^{\perp}=X^{\perp}=$ $\{0\}$. Sendo $K^{*}$ compacto, $\mathcal{R}\left(I-K^{*}\right)^{\perp}=X^{*}$. Por fim, $\mathcal{N}(I-K)=\mathcal{R}\left(I-K^{*}\right)^{\perp}=\left(X^{*}\right)^{\perp}=$ $\{0\}$.

Teorema 2.3. Sejam $X$ um espaço de Banach e $K \in \mathcal{L}(X)$ um operador compacto. Entāo $I-K$ é um operador de Fredholm com índice zero.

Demonstração. Sejam $n=\operatorname{dim} \mathcal{N}(I-K)$ e $r=\operatorname{codim} \mathcal{R}(I-K)$. Assuma que $n>r$. Temos que $X=\mathcal{N}(I-K) \oplus X_{1}$ e $X=\mathcal{R}(I-K) \oplus X_{2}$, para subespaços fechados $X_{1}$ e $X_{2}$, com $\operatorname{dim} X_{2}=r$.

Como $n>r$, existe transformação linear $A: \mathcal{N}(I-K) \longrightarrow X_{2}$ sobrejetora, com núcleo não trivial. Defina $\vec{K}=K-A P$, onde $P \in \mathcal{L}(X)$ é a projeção de $X$ sobre $\mathcal{N}(I-K)$ ao longo de $X_{\mathrm{I}}$. Note que $A P \in \mathcal{L}(X)$ é um operador compacto pois $\operatorname{dim} \mathcal{R}(A P)<\infty$.

Se $x \in X_{1}$ então $P x=0$ e $\bar{K} x=K x$, o que fornece $(I-\bar{K})\left(X_{1}\right)=(I-K)\left(X_{1}\right)=$ $\mathcal{R}(I-K)$. Ainda, se $x \in \mathcal{N}(I-K)$ então $(I-\bar{K}) x=(x-K x)+A x=A x$, mostrando que $(I-\bar{K})(\mathcal{N}(I-K))=A(\mathcal{N}(I-K))=X_{2}$. Logo, fica evidente que $X=\mathcal{R}(I-K) \oplus X_{2} \subset \mathcal{R}(I-\bar{K})$, isto é, $\mathcal{R}(I-\bar{K})=X$.

Agora, existe $x_{0} \in \mathcal{N}(I-T) \backslash\{0\}$ tal que $A x_{0}=0$, resultando que $\bar{K} x_{0}-x_{0}=K x_{0}+A x_{0}-$ $x_{0}=K x_{0}-x_{0}=0$. Então $\bar{K}$ é um operador compacto com $\mathcal{N}(I-\bar{K}) \neq\{0\}$ e $\mathcal{R}(I-\bar{K})=X$, contrariando a conclusão do Teorema 2.2. Portanto, $n \leq r$.

Observe que $\operatorname{dim} \mathcal{N}\left(I-K^{*}\right)=\operatorname{codim} \mathcal{R}(I-K)=r \mathrm{e} \operatorname{codim} \mathcal{R}\left(I-K^{*}\right)=\operatorname{dim} \mathcal{N}(I-K)=n$ e podemos repetir o mesmo argumento com $K^{*}$ em lugar de $K$ para verificar que $n \geq r$.

A utilização do Teorema 2.2 permite a obtenção de uma caracterização dos operadores de Fredholm limitados, enunciada a seguir.

Teorema 2.4. Sejam $X$ e $Y$ espaços de Banach e $T \in \mathcal{L}(X, Y)$. As seguintes afirmações são equivalentes:

(i) T é Fredholm. 
(ii) Existe $S \in \mathcal{L}(Y, X)$ tal que $T S-I \in \mathcal{L}(Y)$ e $S T-I \in \mathcal{L}(X)$ são operadores compactos.

(iii) Existem $S_{1}, S_{2} \in \mathcal{L}(X, Y)$ tais que $T S_{1}-I \in \mathcal{L}(Y)$ e $S_{2} T-I \in \mathcal{L}(X)$ são operadores compactos.

Demonstração. A implicação (ii) $\Rightarrow$ (iii) é imediata. Suponha que existam $S_{1}, S_{2} \in \mathcal{L}(Y, X)$ tais que $K_{1}=T S_{1}-I$ e $K_{2}=S_{2} T-I$ são operadores compactos. Temos

$$
\begin{aligned}
& \mathcal{N}(T) \subset \mathcal{N}\left(S_{2} T\right)=\mathcal{N}\left(I+K_{2}\right) \\
& \mathcal{R}(T) \supset \mathcal{R}\left(T S_{1}\right)=\mathcal{R}\left(I+K_{1}\right)
\end{aligned}
$$

Como $\operatorname{dim} \mathcal{N}\left(I+K_{2}\right)<\infty$ e $\operatorname{codim} \mathcal{R}\left(I+K_{1}\right)<\infty$, segue que $\operatorname{dim} \mathcal{N}(T)<\infty$ e $\operatorname{codim} \mathcal{R}(T)<$ $\infty$.

Admita agora que $T$ é Fredholm. Então exsitem subespaços fechados $E \subset X$ e $F \subset Y$ tais que $X=\mathcal{N}(T) \oplus E$ e $Y=F \oplus \mathcal{R}(T)$. Seja $T_{0}: E \longrightarrow \mathcal{R}(T)$ o operador dado por $T_{0} x=T x$, $x \in E . T_{0}$ é claramente um isomorfismo. Defina $S: Y \longrightarrow X$ por

$$
S(f+T e)=e, \quad e \in E, f \in F .
$$

Se $P: Y \longrightarrow Y$ é a projeção (contínua) de $Y$ sobre $\mathcal{R}(T)$ ao longo de $F$, temos

$$
\begin{aligned}
\|S(f+T e)\|_{X} & =\|S(P(f+T e))\|_{X}=\left\|T_{0}^{-1}(P(f+T e))\right\|_{X} \\
& \leq\left\|T_{0}^{-1}\right\|_{\mathcal{L}(\mathcal{R}(T), E)}\|P\|_{\mathcal{L}(Y)}\|f+T e\|_{Y},
\end{aligned}
$$

para quaisquer $e \in E, f \in F$, o que demonstra que $S \in \mathcal{L}(Y, X)$.

Resta ainda verificar que $S T-I$ e $T S-I$ são operadores compactos. Sejam $Q_{1}: X \longrightarrow X$ a projeção de $X$ sobre $\mathcal{N}(T)$ ao longo de $E$ e $Q_{2}: Y \longrightarrow Y$ a projeção de $Y$ sobre $F$ ao longo de $\mathcal{R}(T)$. Tais projeções são compactas uma vez que $\mathcal{N}(T)$ e $F$ têm dimensão finita.

Se $x \in X$, podemos escrever $x=u+e, \operatorname{com} u \in \mathcal{N}(T)$ e $e \in E$. Assim,

$$
(S T-I) x=S T(u+e)-(u+e)=S T e-(u+e)=-u
$$

provando que $S T-I=-Q_{1}$ é um operador compacto. Da mesma forma, podemos escrever $y \in Y$ como $y=f+T e$, onde $f \in F$ e $e \in E$. Neste caso, temos

$$
(T S-I) y=(T S-I)(f+T e)=T S(T e)-(f+T e)=-f
$$

o que estabelece que $T S-I=-Q_{2}$ é também um operador compacto.

A propriedade de um operador $T \in \mathcal{L}(X, Y)$ ser semi-Fredholm se transfere para o seu conjugado $T^{*} \in \mathcal{L}\left(Y^{*}, X^{*}\right)$. Embora os índices de $T$ c $T^{*}$ não coincidam, eles guardam uma forte relação, expressa no teorema abaixo. 
Teorema 2.5. Sejam $X$ e $Y$ espaços de Banach e $T \in \mathcal{L}(X, Y)$. T é semi-Fredholm se, e somente se $T^{*}$ é semi-Fredholm. Neste caso, ind $T^{*}=-$ ind $T$.

Demonstração. O Teorema 5.13 em [14] afirma que $\mathcal{R}(T)$ é fechada se, e somente se, $\mathcal{R}\left(T^{*}\right)$ é fechada. Logo, $\mathcal{R}(T)=\mathcal{N}\left(T^{*}\right)$ o que fornece $\operatorname{codim} \mathcal{R}(T)=\operatorname{dim} \mathcal{N}(T)^{\perp}$ e $\mathcal{R}\left(T^{*}\right)=\mathcal{N}(T)^{\perp}$, implicando $\operatorname{codim} \mathcal{R}\left(T^{*}\right)=\operatorname{dim} \mathcal{N}(T)$.

O subconjunto de $\mathcal{L}(X, Y)$ formado pelos operadores semi-Fredholm é aberto em $\mathcal{L}(X, Y)$ conforme expressa o enunciado do próximo teorema.

Teorema 2.6. Sejam $X$ e $Y$ espaços de Banach. Se $T \in \mathcal{L}(X, Y)$ é semi-Fredholm, existe $\delta>0$ tal que se $\|S-T\|_{\mathcal{L}(X, Y)}<\delta$ então $S$ é semi-Fredholm, com ind $S=\operatorname{ind} T$.

Demonstração. Vamos supor inicialmente que $T$ é Fredholm. Então $X_{1}=\mathcal{N}(T)$ e $Y_{2}=\mathcal{R}(T)$ são subespaços fechados de $X$ e $Y$, respectivamente. Além disso, existem subsepaços fechados $X_{2} \subset X$ e $Y_{1} \subset Y$ (este último de dimensão finita) tais que $X=X_{1} \oplus X_{2}$ e $Y=Y_{1} \oplus Y_{2}$.

Considerando tais decomposições dos espaços $X$ e $Y$, cada operador $A \in \mathcal{L}(X, Y)$ admite uma representação matricial na forma

$$
A=\left(\begin{array}{ll}
A_{11} & A_{12} \\
A_{21} & A_{22}
\end{array}\right):\left(\begin{array}{c}
X_{1} \\
X_{2}
\end{array}\right) \longrightarrow\left(\begin{array}{c}
Y_{1} \\
Y_{2}
\end{array}\right):\left(\begin{array}{c}
x_{1} \\
x_{2}
\end{array}\right) \longmapsto\left(\begin{array}{c}
A_{11} x_{1}+A_{12} x_{2} \\
A_{21} x_{1}+A_{22} x_{2}
\end{array}\right)
$$

$\operatorname{com} A_{i j} \in \mathcal{L}\left(X_{i}, Y_{j}\right), i, j=1,2$.

Em particular,

$$
T=\left(\begin{array}{ll}
T_{11} & T_{12} \\
T_{21} & T_{22}
\end{array}\right)
$$

mas $T_{11}=0$ e $T_{12}=0$ já que $\mathcal{R}(T)=Y_{2}$ e $T_{21}=0$ pois $\mathcal{N}(T)=X_{1}$, de modo que, na realidade,

$$
T=\left(\begin{array}{cc}
0 & 0 \\
0 & T_{22}
\end{array}\right)
$$

onde $T_{22}: X_{2} \longrightarrow Y_{2}$ é claramente um isomorfismo.

Seja $L: Y \longrightarrow Y$ a projeção de $Y$ sobre $Y_{2}$ paralelamente a $Y_{1}$. Para cada $A \in \mathcal{L}(X, Y)$ temos

$$
\left\|A_{22}-T_{22}\right\|_{\mathcal{L}\left(X_{2}, Y_{2}\right)} \leq\|L\|_{\mathcal{L}(Y)}\|A-T\|_{\mathcal{L}(X, Y)}
$$

Sendo o subconjunto dos operadores inversíveis de $\mathcal{L}\left(X_{2}, Y_{2}\right)$ um subconjunto aberto, podemos escolher $\delta>0$ tal que $S \in \mathcal{L}(X, Y)$ e $\|S-T\|_{\mathcal{L}(X, Y)}<\delta$ implicam que $S_{22}$ é um isomorfismo.

Fixado $S \in \mathcal{L}(X, Y)$ satisfazendo $\|S-T\|_{\mathcal{L}(X, Y)}<\delta$, tome 
onde

$$
\begin{aligned}
& P=-S_{12} S_{22}^{-1} \in \mathcal{L}\left(Y_{2}, Y_{1}\right) \\
& Q=-S_{22}^{-1} S_{21} \in \mathcal{L}\left(X_{1}, X_{2}\right)
\end{aligned}
$$

Note que

$$
\left(\begin{array}{cc}
I_{Y_{1}} & P \\
0 & i_{y_{2}}
\end{array}\right)^{-1}=\left(\begin{array}{cc}
I_{Y_{1}} & -P \\
0 & I_{Y_{2}}
\end{array}\right) \text { e }\left(\begin{array}{cc}
I_{X_{1}} & 0 \\
Q & I_{X_{2}}
\end{array}\right)^{-1}=\left(\begin{array}{cc}
I_{X_{1}} & 0 \\
-Q & I_{X_{2}}
\end{array}\right)
$$

e, portanto,

$$
\left(\begin{array}{cc}
I_{Y_{1}} & P \\
0 & I_{y_{2}}
\end{array}\right) \text { e }\left(\begin{array}{cc}
I_{X_{1}} & 0 \\
Q & I_{X_{2}}
\end{array}\right)
$$

são isormorfismos de $Y$ em $Y$ e de $X$ em $X$, respectivamente.

Por fim, observando que

$$
\tilde{S}=\left(\begin{array}{cc}
S_{11}-S_{12} S_{22}^{-1} S_{21} & 0 \\
0 & S_{22}
\end{array}\right)
$$

chegamos à conclusão de que $\operatorname{codim} \mathcal{R}(\tilde{S})=\operatorname{codim} \mathcal{R}\left(S_{11}-S_{12} S_{22}^{-1} S_{21}\right)$ e $\operatorname{dim} \mathcal{N}(\tilde{S})=\operatorname{dim} \mathcal{N}\left(S_{11}-\right.$ $\left.S_{12} S_{22}^{-1} S_{21}\right)$, onde $S_{11}-S_{12} S_{22}^{-1} S_{21} \in \mathcal{L}\left(X_{1}, Y_{1}\right)$. Como $X_{1}$ e $Y_{1}$ são espaços de dimensão finita, obtemos que

$$
\text { ind } S=\text { ind } \tilde{S}=\operatorname{dim} X_{1}-\operatorname{dim} Y_{1}=\operatorname{ind} T \text {. }
$$

Tratemos agora o caso em que $T$ é semi-Fredholm à esquerda, isto é, $\mathcal{N}(T)$ tem dimensão finita e $\mathcal{R}(T)$ é fechada. Se $X_{1}=\mathcal{N}(T)$, podemos escolher subespaço fechado $X_{2}$ tal que $X=X_{1} \oplus X_{2}$. Pelo Teorema do Gráfico Fechado, a restrição de $T$ a uma aplicaçāo de $X_{2}$ em $\mathcal{R}(T)$ é um isomorfismo. Logo, existe $c>0$ satisfazendo

$$
\|T x\| \geq c\|x\|
$$

para todo $x \in X_{2}$. Se $S \in \mathcal{L}(X, Y)$ com $\|S-T\| \leq \frac{c}{8}$, para $x \in X_{2}$

$$
\|S x\| \geq\|T x\|-\|S-T\|\|x\| \geq \frac{7 c}{8}\|x\|
$$

de maneira que a restrição de $S$ a $X_{2}$ é injetora e tem imagem fechada. Como $X_{1}$ tem dimensāo finita, $\mathcal{R}(S)=S\left(X_{2}\right)+S\left(X_{1}\right)$ é fechada. Além disso, $\operatorname{dim} \mathcal{N}(S) \leq \operatorname{dim} X_{1}$. Portanto $S$ é semiFredholm à esquerda.

Admita que ind $T=-\infty$, ou seja, $\mathcal{R}(T)$ tem codimensão infinita. Usando o Lema de Riesz podemos então construir uma sequência $\left\{y_{n}\right\}$ em $Y$ tal que

$$
\left\|y_{n}\right\|=1 \text { e } \operatorname{dist}\left(y_{n},\left[\mathcal{R}(T) \cup\left\{y_{1}, \ldots, y_{n-1}\right\}\right) \geq \frac{3}{4},\right.
$$

qualquer que seja $n \in \mathbb{N}$. 
Assim, se $j>k$ e $x \in X_{2}$, temos

$$
\begin{aligned}
\left\|y_{j}-y_{k}-S x\right\| & \geq \max \left\{\left\|y_{j}-y_{k}-T x\right\|-\frac{c}{8}\|x\|_{2}\|S x\|-2\right\} \\
& \geq \max \left\{\frac{3}{4}-\frac{c}{8}\|x\|, \frac{7 c}{8}\|x\|-2\right\} \\
& \geq \frac{7}{8}\left(\frac{3}{4}-\frac{c}{8}\|x\|\right)+\frac{1}{8}\left(\frac{7 c}{8}\|x\|-2\right)=\frac{13}{32} .
\end{aligned}
$$

Isto implica que $S\left(X_{2}\right)$ tem codimensão infinita. Com efeito, se isto não for verdade, escolha um subespaço de dimensão finita $Y_{1}$ tal que $Y=Y_{1} \oplus S\left(X_{2}\right)$ e seja $P \in \mathcal{L}(Y)$ a projeção sobre $Y_{1}$ ao longo de $S\left(X_{2}\right)$.

Para cada $n \in \mathbb{N}$ existe $x_{n} \in X_{2}$ tal que $y_{n}=P y_{n}+S x_{n}$ e se $j>k$ teremos

$$
\left\|P y_{j}-P y_{k}\right\|=\left\|y_{j}-y_{k}-S\left(x_{j}-x_{k}\right)\right\| \geq \frac{13}{32},
$$

resultando que $\left\{P y_{n}\right\}$ é um sequência limitada em $\mathcal{R}(P)$ que não admite subsequências convergentes, o que contraria o fato de $\mathcal{R}(P)$ ter dimensāo finita.

Segue que $\operatorname{codim} S\left(X_{2}\right)=\infty$ e, por conseguinte, $\operatorname{codim} R(S)=\infty$, uma vez que $\mathcal{R}(S)=$ $S\left(X_{1}\right)+S\left(X_{2}\right)$ e $S\left(X_{1}\right)$ tem dimensão finita.

$O$ caso que ainda não tratamos, ou seja, aquele em que $T$ é semi-Fredholm à direita, pode ser reduzido ao anterior se levamos em consideração o Teorema 2.5 e o fato de que $T_{1}^{*}$ se aproxima arbitrariamente de $T^{*}$ quando $T_{1}$ se aproxima suficientemente de $T$.

Corolário 2.7. Sejam $X$ e $Y$ espaços de Banach e $T \in \mathcal{L}(X, Y)$ um operador semi-Fredholm sobrejetor. Então todo $S$ suficientemente próximo de $T$ é também sobrejetor.

Demonstração. Para $S$ próximo de $T$ temos ind $S=\operatorname{ind} T=\operatorname{dim} \mathcal{N}(T)$ e $\operatorname{dim} \mathcal{N}(S) \leq$ $\operatorname{dim} \mathcal{N}(T)$, como está evidente na demonstração anterior. Portanto,

$$
\operatorname{codim} \mathcal{R}(S)=\operatorname{dim} \mathcal{N}(S)-\operatorname{ind} S \leq \operatorname{dim} \mathcal{N}(T)-\operatorname{ind} T=\operatorname{codim} \mathcal{R}(T)=0,
$$

provando o resultado.

Além de mostrar que o conjunto dos operadores semi-Fredholm é um aberto de $\mathcal{L}(X, Y)$, o Teorema 2.6 fornece ainda que o índice é constante nas componentes conexas daquele conjunto.

Utilizando o Teorema 2.4 conseguimos demonstrar de modo muito simples que dados operadores $T, K \in \mathcal{L}(X, Y)$, com $T$ Fredholm e $K$ compacto, a soma $T+K$ é Fredholm e, além disso, ind $(T+K)=$ ind $T$. Esta afirmação pode ser estendida para operadores semi-Fredholm, mas exige uma demonstração mais cuidadosa.

Teorema 2.8. Sejam $X$ e $Y$ espaços de Banach e $T \in \mathcal{L}(X, Y)$ um operador semi-Fredhom. Se $K \in \mathcal{L}(X, Y)$ é compacto então $T+K$ é semi-Fredholm, com ind $(T+K)=\operatorname{ind} T$. 
Demonstração. Suponha inicialmente que $T$ é semi-Fredholm à esquerda. Se $X_{1}=\mathcal{N}(T)$, escolha um complementar fechado $X_{2}$ para $X_{1}$.

Seja $\left\{x_{n}\right\}$ uma sequência limitada em $\mathcal{N}(T+K)$. Escrevendo $x_{n}=x_{n}^{1}+x_{n}^{2}, x_{n}^{1} \in X_{1}$, $x_{n}^{2} \in X_{2}$, notamos que $\left\{x_{n}^{1}\right\}$ e $\left\{K x_{n}\right\}$ estão em conjuntos compactos, o que permite a escolha de uma subsequência $\left\{x_{n_{j}}\right\}$ tal que as sequências $\left\{x_{n_{j}}^{1}\right\}$ e $\left\{K x_{n_{j}}\right\}$ convergem. Logo, $\left\{T x_{n_{j}}\right\}$ e $\left\{T x_{n_{j}}^{1}\right\}$ são também convergentes, do que decorre a convergência de $\left\{T x_{n_{j}}^{2}\right\}$. Observando que a restrição de $T$ a uma transformação linear entre os espaços $X_{2}$ e $\mathcal{R}(T)$ é um isomorfismo (pelo Teorema do Gráfico Fechado), concluímos que $\left\{x_{n_{j}}\right\}$ converge. Com isso provamos que conjunto limitado em $\mathcal{N}(T+K)$ é relativamente compacto, o que implica $\operatorname{dim} \mathcal{N}(T+K)<\infty$.

Como $\operatorname{dim} \mathcal{N}(T+K)<\infty$, podemos escrever $X_{2}=\left(X_{2} \cap \mathcal{N}(T+K)\right) \oplus X_{3}$ para algum subespaço fechado $X_{3}$, de modo que $\mathcal{R}(T+K)=K\left(X_{1}\right)+(T+K)\left(X_{3}\right)$. Isto mostra que $\mathcal{R}(T+K)$ será fechada se, e somente se, $(T+K)\left(X_{3}\right)$ for fechado, conclusão a que chegamos baseados no fato de que $\operatorname{dim} X_{1}<\infty$.

Para verificarmos que $(T+K)\left(X_{3}\right)$ é fechado, seja $\left\{x_{n}\right\}$ sequência em $X_{3}$ tal que $(T+$ $K) x_{n} \longrightarrow y$. Suponha, por absurdo, que $\left\{x_{n}\right\}$ não seja limitada. Podemos admitir que $\left\|x_{n}\right\| \longrightarrow$ $\infty$ (senão, passamos a uma subsequência com esta propriedade). Neste caso, $u_{n}=x_{n} /\left\|x_{n}\right\|$ é uma sequência de vetores unitários em $X_{3}$ e $(T+K) u_{n} \rightarrow 0$. Sendo $K$ compacto, existe uma subsequência $\left\{u_{n_{j}}\right\}$ tal que $\left\{K u_{n_{j}}\right\}$ converge e, conseqüentemente, $\left\{T u_{n_{j}}\right\}$ converge. Novamente, por ser a restrição de $T$ a uma transformação linear entre os espaços $X_{2}$ e $\mathcal{R}(T)$ um isomorfismo, concluímos que $\left\{u_{n_{j}}\right\}$ converge, digamos, para $u \in X_{3}$. Mas então $(T+K) u=0$, com $u \in X_{3} \mathrm{e}$ $\|u\|=1$, uma contradição.

Portanto, $\left\{x_{n}\right\}$ é limitada e utilizando uma vez mais a hipótese de que $K$ é compacto é possivel supor que $\left\{K x_{n}\right\}$ converge, do que segue a convergência de $\left\{T x_{n}\right\}$ que, por sua vez, fornece a convergência de $\left\{x_{n}\right\}$. Assim, $x_{n} \longrightarrow x \in X_{3}$ e $y=(T+K) x \in(T+K)\left(X_{3}\right)$.

Até aqui provamos apenas que $T+K$ é semi-Fredholm à esquerda, sem obter informação sobre o seu índice. Para obtê-la, observamos que $T+t K$ é semi-Fredholm à esquerda para todo $t \in[0,1]$ e, pelo Teorema $2.6, t \longmapsto$ ind $(T+t K)$ é localmente constante e, por conseguinte, constante. Logo, ind $(T+K)=\operatorname{ind} T$.

Finalmente, se $T$ não é semi-Fredholm à esquerda então o Teorema 2.5 e o fato de $K^{*}$ ser compacto possibilitam a redução do problema ao caso anterior, completando a demonstração. 
Exemplo 4. Seja $\Omega \subset \mathbb{R}^{n}$ um conjunto aberto, conexo e limitado, com fronteira $C^{2}$-regular. Considere $L$ o operador linear

$$
\begin{aligned}
& L: W^{2, p} \cap W_{0}^{1, p}(\Omega) \longrightarrow L^{p}(\Omega) \\
& u \longmapsto \sum_{i, j=1}^{n} a_{i j}(x) \frac{\partial^{2} u}{\partial x_{j} \partial x_{i}}+\sum_{i=1}^{n} b_{i}(x) \frac{\partial u}{\partial x_{i}}+c(x) u,
\end{aligned}
$$

com coeficientes $a_{i j} \in C^{0}(\Omega), b_{i}, c \in L^{\infty}(\Omega)$, para $i, j=1, \ldots, n$. Suponha que $L$ é um operador estritamente elíptico em $\Omega$, ou seja, existe uma constante $\alpha>0$ tal que

$$
\sum_{i, j=1}^{n} a_{i j}(x) \xi_{i} \xi_{j} \geq \alpha|\xi|^{2}, x \in \Omega, \xi=\left(\xi_{1}, \ldots, \xi_{n}\right) \in \mathbb{R}^{n} \backslash\{0\}
$$

Pelo Teorema 1.4, se tomarmos $\lambda>\|c\|_{L^{\infty}(\Omega)}$ então $L-\lambda: W^{2, p} \cap W_{0}^{1, p}(\Omega) \longrightarrow L^{p}(\Omega)$ é um isomorfismo e, portanto, um operador de Fredholm com índice zero. Como $W^{2, p}(\Omega) \hookrightarrow L^{p}(\Omega)$ (usamos o símbolo $\hookrightarrow$ para indicar imersão compacta), o operador $\lambda: W^{2, p} \cap W_{0}^{1, p}(\Omega) \longrightarrow L^{p}(\Omega)$ é compacto e pelo Teorema 2.8 segue que $L=(L-\lambda)+\lambda$ é um operador de Fredholm com índice zero.

Exemplo 5. Sejam $\Omega \subset \mathbb{R}^{n}$ um aberto limitado com fronteira $C^{3}$-regular, $p>n / 2$ e $L: W^{2, p} \cap W_{0}^{1, p}(\Omega) \longrightarrow L^{p}(\Omega)$ o operador dado por $L u=\Delta u+a(x) u$, onde $a \in L^{\infty}(\Omega)$. Então

$$
\mathcal{R}(L)=\left\{v \in L^{p}(\Omega) ; \int_{\Omega} u v=0 \text { para toda } u \in \mathcal{N}(L)\right\} .
$$

De fato, seja $\left\{u_{1}, \ldots, u_{n}\right\}$ uma base de $\mathcal{N}(L)$. Sabemos que $u_{i} \in C^{2, \alpha}(\Omega), i=1, \ldots, n$, para todo $\alpha \in(0,1)$. A limitação de $\Omega$ assegura que $u_{i} \in L^{q}(\Omega)$, onde $q>1$ e $p^{-1}+q^{-1}=1$. Observando que

$$
\int_{\Omega} u_{i} L \dot{u}=\int_{\Omega}(\Delta \dot{u}+a \dot{u}) u_{i}=\int_{\Omega}\left(\Delta u_{i}+a u_{i}\right) \dot{u}=0, \quad \dot{u} \in W^{2, p} \cap W_{0}^{1, p}(\Omega),
$$

para $i=1, \ldots, n$, concluímos que $\left\{u_{1}, \ldots, u_{n}\right\} \subset \mathcal{N}\left(L^{*}\right)$. Segue do exemplo anterior que $\left\{u_{1}, \ldots, u_{n}\right\}$ é base de $\mathcal{N}\left(L^{*}\right)$ e $\mathcal{R}(L)=\overline{\mathcal{R}(L)}=\mathcal{N}\left(L^{*}\right)^{\perp}$, o que prova nossa afirmação.

A composição de dois operadores semi-Fredholm limitados produz, desde que seus índices possam ser somados, um operador semi-Fredholm limitado que tem por índice exatamente a soma dos índices dos operadores presentes na composiçāo. Encerramos o capítulo dedicado aos operadores de Fredholm enunciando este resultado.

Teorema 2.9. Sejam $X, Y$ e $Z$ espaços de Banach. Se $S \in \mathcal{L}(X, Y)$ e $T \in \mathcal{L}(Y, Z)$ são operadores semi-Fredholm e seus indices podem ser somados, entäo $T S \in \mathcal{L}(X, Z)$ é semiFredholm com ind $(T S)=$ ind $T+$ ind $S$. 
Demonstração. Faremos a demonstração assumindo inicialmente que cada um dos operadores $T$ e $S$ possui nulidade ou deficiência igual a zero. Há, portanto, quatro casos a distinguir:

caso 1: $\operatorname{codim} \mathcal{R}(T)=0, \operatorname{codim} \mathcal{R}(S)=0$. Como $T$ e $S$ são sobrejetoras, $T S$ é sobrejetora e, além disso, $\operatorname{dim} \mathcal{N}(T S)=\operatorname{dim} \mathcal{N}(T)+\operatorname{dim} \mathcal{N}(S)$, quer este número seja finito ou não. Segue que $\operatorname{ind}(T S)=\operatorname{ind} T+$ ind $S$.

caso 2: $\operatorname{dim} \mathcal{N}(T)=0, \operatorname{dim} \mathcal{N}(S)=0$. Claramente $\operatorname{dim} \mathcal{N}(T S)=0$. Como $\mathcal{R}(T)$ e $\mathcal{R}(S)$ são fechadas, existem $c_{T}>0$ e $c_{S}>0$ que cumprem

$$
\|T x\| \geq c_{T}\|x\|, x \in X \quad \text { e } \quad\|S y\| \geq c_{S}\|y\|, y \in Y
$$

Em particular,

$$
\|T S x\| \geq c_{S} c_{T}\|x\|, x \in X
$$

com o que provamos que $\mathcal{R}(T S)$ é fechada.

Neste caso, verificar que ind $(T S)=\operatorname{ind} T+$ ind $S$ equivale a verificar que $\operatorname{codim} \mathcal{R}(T S)=$ $\operatorname{codim} \mathcal{R}(T)+\operatorname{codim} \mathcal{R}(S)$. Se $\operatorname{codim} \mathcal{R}(T)=\infty$ ou $\operatorname{codim} \mathcal{R}(S)=\infty$ é imediata a conclusão de que $\operatorname{codim} \mathcal{R}(T S)=\infty$.

Suponha então que $T$ e $S$ são operadores de Fredholm. Sob tal hipótese sabemos que $Y=$ $Y_{1} \oplus Y_{2}$ e $Z=Z_{1} \oplus Z_{2}$, onde $Y_{1}=\mathcal{R}(S), Z_{1}=\mathcal{R}(T)$ e $Y_{1}$ e $Z_{2}$ são subespaços de dimensão finita. De $\mathcal{N}(T)=\{0\}$ e $\operatorname{dim} Y_{2}<\infty$ obtemos que $Z=Z_{1} \oplus Z_{2}=T(R(S)) \oplus T\left(Y_{2}\right) \oplus Z_{2}$, com $\operatorname{dim} T\left(Y_{2}\right)=\operatorname{dim} Y_{2}$. Logo, $\operatorname{codim} \mathcal{R}(T S)=\operatorname{dim} T\left(Y_{2}\right)+\operatorname{dim} Z_{2}=\operatorname{codim} \mathcal{R}(S)+\operatorname{codim} \mathcal{R}(T)$. caso 3: $\operatorname{dim} \mathcal{N}(T)=0, \operatorname{codim} \mathcal{R}(S)=0$. Aqui, $\operatorname{dim} \mathcal{N}(T S)=\operatorname{dim} \mathcal{N}(S)=$ ind $S$ e $\operatorname{codim} \mathcal{R}(T S)=$ $\operatorname{codim} \mathcal{R}(T)=\operatorname{ind} T$. Se os índices de $T$ e $S$ podem ser somados, ou seja, se um dos operadores $T$ e $S$ possui índice finito, então $T S$ é semi-Fredholm, com ind $(T S)=\operatorname{ind} T+\operatorname{ind} S$.

caso 4: $\operatorname{codim} \mathcal{R}(T)=0, \operatorname{dim} \mathcal{N}(S)=0$. Sejam $Y_{1}=\mathcal{R}(S) \cap \mathcal{N}(T), Y_{2}$ um subespaço tal que $\mathcal{N}(T)=Y_{1} \oplus Y_{2}$ e $Y_{3}$ um subespaço satisfazendo $Y=\mathcal{R}(S) \oplus Y_{2} \oplus Y_{3}$. Desde que $T$ é sobrejetora,

$$
Z=T\left(\mathcal{R}(S) \oplus Y_{2} \oplus Y_{3}\right)=\mathcal{R}(T S) \oplus T\left(Y_{3}\right)
$$

o que torna evidente a desigualdade $\operatorname{codim} \mathcal{R}(S) \geq \operatorname{codim} \mathcal{R}(T S)$.

Para que os índices de $T$ e $S$ possam ser somados, pelo menos um deles deve ser finito.

Se ind $T=\infty$, necessariamente $\operatorname{codim} \mathcal{R}(S)<\infty$ e, pela discussão inicial, $\operatorname{codim} \mathcal{R}(T S)<$ $\infty$. Além disso, $\operatorname{dim} \mathcal{N}(T)=\infty$, do que concluímos que $T S$ é semi-Fredholm com ind $(T S)=\infty$.

Se, por outro lado, ind $T<\infty, \operatorname{dim} \mathcal{N}(T)=\operatorname{ind} T<\infty$. Nestas circunstâncias, $\operatorname{dim} Y_{2}<\infty$ e, conseqüentemente, $\operatorname{codim} \mathcal{R}(T S)=\operatorname{codim} \mathcal{R}(S)-\operatorname{dim} Y_{2}$. Ainda, pela injetividade de $S$ temos 
$\operatorname{dim} \mathcal{N}(T S)=\operatorname{dim} Y_{1}<\infty$. Portanto, $T S$ é semi-Fredholm e

$$
\begin{aligned}
\operatorname{ind}(T S) & =\operatorname{dim} Y_{1}-\left(\operatorname{codim} \mathcal{R}(S)-\operatorname{dim} Y_{2}\right)= \\
& =\left(\operatorname{dim} Y_{1}+\operatorname{dim} Y_{2}\right)-\operatorname{codim} \mathcal{R}(S) \\
& =\operatorname{dim} \mathcal{N}(T)-\operatorname{codim} \mathcal{R}(S) \\
& =\operatorname{ind} T+\operatorname{ind} S .
\end{aligned}
$$

Cuidemos agora do caso geral. Se $S \in \mathcal{L}(X, Y)$ é semi-Fredholm com índice menor ou igual a zero devemos ter $\mathcal{N}(S)$ de dimensão finita, digamos $m$, e $\operatorname{codim} \mathcal{R}(S) \geq m$. Escolha um subespaço $m$ dimensional $Y_{1} \subset Y \operatorname{com} Y_{1} \cap \mathcal{R}(S)=\{0\}$ e um isomorfismo $S_{1}$ entre $\mathcal{N}(S)$ e $Y_{1}$. Se $X_{1}=\mathcal{N}(S)$, escolha um subespaço fechado $X_{2}$ que verifique $X=X_{1} \oplus X_{2}$ e seja $G: X \longrightarrow Y$ o operador de posto finito (e portanto compacto) dado por $G\left(x_{1}+x_{2}\right)=S_{1} x_{1}$. O operador $S+G$ é injetor. De fato, $(S+G)\left(x_{1}+x_{2}\right)=0 \Longleftrightarrow S x_{2}+S_{1} x_{1}=0 \Longleftrightarrow S x_{2}=S_{1} x_{1}=0 \Longleftrightarrow x_{2}=x_{1}=0$.

Por outro lado, se o índice de $S$ é maior ou igual a zero então $\operatorname{codim} \mathcal{R}(S)=m$ para algum inteiro positivo $m$ e $\operatorname{dim} \mathcal{N}(S) \geq m$. Escolha agora subespaços $X_{1} \subset X$ e $Y_{1} \subset Y$, de dimensão finita $m$, tais que $X_{1} \subset \mathcal{N}(S)$ e $Y_{1} \cap \mathcal{R}(S)=\{0\}$. Note que $Y=Y_{1} \oplus \mathcal{R}(S)$ e existe subespaço fechado $X_{2} \subset X$ satisfazendo $X=X_{1} \oplus X_{2}$. Tome um isomorfismo $S_{1}$ entre os espaços $X_{1}$ e $Y_{1}$ e defina $L: X \longrightarrow Y$ por $L\left(x_{1}+x_{2}\right)=S_{1} x_{1}$. Por sua construção $L$ é compacto e $S+L$ é sobrejetor.

Dessa forma, se $T \in \mathcal{L}(Y, Z)$ e $S \in \mathcal{L}(X, Y)$ são operadores semi-Fredholm para os quais a soma ind $T+$ ind $S$ está definida, podemos escolher $L_{1} \in \mathcal{L}(Y, Z)$ e $L_{2} \in \mathcal{L}(X, Y)$, compactos, tais que cada um dos operadores $T+L_{1}$ e $S+L_{2}$ é injetor ou sobrejetor. Pelo que provamos anteriormente, $\left(T+L_{1}\right)\left(S+L_{2}\right)$ é semi-Fredholm com índice ind $\left(T+L_{1}\right)+\operatorname{ind}\left(S+L_{2}\right)$. Por fim, a observação de que $K=L_{1} S+T L_{2}+L_{1} L_{2}$ é um operador cuja imagem tem dimensão finita (e portanto compacto) nos conduz, se aplicamos o Teorema 2.8 , à conclusão de que $T S=$ $\left(T+L_{1}\right)\left(S+L_{2}\right)-K$ é semi-Fredholm e, mais ainda,

$$
\operatorname{ind}(T S)=\operatorname{ind}\left(T+L_{1}\right)+\operatorname{ind}\left(S+L_{2}\right)=\operatorname{ind} T+\operatorname{ind} S .
$$




\section{Capítulo 3}

\section{Perturbação de Contorno}

Este capítulo compõe-se de duas seções nas quais apresentamos técnicas e ferramentas empregadas para lidar com o problema da pertubação dos domínios de definição de um problema de contorno. Na primeira seção exporemos um tipo de cálculo diferencial relacionado às pertubações de fronteira. Na segunda, enunciaremos e provaremos uma generalização, obtida por D. Henry, do Teorema da Transversalidade estabelecido por Abraham e Thom.

\subsection{Cálculo Diferencial das Perturbações de Fronteira}

Nesta seçāo abordaremos um tipo de cálculo em que pretendemos que a variável independente seja o domínio de definição de um problema de contorno.

Há duas maneiras usuais de descrever a deformação de uma região:

(i)descrição lagrangeana: dada uma região inicial $\Omega \subset \mathbb{R}^{n}$, temos uma aplicação $(t, p) \longmapsto x(t, p)$ que fornece a posição da partícula $p \in \Omega$ no instante $t$.

(ii)descrição euleriana: escrevemos a velocidade e outras variáveis como função do tempo e da posição em um sistema de coordenadas fixado. Nesta forma temos, por exemplo, uma função velocidade $V(t, x)$ que indica a velocidade que uma partícula teria no ponto $x$, no instante $t$. Se $x(t, p)$ é a posição que a partícula $p \in \Omega$ ocupa no instante $t$, cntão

$$
\frac{\partial x}{\partial t}(t, p)=V(t, x(t, p))
$$

Vamos considerar um operador diferencial não linear $u \longmapsto v$,

$$
v(y)=f\left(y, u(y), \frac{\partial u}{\partial y_{1}}(y), \ldots, \frac{\partial u}{\partial y_{n}}(y), \frac{\partial^{2} u}{\partial y_{1}^{2}}(y), \frac{\partial^{2} u}{\partial y_{2} \partial y_{1}}(y), \ldots\right), \quad y \in \mathbb{R}^{n},
$$

em que $f$ é uma função fixada.

Para tornar a notação mais compacta, definiremos um operador diferencial linear $L$ por

$$
L u(y)=\left(u(y), \frac{\partial u}{\partial y_{1}}(y), \ldots, \frac{\partial u}{\partial y_{n}}(y), \frac{\partial^{2} u}{\partial y_{1}^{2}}(y), \frac{\partial^{2} u}{\partial y_{2} \partial y_{1}}(y), \ldots\right)
$$


com tantos termos quantos forem necessários, de modo que o operador diferencial não linear inicial assume a forma

$$
u(\cdot) \longmapsto f(\cdot, L u(\cdot))
$$

Mais precisamente, suponha $f: U \subset \mathbb{R}^{n} \times \mathbb{R}^{p} \longrightarrow \mathbb{R}$ uma aplicação definida em um aberto $U, \Omega$ um aberto de $\mathbb{R}^{n}$ e admita ainda que $L u(y) \in \mathbb{R}^{p}$ onde estiver definido. Para funções $u: \Omega \longrightarrow \mathbb{R}$ suficientemente suaves, tais que $(x, L u(x)) \in U$ para todo $x \in \bar{\Omega}$, definimos $F_{\Omega}$ por

$$
F_{\Omega}(u)(x)=f(x, L u(x)), \quad x \in \Omega
$$

Se, para sermos mais específicos, $f$ é uma função contínua, $\Omega$ é limitado e o operador linear $L$ envolve derivadas até ordem $m$, o domínio de $F_{\Omega}$ será um subconjunto aberto de $C^{m}(\Omega)$ (possivelmente vazio) e os valores de $F_{\Omega}$ estarão em $C^{0}(\Omega)$. Naturalmente, outros espaços de funções podem ser utilizados, como os espaços de Sobolev $W^{m, p}(\Omega)$, por exemplo.

Dizemos que uma aplicação $h: \Omega \longrightarrow \mathbb{R}^{n}$ é um mergulho de classe $C^{m}$ se $h$ é um difeomorfismo de classe $C^{m}$ de $\Omega$ sobre $h(\Omega)$. Dado um mergulho $h: \Omega \longrightarrow \mathbb{R}^{n}$, definimos o pull-back $h^{*}$ por

$$
h^{*} u(x)=u(h(x)), \quad x \in \Omega,
$$

que aplicamos a funções $u$ definidas em $h(\Omega)$. Claramente, $h^{*}$ é um isomorfismo de $C^{m}(h(\Omega))$ sobre $C^{m}(\Omega)$, cuja inversa é $\left(h^{-1}\right)^{*}$. Usamos a mesma notação para o pull-back em outros espaços de funções. Assim, escrevemos $h^{*}: W^{m, p}(h(\Omega)) \rightarrow W^{m, p}(\Omega)$ para espaços de Sobolev, $h^{*}: C^{k, \beta}(h(\Omega)) \longrightarrow C^{k, \beta}(\Omega), 0 \leq k+\beta \leq m$, para os espaços de Hölder, etc.

No caso em que $\Omega$ é limitado e o domínio $\mathcal{D}\left(F_{\Omega}\right)$ de $F_{\Omega}$ é não vazio, para $h \in \operatorname{Diff}^{m}(\Omega)$ em uma vizinhança suficientemente pequena de $i_{\Omega}$, o domínio de $F_{h(\Omega)}$ é também não-vazio. De fato, se $v \in \mathcal{D}\left(F_{\Omega}\right)$ então $u=h^{*-1} v \in \mathcal{D}\left(F_{h(\Omega)}\right)$, para $h$ na vizinhança em questão.

Ao passarmos da região $\Omega$ para a região $h(\Omega)$ por meio do mergulho $h$, podemos visualizar o operador diferencial não-linear formal $v(\cdot) \longmapsto f(\cdot, L v(\cdot))$ em $h(\Omega)$ de duas maneiras: o operador não linear

$$
F_{h(\Omega)}: \mathcal{D}\left(F_{h(\Omega)}\right) \subset C^{m}(h(\Omega)) \longrightarrow C^{0}(h(\Omega))
$$

é sua forma euleriana, enquanto a forma lagrangeana corresponde ao operador

$$
h^{*} F_{h(\Omega)} h^{*-1}: h^{*}\left(\mathcal{D}\left(F_{h(\Omega)}\right)\right) \subset C^{m}(\Omega) \longrightarrow C^{0}(\Omega),
$$

sendo empregada a mesma terminologia quando outros espaços de funçōes substituem $C^{m}(h(\Omega))$ e $C^{m}(\Omega)$.

A forma lagrangeana apresenta a conveniência de atuar em espaços que independem de $h$, além de servir a nosso propósito de calcular derivadas com respeito aos domínios: um modo natural de fazê-lo é tomar derivadas com respeito à variável $h$. 
Atendo-nos à forma lagrangeana, consideramos a aplicação

$$
F:(h, u) \longmapsto h^{*} F_{h(\Omega)} h^{*-1}(u)
$$

definida em

$$
\mathcal{D}(F)=\bigcup_{h \in \operatorname{Diff}^{m}(\Omega)}\left\{(h, u) ; h^{*-1} u \in \mathcal{D}\left(F_{h(\Omega)}\right)\right\},
$$

que é um aberto de $\operatorname{Diff}^{m}(\Omega) \times C^{m}(\Omega)$. Precisamos estudar sua diferenciabilidade e obter ferramentas para o cálculo de derivadas com respeito a $h$.

Observando que

$$
\begin{aligned}
h^{*} F_{h(\Omega)} h^{*-1}(u)(x) & =F_{h(\Omega)}\left(h^{*-1} u\right)(h(x)) \\
& =f\left(h(x), L\left(h^{*-1} u\right)(h(x))\right) \\
& =f\left(h(x), h^{*} L h^{*-1} u(x)\right), x \in \Omega,
\end{aligned}
$$

concluímos que é suficiente examinarmos a diferenciabilidade da aplicação

$$
(h, u) \longmapsto h^{*} L h^{*-1} u: \operatorname{Diff}^{m}(\Omega) \times C^{m}(\Omega) \longrightarrow C^{0}(\Omega) .
$$

Por se tratar de uma aplicação linear em $u$, nossa preocupação recai somente sobre as derivadas em $h$.

Levando em consideração que um operador diferencial linear formal $L$ tem o aspecto

$$
u(\cdot) \longmapsto\left(\left(\frac{\partial}{\partial y}\right)^{\alpha_{1}} u(\cdot), \ldots,\left(\frac{\partial}{\partial y}\right)^{\alpha_{k}} u(\cdot)\right)
$$

onde $\alpha_{i}=\left(\alpha_{i, 1}, \ldots, \alpha_{i, n}\right) \in \mathbb{N}^{n}$, com $\left|\alpha_{i}\right|=\sum_{j=1}^{n} \alpha_{i, j} \leq m$, para $i=1, \ldots, k$ e

$$
\left(\frac{\partial}{\partial y}\right)^{\alpha_{i}} u=\prod_{j=1}^{n}\left(\frac{\partial}{\partial y_{j}}\right)^{\alpha_{i, j}} u
$$

podemos supor que $L$ é simplesmente da forma

$$
u(\cdot) \longmapsto\left(\frac{\partial}{\partial y}\right)^{\alpha} u(\cdot)
$$

com $\alpha=\left(\alpha_{1}, \ldots, \alpha_{n}\right) \in N^{n}$ satisfazendo $|\alpha| \leq m$.

Neste caso, pela regra da cadeia, temos

$$
\begin{aligned}
h^{*}\left(\frac{\partial}{\partial y_{i}}\right) h^{*-1} u(x) & =\frac{\partial\left(u \circ h^{-1}\right)}{\partial y_{1}}(h(x)) \\
& =\sum_{j=1}^{n} \frac{\partial u}{\partial x_{j}}(x) \frac{\partial h_{j}^{-1}}{\partial y_{i}}(h(x)) \\
& =\sum_{j=1}^{n} \frac{\partial u}{\partial x_{j}}(x)\left[h^{\prime}(x)\right]_{j i}^{-1},
\end{aligned}
$$


onde $\left[h^{\prime}(x)\right]_{j i}^{-1}$ é o $j i$-ésimo elemento da inversa da matriz (nas bases canônicas) da derivada de $h$ no ponto $x$. Logo,

$$
\begin{aligned}
h^{*}\left(\frac{\partial}{\partial y}\right)^{\alpha} h^{*-1} u(x) & =\prod_{i=1}^{n}\left(h^{*}\left(\frac{\partial}{\partial y_{i}}\right) h^{*-1}\right)^{\alpha_{i}} u(x) \\
& =\prod_{i=1}^{n}\left(\sum_{j=1}^{n}\left[h^{\prime}(x)\right]_{j i}^{-1} \frac{\partial}{\partial x_{j}}\right)^{\alpha_{i}} u(x),
\end{aligned}
$$

do que segue a conclusão de que, na verdade, $(h, u) \longmapsto h^{*} L h^{*-1} u$ é analítica no aberto $\operatorname{Diff}^{m}(\Omega)$ em $h$. Conseqüentemente, se $f$ é de classe $C^{k}$ então $F: \mathcal{D}(F) \subset \operatorname{Diff}^{m}(\Omega) \times C^{m}(\Omega) \longrightarrow C^{0}(\Omega)$ também será de classe $C^{k}$. Os resultados de suavidade são essencialmente os mesmos quando são empregados outros espaços de funçōes.

Nossa intenção agora será a de calcular a derivada de Gâteaux de $h \longmapsto F(h, u)=h^{*} F_{h(\Omega)} h^{*-1}(u)$, ou seja, a $t$-derivada ao longo de uma curva de mergulhos $t \longmapsto h(t, \cdot)$ de classe $C^{1}$.

Suponha que desejamos calcular

$$
\frac{\partial}{\partial t} F_{\Omega(t)}(v)(y)=\frac{\partial}{\partial t} f(y, L v(y))
$$

com $y$ fixado em $\Omega(t)=h(t, \Omega)$. Como $y=h(t, x)$ para algum $x \in \Omega=\Omega(0)$, para mantê-lo fixo $x$ precisa se mover. Assim, devemos ter $y=h(t, x(t))$ com

$$
0=\frac{\partial h}{\partial t}+\frac{\partial h}{\partial x} x^{\prime}(t)
$$

de modo que se $U(x, t)=\left(\frac{\partial h}{\partial x}\right)^{-1} \frac{\partial h}{\partial t}, x(t)$ deve ser solução da equação diferencial $d x / d t=$ $-U(x, t)$. Com isso, a derivada parcial em $t$ na forma euleriana com $y=h(t, x)$ fixado corresponde à derivada anti-convectiva $D_{t}$ da forma Lagrangeana na região de referência $\Omega$ :

$$
D_{t}=\frac{\partial}{\partial t}-U(x, t) \cdot \frac{\partial}{\partial x}, \quad U(x, t)=\left(\frac{\partial h}{\partial x}\right)^{-1} \frac{\partial h}{\partial t} .
$$

Mais precisamente, temos o seguinte.

Lema 3.1. Suponha $\psi: \mathbb{R}^{n} \times \mathbb{R} \longrightarrow \mathbb{R}^{n}$ de classe $C^{1}, h: \mathbb{R}^{n} \times \mathbb{R} \longrightarrow \mathbb{R}$ de classe $C^{1}$ e que $\operatorname{det} h_{x}(x, t) \neq 0$ na região de interesse. Então

$$
D_{t}\left(h^{*}(t) \psi(\cdot, t)\right)=h^{*}(t) \frac{\partial \psi}{\partial t}(\cdot, t)
$$

onde $h^{*}(t)$ é o pull-back relativo a $h(\cdot, t)$.

Demonstraçäo. Seja $\Phi(\cdot, t)=h^{*}(t) \psi(\cdot, t)$, ou seja, $\Phi(x, t)=\psi(h(x, t), t)$. Pela regra da cadeia,

$$
\begin{aligned}
\frac{\partial \Phi}{\partial t}(x, t) & =\frac{\partial \psi}{\partial x}(h(x, t), t) \frac{\partial h}{\partial t}(x, t)+\frac{\partial \psi}{\partial t}(h(x, t), t) \\
& =\sum_{j=1}^{n} \frac{\partial \psi}{\partial x_{j}}(h(x, t), t) \frac{\partial h_{j}}{\partial t}(x, t)+\frac{\partial \psi}{\partial t}(h(x, t), t)
\end{aligned}
$$


e

$$
U(x, t) \cdot \frac{\partial \Phi}{\partial x}(x, t)=\sum_{k=1}^{n} U_{k}(x, t) \frac{\partial \Phi}{\partial x_{k}}(x, t)=\sum_{k=1}^{n} U_{k}(x, t)\left(\sum_{j=1}^{n} \frac{\partial \psi}{\partial y_{j}}(h(x, t), t) \frac{\partial h_{j}}{\partial x_{k}}(x, t)\right)
$$

Assim,

$$
\begin{aligned}
& D_{t}\left(h^{*}(t) \psi(\cdot, t)\right)(x)=\frac{\partial \Phi}{\partial t}(x, t)-U(x, t) \cdot \frac{\partial \Phi}{\partial x}(x, t) \\
& =\sum_{j=1}^{n} \frac{\partial \psi}{\partial x_{j}}(h(x, t), t) \frac{\partial h_{j}}{\partial t}(x, t)+\frac{\partial \psi}{\partial t}(h(x, t), t)-\sum_{k+1}^{n} U_{k}(x, t)\left(\sum_{j=1}^{n} \frac{\partial \psi}{\partial y_{j}}(h(x, t), t) \frac{\partial h_{j}}{\partial x_{k}}(x, t)\right) \\
& =\sum_{j=1}^{n} \frac{\partial \psi}{\partial y_{j}}(h(x, t), t)\left(\frac{\partial h_{j}}{\partial t}(x, t)-\sum_{k=1}^{n} U_{k}(x, t) \frac{\partial h_{j}}{\partial x_{k}}(x, t)\right)+\frac{\partial \psi}{\partial t}(h(x, t), t) .
\end{aligned}
$$

Agora,

$$
U_{k}(x, t)=\sum_{i=1}^{n}\left(h_{x}(x, t)\right)_{k i}^{-1} \frac{\partial h_{i}}{\partial t}(x, t)
$$

resultando que

$$
\begin{aligned}
\sum_{k=1}^{n} U_{k}(x, t) \frac{\partial h_{j}}{\partial x_{k}}(x, t) & =\sum_{k=1}^{n}\left(\sum_{i=1}^{n}\left(h_{x}(x, t)\right)_{k i}^{-1} \frac{\partial h_{i}}{\partial t}(x, t)\right) \frac{\partial h_{j}}{\partial x_{k}}(x, t) \\
& =\sum_{i=1}^{n} \frac{\partial h_{i}}{\partial t}(x, t)\left(\sum_{k=1}^{n}\left(h_{x}(x, t)\right)_{k i}^{-1} \frac{\partial h_{j}}{\partial x_{k}}(x, t)\right) \\
& =\sum_{i=1}^{n} \frac{\partial h_{i}}{\partial t}(x, t) \delta_{i j}=\frac{\partial h_{j}}{\partial t}(x, t) .
\end{aligned}
$$

Portanto,

$$
\begin{aligned}
D_{t}\left(h^{*}(t) \psi(\cdot, t)\right)(x) & =\frac{\partial \psi}{\partial t}(h(\cdot, t), t)(x) \\
& =\left(h^{*}(t) \frac{\partial \psi}{\partial t}(\cdot, t)\right)(x),
\end{aligned}
$$

o que significa precisamente que

$$
D_{t}\left(h^{*}(t) \psi(\cdot, t)\right)=h^{*}(t) \frac{\partial \psi}{\partial t}(\cdot, t)
$$

Teorema 3.2. Suponha $f(t, y, \lambda)$ de classe $C^{1}$ em um aberto de $\mathbb{R} \times \mathbb{R}^{n} \times \mathbb{R}^{p}, L$ um operador diferencial com coeficientes constantes de ordem $\leq m$, com $L v(y) \in \mathbb{R}^{p}$ onde estiver definido, $e$ para conjuntos $Q \subset \mathbb{R}^{n}$ e funções $v$ de classe $C^{m}$ em $Q$, seja $F_{Q}(t) v$ a função

$$
\left(F_{Q}(t) v\right)(y)=f(t, y, L v(y)), y \in Q
$$

para as funçōes $v$ em que $F_{Q}(t)$ estiver definida. 
Suponha $t \longmapsto h(t, \cdot)$ uma curva de mergulhos de um aberto $\Omega \subset \mathbb{R}^{n}, \Omega(t)=h(t, \Omega)$ e que para $|j| \leq m,|k| \leq m+1,(t, x) \longmapsto \partial_{t} \partial_{x}^{j} h(t, x), \partial_{x}^{k} h(t, x), \partial_{t} \partial_{x}^{j} u(t, x), \partial_{x}^{k} u(t, x)$ são contínuas em $\mathbb{R} \times \Omega$ próximo de $t=0, e$

$$
\left(h^{*}(t)\right)^{-1} u(t, \cdot)
$$

está no domínio de $F_{\Omega(t)}(t)$. Então, em pontos de $\Omega$,

$\left.D_{t}\left(h^{(} t\right) * F_{\Omega(t)}\left(h^{*}(t)\right)^{-1}\right)(u)=\left(h^{*}(t) \dot{F}_{\Omega(t)}\left(h^{*}(t)\right)^{-1}\right)(u)+\left(h^{*}(t) F_{\Omega(t)}^{\prime}(t)\left(h^{*}(t)\right)^{-1}\right)(u)\left(\left(h^{*}(t)\right)^{-1} D_{t} u\right)$

onde $D_{t}$ é a derivada anti-convectiva,

$$
\left(\dot{F}_{Q}(t) v\right)(y)=\frac{\partial f}{\partial t}(t, y, L v(y)), y \in Q
$$

$e$

$$
\left(F_{Q}^{\prime}(t) v \cdot w\right)(y)=\frac{\partial f}{\partial \lambda}(t, y, L v(y)) L w(y), y \in Q
$$

é a linearização de $v \mapsto F_{Q}(t) v$.

Demonstração. Seja $v(t, \cdot)=\left(h^{*}(t)\right)^{-1} u(t, \cdot)$. Pelo Lema 3.1, temos

$$
\begin{aligned}
D_{t}\left(h^{*}(t) L\left(h^{*}(t)\right)^{-1}\right)(u(t, \cdot)) & =D_{t}\left(h^{*}(t) L v(t, \cdot)\right) \\
& =h^{*}(t) L \frac{\partial v}{\partial t}(t, \cdot)=h^{*}(t) L\left(h^{*}(t)\right)^{-1} D_{t} u(t, \cdot),
\end{aligned}
$$

já que

$$
D_{t} u(t, \cdot)=D_{t}\left(h^{*} v(t, \cdot)\right)=h^{*}(t) \frac{\partial v}{\partial t}(t, \cdot)
$$

Logo,

$$
\begin{aligned}
D_{t}\left(h^{*}(t) F_{\Omega(t)}(t)\left(h^{*}(t)\right)^{-1} u(t, \cdot)\right)= & D_{t}\left(h^{*}(t) f(t, \cdot, L v(t, \cdot))\right) \\
= & h^{*}(t) \frac{\partial}{\partial t} f(t, \cdot L v(t, \cdot)) \\
= & h^{*}(t)\left[\frac{\partial f}{\partial t}(t, \cdot L v(t, \cdot))+\frac{\partial f}{\partial \lambda}(t, \cdot, L v(t, \cdot)) L \frac{\partial v}{\partial t}(t, \cdot)\right] \\
= & h^{*}(t) \frac{\partial f}{\partial t}(t, \cdot L v(t, \cdot)) \\
& +\frac{\partial f}{\partial \lambda}(t, \cdot L v(t, \cdot)) L\left(\left(h^{*}(t)\right)^{-1} D_{t} u(t, \cdot)\right) \\
= & h^{*}(t) \frac{\partial f}{\partial t}\left(t, \cdot L\left(\left(h^{*}(t)\right)^{-1} u(t, \cdot)\right)\right) \\
& +\frac{\partial f}{\partial \lambda}\left(t, \cdot, L\left(\left(h^{*}(t)\right)^{-1} u(t, \cdot)\right)\right) L\left(\left(h^{*}(t)\right)^{-1} D_{t} u(t, \cdot)\right) \\
= & h^{*}(t) \dot{F}_{\Omega(t)}(t)\left(h^{*}(t)\right)^{-1} u(t, \cdot) \\
& \left.+h^{*}(t)\left(F_{\Omega(t)}^{\prime}(t)\left(h^{*}(t)\right)^{-1} u(t, \cdot)\right)\right)\left(\left(h^{*}(t)\right)^{-1} D_{t} u(t, \cdot)\right) .
\end{aligned}
$$


Exemplo: Suponha que estejamos lidando com o operador linear

$$
A=\sum_{|\alpha| \leq m} a_{\alpha}(y)\left(\frac{\partial}{\partial y}\right)^{\alpha},
$$

que não depende explicitamente de $t$ e seja $h(t, x)=x+t V(x)$, para $t$ em uma vizinhança de 0 e $x \in \Omega$. Então, pelo Teorema 3.2, temos

$$
\begin{aligned}
\left.\frac{\partial}{\partial t}\left(h^{*} A h^{*-1} u\right)\right|_{t=0} & =\left.D_{t}\left(h^{*} A h^{*-1} u\right)\right|_{t=0}+\left.h_{x}^{-1} h_{t} \nabla\left(h^{*} A h^{*-1} u\right)\right|_{t=0} \\
& =A\left(\frac{\partial u}{\partial t}-V \cdot \nabla u\right)+V \cdot \nabla(A u) \\
& =A \frac{\partial u}{\partial t}+[V \cdot \nabla, A] u .
\end{aligned}
$$

O comutador de $V \cdot \nabla$ e $A$, que indicamos acima por $[V \cdot \nabla, A]$ é ainda um operador de ordem $m$, embora $A V \cdot \nabla$ e $V \cdot \nabla A$ sejam operadores de ordem $m+1$, podendo ser calculado em funções de classe $C^{m}$.

Até agora vimos adotando $\Omega$ como "origem" ou região de referência, mas podemos transferila facilmente para qualquer região $\Omega_{1}$ difeomorfa a $\Omega$. Seja $H_{1}: \Omega \longrightarrow \Omega_{1}$ um difeomorfismo. Para cada mergulho $h: \Omega_{1} \rightarrow \mathbb{R}^{n}$, defina o mergulho $h_{1}=h \circ H_{1}$. Da mesma forma, defina $x_{1}=H_{1}^{-1} x$ e $u_{1}=u \circ H_{1}$. Entāo

$$
h^{*} F_{h\left(\Omega_{1}\right)} h^{*-1} u(x)=h_{1}^{*} F_{h_{1}(\Omega)} h_{1}^{*-1} u_{1}\left(x_{1}\right) .
$$

Esta mudança de origem nos permitirá calcular derivadas com respeito à variável $h$ em $h=i_{\Omega}$, onde os cálculos são mais simples.

\subsection{Teorema da Transversalidade}

Reservamos esta seção para a apresentação do enunciado e da demonstração de uma importante generalização do Teorema da Transversalidade de Abraham e Thom [1], que D. Henry prova em [12] visando aplicações em dimensão infinita, com ênfase para o caso de índice negativo.

Esta generalização, bem como o cálculo diferencial desenvolvido na seção precedente, são essenciais na obtenção do resultado sobre hiperbolicidade genérica exposto na última seção do Capítulo 4.

Antes que enunciemos o resultado algumas definições são necessárias.

Um subconjunto $S$ de um espaço topológico $X$ é magro (em $X$ ) quando é uma reuniāo enumerável $S=\cup_{n=1}^{\infty} S_{n}$ tal que, para cada $n$, o fecho de $S_{n}$ tem interior vazio.

Para que $S$ seja magro em $X$ é condição necessária e suficiente que tenhamos $S \subset \cup_{n=1}^{\infty} F_{n}$, onde $F_{1}, F_{2}, \ldots, F_{n}, \ldots$ sāo fechados com interior vazio. 
Uma propriedade que se infere imediatamente da definição anterior é que a reunião de uma família enumerável de subconjuntos magros em $X$ resulta ainda em um subconjunto magro em $X$.

Dizemos que um subconjunto $S$ é residual se o seu complementar é magro, ou seja, se $S$ contém uma intersecção enumerável de abertos densos.

Se $X$ e $Y$ são espaços de Banach, $U \subset X$ é um aberto e $f: X \rightarrow Y$ é uma aplicação de classe $C^{k}(k \geq 1)$, diremos que um ponto $x \in U$ é um ponto regular de $f$ se a derivada $f^{\prime}(x)$ é sobrejetora e seu núcleo admite um complementar fechado. Um valor crítico de $f$ é a imagem, por $f$, de algum ponto crítico de $f$. Todos os demais pontos de $Y$ são chamados valores regulares de $f$, incluindo aqueles no complementar da imagem de $f$.

Em nossas aplicações, o núcleo de $f^{\prime}(x)$ sempre terá dimensão finita, de modo que $x$ será um ponto crítico de $f$ se, e somente se, $f^{\prime}(x)$ não for sobrejetora.

Teorema 3.3. (Teorema da Transversalidade) Sejam $k$ um inteiro positivo, $X, Y$ e $Z$ variedades de Banach de classe $C^{k}, A \subset X \times Y$ aberto, $f: A \longrightarrow Z$ aplicação de classe $C^{k} e$ $\xi \in Z$. Suponha que para todo $(x, y) \in f^{-1}(\xi)$ tenhamos:

(1) $\frac{\partial J}{\partial x}(x, y): T_{x} X \longrightarrow T_{\xi} Z$ é semi-Fredholm com índice $<k$.

(2) Uma das seguintes alternativas é válida:

(a) $D f(x, y)=\left(\frac{\partial f}{\partial x}(x, y), \frac{\partial f}{\partial y}(x, y)\right): T_{x} X \times T_{y} Y \longrightarrow T_{\xi} Z$ é sobrejetora, ou

(b) $\operatorname{dim}\left\{\mathcal{R}(D f(x, y)) / \mathcal{R}\left(\frac{\partial f}{\partial x}(x, y)\right)\right\} \geq 1+\operatorname{dim} \mathcal{N}\left(\frac{\partial f}{\partial x}(x, y)\right)$.

Suponha ainda que

(3) $(x, y) \longmapsto y: f^{-1}(\xi) \longrightarrow Y$ é uma aplicação $\sigma-$ própria, ou seja, $f^{-1}(\xi)=\cup_{j=1}^{\infty} M_{j}$ é uma reunião enumerável de conjuntos $M_{j}$ tais que $(x, y) \longmapsto Y: M_{j} \longrightarrow y$ é uma aplicação própria para cada $j$.

$$
\begin{aligned}
& \operatorname{Sejam} A_{y}=\{x \in X ;(x, y) \in A\} \text { e } \\
& \qquad Y_{\text {crit }}=\left\{y \in Y ; \xi \text { é um valor crítico de } f(\cdot, y): A_{y} \longrightarrow Z\right\} .
\end{aligned}
$$

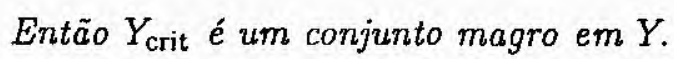

Antes de apresentarmos a demonstração do teorema acima julgamos oportuno fazer duas observações sobre o seu enunciado.

Observação 1: Se $f^{-1}(\xi)$ é um espaço de Lindelöf (ou seja, se toda cobertura aberta de $f^{-1}(\xi)$ admite subcobertura enumerável) e em todo ponto $(x, y) \in f^{-1}(\xi)$ a hipótese (1) está satisfeita, entāo a hipótese (3) estará automaticamente satisfeita. Provaremos esta afirmação no Lema 3.5 que apresentamos a seguir. 
Uma situação particular em que $f^{-1}(\xi)$ é um espaço de Lindelöf é aquela em que $X$ e $Y$ são espaços métricos separáveis.

Observação 2: A hipótese (2)(b) é mais geral para o caso de índice negativo. Corn efeito, se a hipótese (2)(b) se verifica em um ponto $(x, y) \in f^{-1}(\xi)$, como

$$
\operatorname{codim} \mathcal{R}\left(\frac{\partial f}{\partial x}(x, y)\right) \geq \operatorname{dim}\left\{\mathcal{R}(D f(x, y)) / \mathcal{R}\left(\frac{\partial f}{\partial x}(x, y)\right)\right\}
$$

temos que

$$
\text { ind }\left(\frac{\partial f}{\partial x}(x, y)\right) \leq \operatorname{dim} \mathcal{N}\left(\frac{\partial f}{\partial x}(x, y)\right)-\operatorname{dim}\left\{\mathcal{R}(D f(x, y)) / \mathcal{R}\left(\frac{\partial f}{\partial x}(x, y)\right)\right\} \leq-1
$$

Sendo assim, a hipótese (2)(b) só pode ser verdadeira em um ponto $(x, y) \in f^{-1}(\xi)$ se o índice de $\frac{\partial f}{\partial x}(x, y)$ é negativo.

Por outro lado, se o índice de $\frac{\partial f}{\partial x}(x, y)$ é negativo e a hipótese (2)(a) vale, então

$$
\operatorname{codim} \mathcal{R}\left(\frac{\partial f}{\partial x}(x, y)\right)=\operatorname{dim}\left\{\mathcal{R}(D f(x, y)) / \mathcal{R}\left(\frac{\partial f}{\partial x}(x, y)\right)\right\}
$$

e

resultando que

$$
\operatorname{dim} \mathcal{N}\left(\frac{\partial f}{\partial x}(x, y)\right)-\operatorname{dim}\left\{\mathcal{R}(D f(x, y)) / \mathcal{R}\left(\frac{\partial f}{\partial x}(x, y)\right)\right\} \leq-1
$$

$$
\operatorname{dim}\left\{\mathcal{R}(D f(x, y)) / \mathcal{R}\left(\frac{\partial f}{\partial x}(x, y)\right)\right\} \geq 1+\operatorname{dim} \mathcal{N}\left(\frac{\partial f}{\partial x}(x, y)\right)
$$

Fracionaremos a demonstração do Teorema 3.3 em vários resultados auxiliares.

Lema 3.4. Sejam $X, Y, Z$ variedades de Banach de classe $C^{1}$ e $f$ uma aplicação de um aberto $A \subset X \times Y$ em $Z$. Suponha $\left(x_{0}, y_{0}\right)$ um ponto de $A$ tal que $f$ é de classe $C^{1}$ em uma vizinhança $W_{0}$ de $\left(x_{0}, y_{0}\right), f\left(x_{0}, y_{0}\right)=\xi e \frac{\partial f}{\partial x}\left(x_{0}, y_{0}\right)$ é um operador semi-Fredholm à esquerda. Então existe vizinhança $W$ de $\left(x_{0}, y_{0}\right)$, com $\bar{W} \subset W_{0}$, tal que $(x, y) \longmapsto y: f^{-1}(\xi) \cap \bar{W} \longrightarrow Y$ é aplicação própria.

Demonstração. Sendo o resultado local, podemos supor que $X, Y$ e $Z$ são espaços de Banach. A hipótese de que $L=\frac{\partial f}{\partial x}\left(x_{0}, y_{0}\right)$ é semi-Fredholm à esquerda fornece que $X_{1}=\mathcal{N}(L)$ tem dimensão finita, de modo que existe subespaço fechado $X_{2} \subset X$ tal que $X=X_{1} \oplus X_{2}$ e a restrição de $L$ a $X_{2}$ é um isomorfismo de $X_{2}$ em $\mathcal{R}(L)$. Observe que a inversa de tal restrição é contínua já que $\mathcal{R}(L)$ è subespaço fechado. Assim, existe $c>0$ satisfazendo $\left\|L x_{2}\right\| \geq c\left\|x_{2}\right\|$ para todo $x_{2} \in X_{2}$.

Como $f$ é de classe $C^{1}$, se $B=1+\left\|\frac{\partial f}{\partial x}\left(x_{0}, y_{0}\right)\right\|$, existe uma vizinhança $W$ de $\left(x_{0}, y_{0}\right)$ satisfazendo $\bar{W} \subset W_{0}$ e

$$
\left\|f(x, y)-f\left(x^{\prime}, y^{\prime}\right)-L\left(x-x^{\prime}\right)\right\| \leq \frac{c}{2}\left\|x-x^{\prime}\right\|+B\left\|y-y^{\prime}\right\|,
$$


quaisquer que sejam $(x, y)$ e $\left(x^{\prime}, y^{\prime}\right)$ em $W$.

Considere $\left\{\left(x_{n}, y_{n}\right)\right\}$ sequência em $f^{-1}(\xi) \cap \bar{W}$ para a qual $y_{n} \rightarrow y$ em $Y$. Então

$$
\left\|L\left(x_{n}-x_{m}\right)\right\|=\left\|f\left(x_{n}, y_{n}\right)-f\left(x_{m}, y_{m}\right)-L\left(x_{n}-x_{m}\right)\right\| \leq \frac{c}{2}\left\|x_{n}-x_{m}\right\|+B\left\|y_{n}-y_{m}\right\| .
$$

Escrevendo $x_{n}=x_{n}^{1}+x_{n}^{2}$, com $x_{n}^{1} \in X_{1}$ e $x_{n}^{2} \in X_{2}$, notamos que $\left\{x_{n}^{1}\right\}$ é uma sequência limitada em um espaço de dimensão finita (a limitação é consequência do fato de que, na verdade, $\left\{\left(x_{n}, y_{n}\right)\right\}$ é limitada). Então existe subsequência $\left\{x_{n_{k}}^{1}\right\}$ convergente. Mas

$$
\begin{aligned}
c\left\|x_{n_{k}}^{2}-x_{n_{l}}^{2}\right\| & \leq\left\|L\left(x_{n_{k}}^{2}-x_{n_{l}}^{2}\right)\right\|=\left\|L\left(x_{n_{k}}-x_{n_{l}}\right)\right\| \\
& \leq \frac{c}{2}\left\|x_{n_{k}}-x_{n_{l}}\right\|+B\left\|y_{n_{k}}-y_{n_{l}}\right\|
\end{aligned}
$$

resultando que

$$
\left\|x_{n_{k}}^{2}-x_{n_{l}}^{2}\right\| \leq\left\|x_{n_{k}}^{1}-x_{n_{l}}^{1}\right\|+\frac{2 B}{c}\left\|y_{n_{k}}-y_{n_{l}}\right\|,
$$

o que mostra que $\left\{x_{n_{k}}^{2}\right\}$ é sequência de Cauchy no espaço de Banach $X_{2}$. Segue que $\left\{x_{n_{k}}\right\}$ e $\left\{\left(x_{n_{k}}, y_{n_{k}}\right)\right\}$ são sequências convergentes. Se $(x, y)$ é o limite de $\left\{\left(x_{n_{k}}, y_{n_{k}}\right)\right\}$, a continuidade de $f$ e o fato de $\bar{W}$ ser fechado asseguram que $(x, y) \in f^{-1}(\xi) \cap \bar{W}$, o que completa a demonstração.

Lema 3.5. Sejam $k$ um inteiro positivo, $X, Y, Z$ variedades de Banach de classe $C^{k}, A \subset X \times Y$ um aberto, $f: A \longrightarrow Z$ uma aplicação de classe $C^{k}$ e $\xi$ um ponto de $Z$. Suponha que

(1) Para todo $(x, y) \in f^{-1}(\xi)$ o operador $\frac{\partial f}{\partial x}(x, y): T_{x} X \rightarrow T_{\xi} Z$ é semi-Fredholm à esquerda, $e$ (2) $O$ espaço $f^{-1}(\xi)$ é Lindelöf.

Então a aplicação $(x, y) \longmapsto y: f^{-1}(\xi) \rightarrow Y$ é $\sigma$-própria.

Demonstração. Seja $p=(x, y) \in f^{-1}(\xi)$. A hipótese (1) e o fato de que $f$ é de classe $C^{1}$ em $A$ possibilitam a aplicação do Lema 3.4 para obtermos uma vizinhança $W_{p} \subset \bar{W}_{p} \subset A$ do ponto $p$ tal que $(a, b) \longmapsto b: \bar{W}_{p} \cap f^{-1}(\xi) \rightarrow Y$ é própria. A hipótese (2), por sua vez, garante que podemos extrair da cobertura $\left\{W_{p} \cap f^{-1}(\xi)\right\}_{p \in f^{-1}(\xi)}$ uma subcobertura enumerável, digamos $\left\{W_{p_{n}} \cap f^{-1}(\xi)\right\}$. Assim é possível escrever $f^{-1}(\xi)=\cup_{n \geq 0} \bar{W}_{p_{n}} \cap f^{-1}(\xi)$, reunião em que $(a, b) \longmapsto b: \bar{W}_{p_{n}} \cap f^{-1}(\xi) \longrightarrow Y$ é aplicação própria para cada $n$.

Lema 3.6. Sejam $k$ um inteiro positivo, $X, Y, Z$ variedades de Banach de classe $C^{k}, f$ uma aplicação de classe $C^{k}$ de um aberto $A \subset X \times Y$ em $Z$ e $\xi$ um ponto de $Z$. Suponha que para $\left(x_{0}, y_{0}\right) \in f^{-1}(\xi)$ tenhamos:

(1) $\frac{\partial f}{\partial x}\left(x_{0}, y_{0}\right): T_{x_{0}} X \longrightarrow T_{\xi} Z$ é um operador semi-Fredholm de indice $<k$.

(2) Uma das seguintes alternativas é verdadeira:

(a) $D f\left(x_{0}, y_{0}\right): T_{x_{0}} \times T_{y_{0}} \longrightarrow T_{\xi} Z$ é sobrejetora, ou 
(b) $\operatorname{dim}\left\{\mathcal{R}\left(D f\left(x_{0}, y_{0}\right)\right) / \mathcal{R}\left(\frac{\partial f}{\partial x}\left(x_{0}, y_{0}\right)\right)\right\} \geq 1+\operatorname{dim} \mathcal{N}\left(\frac{\partial f}{\partial x}\left(x_{0}, y_{0}\right)\right)$.

Entäo existem vizinhanças abertas $U$ de $x_{0}, V$ de $y_{0}$ e um subconjunto aberto $V^{0} \subset V$, denso em $V$, tais que $\bar{U} \times \bar{V} \subset A$ e $\xi$ é um valor regular de $\left.f(\cdot, y)\right|_{U}: U \longrightarrow Z$ para todo $y \in V^{0}$.

Demonstração. Novamente o resultado é local - nas proximidades de $\left(x_{0}, y_{0}\right) \in X \times Y$ e $\xi \in Z$ - e não há perda de generalidade em considerar $X, Y, Z$ espaços de Banach, $x_{0}=0$, $y_{0}=0, \xi=0$ e $f$ de classe $C^{k}$ em uma vizinhança de $(0,0) \in X \times Y$. Para $(x, y)$ na vizinhança acima podemos escrever

$$
f(x, y)=L x+M y+o(\|x\|+\|y\|)
$$

onde $\left.M=\frac{\partial f}{\partial y}\left(x_{0}, y_{0}\right)\right)$ e $\left.L=\frac{\partial f}{\partial x}\left(x_{0}, y_{0}\right)\right)$ é um operador semi-Fredholm com índice $<\mathrm{k}$.

Segundo a primeira observação que fizemos a respeito do enunciado do Teorema da Transversalidade, se a hipótese (2)(a) estiver satisfeita com ind $L<0$ então também o estará a hipótese (2)(b). Por esta razão é suficiente demonstrar o resultado no caso (a) apenas quando ind $L \geq 0$ e no caso (b), quando necessariamente ind $L<0$.

Caso (a): Iremos supor que $\mathcal{R}(L, M)=Z$ e ind $L \geq 0$. Como ind $L$ é finito, $L$ é Fredholm. Desse modo, se $X_{1}=\mathcal{N}(L)$ e $Z_{2}=\mathcal{R}(L)$, existem subespaços fechados $X_{2} \subset X$ e $Z_{1} \subset Z$ tais que $X=X_{1} \oplus X_{2}, Z=Z_{1} \oplus Z_{2}$, o operador $L_{2}=L_{X_{2}}: X_{2} \rightarrow Z_{2}$ é um isomorfismo e $\operatorname{ind} L=\operatorname{dim} X_{1}-\operatorname{dim} Z_{1}$. Observamos que o fato de termos $Z=Z_{2} \oplus \mathcal{R}(M)$ permite a suposição adicional de que $Z_{1} \subset \mathcal{R}(M)$.

Seja $Y_{1}$ um subsepaço de $Y$ para o qual $M_{1}=\left.M\right|_{Y_{1}}: Y_{1} \longrightarrow Z_{1}$ é um isomorfismo e defina $Y_{2}=M^{-1}\left(Z_{2}\right)$. Então $Y=Y_{1} \oplus Y_{2}$. Escrevendo $f$ em termos de suas componentes nestes espaços, temos

$$
\begin{aligned}
f\left(x_{1}, x_{2}, y_{1}, y_{2}\right) & =\left(M_{1} y_{1}+o_{1}(\|x\|+\|y\|), L_{2} x_{2}+M y_{2}+o_{2}(\|x\|+\|y\|)\right. \\
& =\left(M_{1} y_{1}+g\left(x_{1}, x_{2}, y_{1}, y_{2}\right), L_{2} x_{2}+h\left(x_{1}, x_{2}, y_{1}, y_{2}\right)\right)
\end{aligned}
$$

onde $g$ e $h$ são aplicações de classe $C^{k}, g, h, g_{x}, g_{y}$ e $h_{x}$ se anulam todas em $(0,0)$, embora talvez possamos ter $h_{y} \neq 0$, dependendo do comportamento de $M$ no subespaço $Y_{2}$. Notando que $\frac{\partial f}{\partial\left(x_{2}, y_{1}\right)}(0,0,0,0)=\left(L_{2}, M_{1}\right)$ é um isomofismo, utilizamos o teorema da função implícita para resolver a equação $f(x, y)=0$ para $x_{2}=\psi\left(x_{1}, y_{2}\right)$ e $y_{1}=\phi\left(x_{1}, y_{2}\right)$, com $\psi$ e $\phi$ funções de classe $C^{k}$ em uma vizinhança de $(0,0) \in X_{1} \times Y_{2}$. Podemos então selecionar uma vizinhança $W$ de $(0,0) \in X \times Y$ com a seguinte propriedadc: se $\left(x_{1}, x_{2}, y_{1}, y_{2}\right) \in W$ e $f\left(x_{1}, x_{2}, y_{1}, y_{2}\right)=0$ então $x_{2}=\psi\left(x_{1}, y_{2}\right)$ e $y_{1}=\phi\left(x_{1}, y_{2}\right)$. 
Na forma matricial $\frac{\partial f}{\partial x}(x, y)$ se escreve como

$$
\frac{\partial f}{\partial x}(x, y)=\left(\begin{array}{ll}
g_{x_{1}}(x, y) & g_{x_{2}}(x, y) \\
h_{x_{1}}(x, y) & h_{x_{2}}(x, y)
\end{array}\right)
$$

e pode ser reescrita como o produto

$$
\left(\begin{array}{ll}
g_{x_{1}}(x, y) & g_{x_{2}}(x, y) \\
h_{x_{1}}(x, y) & h_{x_{2}}(x, y)
\end{array}\right)=\left(\begin{array}{cc}
I_{Z_{1}} & p(x, y) \\
0 & I_{Z_{2}}
\end{array}\right)\left(\begin{array}{cc}
\Delta(x, y) & 0 \\
0 & L_{2}+h_{x_{2}}(x, y)
\end{array}\right)\left(\begin{array}{cc}
I_{X_{1}} & 0 \\
q(x, y) & I_{X_{2}}
\end{array}\right),
$$

no qual

$$
\begin{aligned}
& \Delta(x, y)=g_{x_{1}}(x, y)-g_{x_{2}}(x, y)\left(L_{2}+h_{x_{2}}(x, y)\right)^{-1} h_{x_{1}}(x, y): X_{1} \longrightarrow Z_{1} \\
& q(x, y)=\left(L_{2}+h_{x_{2}}(x, y)\right)^{-1} h_{x_{1}}(x, y): X_{1} \longrightarrow Z_{2} \\
& p(x, y)=g_{x_{2}}(x, y)\left(L_{2}+h_{x_{2}}(x, y)\right)^{-1}: Z_{2} \longrightarrow Z_{1} .
\end{aligned}
$$

para $(x, y)$ em uma vizinhança $W_{1} \subset W$ escolhida pequena o suficiente para que $L_{2}+h_{x_{2}}$ seja inversivel. Como os operadores dados pelas matrizes nas extremidades do produto matricial acima são sobrejetores, resulta que $\frac{\partial f}{\partial x}(x, y)$, para $(x, y) \in W_{1}$, é sobrejetor se, e somente se, $\Delta(x, y)$ é sobrejetor.

Levando em consideraçāo que $f\left(x_{1}, \psi\left(x_{1}, y_{2}\right), \phi\left(x_{1}, y_{2}\right), y_{2}\right)=0$, obtemos as identidades

$$
\left\{\begin{array}{l}
h_{x_{1}}\left(\sigma\left(x_{1}, y_{2}\right)\right)+\left(L_{2}+h_{x_{2}}\left(\sigma\left(x_{1}, y_{2}\right)\right)\right) \psi_{x_{1}}\left(x_{1}, y_{2}\right)+h_{y_{1}}\left(\sigma\left(x_{1}, y_{2}\right)\right) \phi_{x_{1}}\left(x_{1}, y_{2}\right)=0 \\
g_{x_{1}}\left(\sigma\left(x_{1}, y_{2}\right)\right)+g_{x_{2}}\left(\sigma\left(x_{1}, y_{2}\right)\right) \psi_{x_{1}}\left(x_{1}, y_{2}\right)+\left(M_{1}+g_{y_{1}}\left(\sigma\left(x_{1}, y_{2}\right)\right)\right) \phi_{x_{1}}\left(x_{1}, y_{2}\right)=0
\end{array}\right.
$$

em que $\sigma\left(x_{1}, y_{2}\right)=\left(x_{1}, \psi\left(x_{1}, y_{2}\right), \phi\left(x_{1}, y_{2}\right), y_{2}\right)$.

Se estivermos restritos a uma vizinhança de $(0,0) \in X_{1} \times Y_{2}$ pequena o bastante para que $L_{2}+h_{x_{2}}\left(\sigma\left(x_{1}, y_{2}\right)\right)$ seja inversivel, podemos resolver a primeira equação para $\psi_{x_{1}}$ e substituir na primeira, obtendo

$\Delta\left(\sigma\left(x_{1}, y_{2}\right)\right)+\left[M_{1}+g_{y_{1}}\left(\sigma\left(x_{1}, y_{2}\right)\right)-g_{x_{2}}\left(\sigma\left(x_{1}, y_{2}\right)\right)\left(L_{2}+h_{x_{2}}\left(\sigma\left(x_{1}, y_{2}\right)\right)\right)^{-1} h_{y_{1}}\left(\sigma\left(x_{1}, y_{2}\right)\right)\right] \phi_{x_{1}}\left(x_{1}, y_{2}\right)=$ para todo $\left(x_{1}, y_{2}\right)$ em tal vizinhança. Escolha entāo um aberto $W_{1,2}$ contendo $(0,0) \in X_{1} \times$ $Y_{2}$, contido na vizinhança acima, pequeno o bastante para que o coeficiente de $\phi_{x_{1}}$ seja um isomorfismo para todo $\left(x_{1}, y_{2}\right) \in W_{1,2}$.

Tome agora $\epsilon>0$ e $\delta>0$ tais que se $\hat{U}=\left\{x_{1}+x_{2} ; \max \left\{\left\|x_{1}\right\|,\left\|x_{2}\right\|\right\}<\epsilon\right\}$ e $V=$ $\left\{y_{1}+y_{2}: \max \left\{\left\|y_{1}\right\|,\left\|y_{2}\right\|\right\}<\delta\right\}$, então $\tilde{U} \times V \subset W_{1}$ e se $\left(x_{1}+x_{2}, y_{1}+y_{2}\right) \in \bar{U} \times V$ entầo $\left(x_{1}, y_{2}\right) \in W_{1,2}$. Nestas condiçōes, temos que 0 é valor regular de $\left.f\left(\cdot, y_{1}+y_{2}\right)\right|_{\tilde{U}}: \breve{U} \longrightarrow Z$ se, e somente se, $y_{1}$ é valor regular de $\left.\phi\left(\cdot, y_{2}\right)\right|_{B_{X_{1}}(0, \epsilon)}: B_{X_{1}}(0, \epsilon) \longrightarrow Y_{1}$.

Por fim, fixado $y_{2}$ com $\left\|y_{2}\right\|<\delta$, o Teorema de Sard garante a existência de um subconjunto denso $D_{y_{2}}$ de $B_{Y_{1}}(0, \delta)$ tal que todo $y_{1} \in D_{y_{2}}$ é valor regular da aplicação $\phi\left(\cdot, y_{2}\right): B_{X_{1}}(0, \epsilon) \longrightarrow$ $Y_{1}$. Segue que 0 é valor regular de $\left.f\left(\cdot, y_{1}+y_{2}\right)\right|_{\tilde{U}}: \tilde{U} \longrightarrow Z$ sempre que $y_{1} \in D_{y_{2}}$. Definamos pois

$$
V^{0}=\bigcup_{\left\|y_{2}\right\| \leq \delta}\left\{y_{1}+y_{2} ; y_{1} \in D_{y_{2}}\right\} .
$$


Pelo Lema 3.4 existe um aberto $W^{\prime}$ em torno de $\left(x_{0}, y_{0}\right)$ para o qual $(a, b) \longmapsto b: f^{-1}(\xi) \cap$ $\overline{W^{\prime}} \longrightarrow Z$ é uma aplicação própria. Não há perda de generalidade em supor que $\tilde{U} \times V \subset W^{\prime}$. Escolha um aberto $U$ contendo $x_{0}$ tal que $\bar{U} \subset \tilde{U}$. Afirmamos que fixado $\bar{y} \in \tilde{V}^{0}$, existe uma vizinhança $V_{\bar{y}}$ de $\bar{y}$, tal que $V_{\bar{y}} \subset V$, com a a seguinte propriedade: se $y^{\prime} \in V_{\bar{y}}$ então a aplicação $\left.f\left(\cdot, y^{\prime}\right)\right|_{U}: U \rightarrow Z$ tem o zero como valor regular.

De fato, suponha que não exista uma tal vizinhança. Então existem sequências $\left\{y_{n}\right\}$ em $V$ e $\left\{x_{n}\right\}$ cm $U$, com $y_{n} \rightarrow y$, tais que $f\left(x_{n}, y_{n}\right)=0$ e $\frac{\partial f}{\partial x}\left(x_{n}, y_{n}\right)$ não é sobrejetora para todo $n$. Como $\left(x_{n}, y_{n}\right) \in f^{-1}(0) \cap \overline{W^{\prime}}$ para todo $n$ e $y_{n} \rightarrow y$, a sequência $\left\{x_{n}\right\}$ admite uma subsequência convergente $\left\{x_{n_{k}}\right\}$. Se $\bar{x}=\lim x_{n_{k}}$ temos que $\bar{x} \in \tilde{U}, f(\bar{x}, \bar{y})=0$ e $\frac{\partial f}{\partial x}(\bar{x}, \bar{y})$ não é sobrejetora, o que contraria o fato de que $\left.f(\cdot, y)\right|_{\bar{U}}: \tilde{U} \rightarrow Z$ tem o zero como valor regular para todo $y \in \overline{V^{0}}$.

Assim, tomando

$$
V^{0}=\bigcup_{\bar{y} \in \bar{V}^{0}} V_{\bar{y}},
$$

temos que $V^{0}$ é aberto e denso em $V$ (pois $\tilde{V}^{0}$ é claramente denso em $V$ ) e, além disso, zero é valor regular de $\left.f(\cdot, y)\right|_{U}: U \longrightarrow Z$ para todo $y \in V^{0}$.

Caso (b): Aqui, $\operatorname{dim}\{\mathcal{R}(L, M) / \mathcal{R}(L)\}>\operatorname{dim} \mathcal{N}(L)$ e ind $L<0$. Sejam $n=\operatorname{dim} \mathcal{N}(L)$ e $V \subset \mathcal{R}(M)$ tal que $\mathcal{R}(L, M)=\mathcal{R}(L) \oplus V$. Então $\operatorname{dim} V \geq n+1$ e existe subconjunto $\left\{f_{1}, \ldots, f_{n+1}\right\} \subset V$ linearmente independente. Se $f_{i}=M y_{i}$, para $i=1, \ldots, n+1$, o conjunto $\left\{y_{1}, \ldots, y_{n+1}\right\}$ é linearmente independente (caso contrário $\left\{f_{1}, \ldots, f_{n+1}\right\}$ não o seria). Tome $Y_{1}=\left[\left\{y_{1}, \ldots, y_{n+1}\right\}\right]$. Note que $M\left(Y_{1}\right) \cap \mathcal{R}(L)=\{0\}$ e que $M$ é injetora sobre $Y_{1}$, o que se deve novamente ao fato de $\left\{f_{1}, \ldots, f_{n+1}\right\}$ ser linearmente independente. Sejam $Z_{1}=M\left(Y_{1}\right)$ e $Z_{2} \subset Z$ um subespaço tal que $\mathcal{R}(L) \subset Z_{2}$ e $Z=Z_{1} \oplus Z_{2}$. Tome $Y_{2}=M^{-1}\left(Z_{2}\right)$, de modo que $Y=Y_{1} \oplus Y_{2}$. Sejam, por fim, $X_{1}=\mathcal{N}(L)$ e $X_{2} \subset X$ tal que $X=X_{1} \oplus X_{2}$. Temos que $n=\operatorname{dim} X_{1}<\operatorname{dim} Z_{1}=\operatorname{dim} Y_{1}$.

Nestas circunstâncias $f$ assume a forma

$$
\begin{aligned}
f\left(x_{1}, x_{2}, y_{1}, y_{2}\right) & =\left(M_{1} y_{1}+\mathrm{o}_{1}(\|x\|+\|y\|), L_{2} x_{2}+M y_{2}+\mathrm{o}_{2}(\|x\|+\|y\|)\right) \\
& =\left(M_{1} y_{1}+g\left(x_{1}, x_{2}, y_{1}, y_{2}\right), L_{2} x_{2}+h\left(x_{1}, x_{2}, y_{1}, y_{2}\right)\right),
\end{aligned}
$$

onde $M_{1}=\left.M\right|_{Y_{1}}: Y_{1} \longrightarrow Z_{1}$ é um isomorfismo e $\left.L_{2}\right|_{X_{2}}: X_{2} \longrightarrow Z_{2}$ é injetora com imagem fechada. Além disso, $g$ e $h$ são aplicaçōes de classe $C^{k}$ em uma vizinhança de $(0,0) \in X \times Y$ e $g, g_{x}, g_{y}, h, h_{x}$ se anulam no ponto $(0,0)$. Definindo $\Phi\left(x_{1}, x_{2}, y_{1}, y_{2}\right)=M_{1} y_{1}+g\left(x_{1}, x_{2}, y_{1}, y_{2}\right)$, observamos que $\frac{\partial \Phi}{\partial y_{1}}(0,0,0,0)=M_{1}$ é um isomorfismo e $\Phi(0,0,0,0)=0$. Empregando o teorema da função implícita solucionamos $M_{1} y_{1}+g\left(x_{1}, x_{2}, y_{1}, y_{2}\right)=0$ para $y_{1}=\psi\left(x_{1}, x_{2}, y_{2}\right)$ aplicação de classe $C^{k}$ em uma vizinhança de $(0,0,0) \in X_{1} \times X_{2} \times Y_{2}$, com $\psi_{x}(0,0,0)=0$. Seja $W$ uma vizinhança de $(0,0) \in X \times Y$ com a propriedade de que se $\left(x_{1}, x_{2}, y_{1}, y_{2}\right) \in W$ e $\Phi\left(x_{1}, x_{2}, y_{1}, y_{2}\right)=$ 0 então $y_{1}=\psi\left(x_{1}, x_{2}, y_{2}\right)$. 
Escolha agora $\delta>0$ de modo que tenhamos $\left\{\left(x_{1}+x_{2}, y_{1}+y_{2}\right) ; \max \left\{\left\|x_{1}\right\|,\left\|x_{2}\right\|\right\}<\delta\right.$ e $\max \left\{\left\|y_{1}\right\|\right.$, $\left.\left.\left\|y_{2}\right\|\right\}<\delta\right\} \subset W$. Fixe $y_{2} \operatorname{com}\left\|y_{2}\right\|<\delta$ e seja

$$
S\left(y_{2}\right)=\left\{x=\left(x_{1}, x_{2}\right) ;\left\|x_{1}\right\|<\delta,\left\|x_{2}\right\|<\delta, f\left(x_{1}, x_{2}, \psi\left(x_{1}, x_{2}, y_{2}\right), y_{2}\right)=0\right\}
$$

Sejam ainda $p_{1}: X_{1} \times X_{2} \longrightarrow X_{1}$ a projeção sobre $X_{1}$ e $\pi\left(y_{2}\right)=\left.p_{1}\right|_{S\left(y_{2}\right)}: S\left(y_{2}\right) \longrightarrow X_{1}$. Se $\delta>0$ é suficientemente pequeno, $\pi\left(y_{2}\right)$ é injetora com inversa lipschitziana. Assumindo isto obtemos que

$$
\psi\left(S\left(y_{2}\right), y_{2}\right)=\psi\left(\cdot, y_{2}\right) \circ\left(\pi\left(y_{2}\right)\right)^{-1}\left(\pi\left(y_{2}\right)\left(S\left(y_{2}\right)\right)\right) \subset Y_{1}
$$

é a imagem lipschitziana de um conjunto em $X_{1}$. Como $\operatorname{dim} X_{1}<\operatorname{dim} Y_{1}$, chegamos à conclusão de que $\psi\left(S\left(y_{2}\right), y_{2}\right)$ tem medida nula em $Y_{1}$, do que resulta ser $\psi\left(S\left(y_{2}\right), y_{2}\right)$ magro em $Y_{1}$. Portanto o conjunto $D_{y_{2}}=\left\{y_{1} \in Y_{1} ;\left\|y_{1}\right\|<\delta\right.$ e $\left.y_{1} \notin \psi\left(S\left(y_{2}\right), y_{2}\right)\right\}$ é denso em $B_{Y_{1}}(0, \delta)$. Se $\tilde{U}=\left\{x_{1}+x_{2} ; \max \left\{\left\|x_{1}\right\|,\left\|x_{2}\right\|\right\}<\delta\right\}$, notamos que 0 é valor regular de $\left.f\left(\cdot, y_{1}+y_{2}\right)\right|_{\tilde{U}}: \tilde{U} \rightarrow Z$ para todo $y_{1} \in D_{y_{2}}$ pois 0 não é atingido.

Considere então $V=\left\{y_{1}+y_{2} ; \max \left\{\left\|y_{1}\right\|,\left\|y_{2}\right\|\right\}<\delta\right\} \mathrm{e}$

$$
\tilde{V^{0}}=\bigcup_{\left\|y_{2}\right\|<\delta}\left\{y_{1}+y_{2} ; y_{1} \in D_{y_{2}}\right\}
$$

Escolhendo um aberto $U$ contendo $x_{0}, \operatorname{com} \bar{U} \subset \bar{U}$, usamos raciocínio análogo ao que desenvolvemos no caso (a) para mostrar que para cada $\bar{y} \in \tilde{V^{0}}$ existe um aberto $V_{\bar{y}} \subset V$, contendo $\bar{y}$, tal que zero é valor regular de $\left.f\left(\cdot, y^{\prime}\right)\right|_{U}: U \rightarrow Z$ sempre que $y^{\prime} \in V_{\bar{y}}$. Definindo

$$
V^{0}=\bigcup_{\bar{y} \in V^{0}} V_{\bar{y}}
$$

terminamos a demonstração.

Provemos agora nossa afirmação de que $\pi\left(y_{2}\right)$ tem inversa lipschitziana para $\delta>0$ convenientemente escolhido. Sabemos que $\left\|L x_{2}\right\| \geq c\left\|x_{2}\right\|, x_{2} \in X_{2}$ para alguma constante $c>0$. Uma vez que $\psi_{x}(0,0,0)=0$, tomando $\delta>0$ suficientemente pequeno, temos

$$
\left\|f\left(x, \psi\left(x, y_{2}\right), y_{2}\right)-f\left(\bar{x}, \psi\left(\bar{x}, y_{2}\right), y_{2}\right)-L(x-\bar{x})\right\| \leq \frac{c}{2}\|x-\bar{x}\|
$$

para $\|x\|<\delta,\|\bar{x}\|<\delta$ e $\left\|y_{2}\right\|<\delta$.

Se, adicionalmente, $x, \tilde{x} \in S\left(y_{2}\right)$ obtemos

$$
\begin{aligned}
c\left\|x_{2}-\bar{x}_{2}\right\| & \leq\left\|L\left(x_{2}-\bar{x}_{2}\right)\right\|=\|L(x-\bar{x})\| \leq \frac{c}{2}\|x-\tilde{x}\| \\
& \leq \frac{c}{2}\left\|x_{1}-\bar{x}_{1}\right\|+\frac{c}{2}\left\|x_{2}-\bar{x}_{2}\right\|,
\end{aligned}
$$

de modo que

$$
\left\|x_{2}-\bar{x}_{2}\right\| \leq\left\|x_{1}-\bar{x}_{1}\right\|=\left\|\pi\left(y_{2}\right)(x)-\pi\left(y_{2}\right)(\bar{x})\right\|,
$$


ou seja,

$$
\left\|\pi\left(y_{2}\right)(x)-\pi\left(y_{2}\right)(\bar{x})\right\| \geq \frac{1}{2}\|x-\bar{x}\|
$$

o que completa a demonstração.

Demonstração do Teorema da Transversalidade. Admitiremos inicialmente que $(x, y) \longmapsto$ $y: f^{-1}(\xi) \longrightarrow Y$ é própria e provaremos que $Y_{\text {crit }}$ é fechado com interior vazio.

Para verificarmos que $Y_{\text {crit }}$ é fechado seja $\left\{y_{n}\right\}$ uma sequência em $Y_{\text {crit }}$ que converge em $Y$ para um ponto $\bar{y}$. Para cada inteiro positivo $n$ existe $x_{n} \in A_{y_{n}}$ ponto crítico de $f\left(\cdot, y_{n}\right)$ : $A_{y_{n}} \longrightarrow Z$ tal que $f\left(x_{n}, y_{n}\right)=\xi$. A suposição de que $(x, y) \longmapsto y: f^{-1}(\xi) \longrightarrow Y$ é própria implica a existência de uma subsequência convergente $\left\{\left(x_{n_{k}}, y_{n_{k}}\right)\right\}$, convergindo, digamos, para $(\bar{x}, \bar{y}) \in f^{-1}(\xi)$. Se o operador $\frac{\partial f}{\partial x}(\bar{x}, \bar{y})$ fosse sobrejetor, concluiríamos que para $n$ suficientemente grande os operadores $\frac{\partial f}{\partial x}\left(x_{n}, y_{n}\right)$ são sobrejetores, uma contradição. Segue que $\bar{x}$ é ponto crítico de $f(\cdot, \bar{y}): A_{\bar{y}} \longrightarrow Z$, com $f(\bar{x}, \bar{y})=\xi$, isto é, $\bar{y} \in Y_{\text {crit }}$.

Resta ainda demonstrar que $Y_{c r i t}$ tem interior vazio, ou seja, precisamos verificar que dado $y \in Y_{\text {crit }}$ existe $y^{\prime}$ arbitrariamente próximo de $y$ tal que $\xi$ é valor regular de $f\left(\cdot, y^{\prime}\right): A_{y^{\prime}} \longrightarrow Z$. Para isto, tome $y \in Y_{c r i t}$ e seja $K_{y}=\{x ; f(x, y)=\xi\}$. Da hipótese de que $(x, y) \longmapsto y$ : $f^{-1}(\xi) \longrightarrow Y$ é própria segue que $K_{y}$ é compacto.

Para cada $x \in K_{y}$ podemos aplicar o Lema 3.6 para o ponto $(x, y)$, obtendo abertos $U_{x} \subset X$, $V_{x} \subset Y$ e $V_{x}^{0} \subset V_{x}$ tais que $x \in U_{x}, y \in V_{x}, V_{x}^{0}$ é denso em $V_{x}, \bar{U}_{x} \times \bar{V}_{x} \subset A$ e $f\left(\cdot, y^{\prime}\right): U_{x} \longrightarrow Z$ tem $\xi$ como valor regular para todo $y^{\prime} \in V_{x}^{0}$.

A compacidade de $K_{y}$ garante a existência de $x_{1}, \ldots, x_{N} \in K_{y}$ tais que $K_{y} \subset U_{x_{1}}, \ldots, U_{x_{N}}$. $\operatorname{Sejam} \tilde{U}=U_{x_{1}} \cup \cdots \cup U_{x_{N}}, \tilde{V}=V_{x_{1}} \cap \cdots \cap V_{x_{N}}$ e $\tilde{V}^{0}=V_{x_{1}}^{0} \cap \cdots \cap V_{x_{N}}^{0}$. É fácil ver que $\tilde{V}^{0}$ é denso em $V$ e que $\xi$ é valor regular de $\left.f(\cdot, \tilde{y})\right|_{\bar{U}}: \tilde{U} \longrightarrow Z$ para todo $\tilde{y} \in \tilde{V}^{0}$.

Afirmamos que, na verdade, $\xi$ é valor regular de $f(\cdot, \tilde{y}): A_{\bar{y}} \longrightarrow Z$ para todo $\bar{y} \in \tilde{V}^{0}$ suficientemente próximo de $y$.

Para sermos mais precisos, seja $\mathcal{B}=\left\{B_{n}\right\}$ uma base enumerável de vizinhanças em y para a topologia de $Y$ e, para cada, $n$ considere $W_{n}=\cap_{i=1}^{n} B_{i}$. Claramente os $W_{n}$ são abertos com $W_{1} \supset W_{2} \supset W_{3} \supset \cdots$

Suponha, para chegar a um absurdo, que tenhamos $\left(W_{n} \cap \tilde{V}^{0}\right) \cap Y_{\text {crit }} \neq \varnothing$ para cada $n$. Então existe uma sequência $\left\{y_{n}\right\}$ em $\tilde{V}^{0}$ tal que $y_{n} \longrightarrow y$ e $\xi$ é valor crítico de $f\left(\cdot, y_{n}\right): A_{y_{n}} \longrightarrow Z$ para todo $n$. Desse modo, para cada $n$ existe $x_{n} \in A_{y_{n}}$ ponto crítico de $f\left(\cdot, y_{n}\right): A_{y_{n}} \longrightarrow Z$, com $f\left(x_{n}, y_{n}\right)=\xi$. Usando urna vez mais o fato de que $(x, y) \longmapsto y: f^{-1} \longrightarrow Y$ é própria, podemos supor que $x_{n} \longrightarrow x$ em $X$ (caso contrário passamos a uma subsequência convergente). Agora $f(x, y)=\xi$, de maneira que $x \in K_{y}$. Logo, para $n$ suficientemente grande concluímos que $x_{n} \in U, y_{n} \in \tilde{V}^{0}$ e chegamos a uma contradição. 
Portanto, existe $N_{0}$ tal que $\left(W_{N_{0}} \cap \tilde{V}^{0}\right) \cap Y_{\text {crit }}=\varnothing$ e por conseguinte $\left(W_{n} \cap \tilde{V}^{0}\right) \cap Y_{\text {crit }}=\varnothing$ para todo $n \geq N_{0}$.

Finalmente, seja $W$ um aberto de $Y$ que contém $y$. Escolha $\bar{N}>N_{0}$ tal que $y \in W_{\bar{N}} \subset W$. Então $\tilde{V} \cap W_{\bar{N}}$ é vizinhança de $y$ em $\tilde{V}$. Pela densidade de $\tilde{V}^{0}$ em $\tilde{V}$ existe $p \in \tilde{V} \cap W_{\tilde{N}} \cap \tilde{V}^{0}$. Como $\bar{N}>N_{0}$, temos $\left(W_{\bar{N}} \cap \tilde{V}^{0}\right) \cap Y_{c r i t}=\varnothing$ e, necessariamente, $p \notin Y_{\text {crit }}$. Segue que $p \in W \cap Y_{\text {crit }}^{c}$. Com isto finalizamos a demonstração de que $Y_{\text {crit }}$ tem interior vazio.

Por fim, suponha que $(x, y) \longmapsto y: f^{-1}(\xi) \longrightarrow Y$, ao invés de própria, seja apenas $\sigma$-própria. Neste caso, $f^{-1}(\xi)=\cup_{j=1}^{\infty} M_{j}$ e $(x, y) \longmapsto y: M_{j} \longrightarrow Y$ é própria para todo $j$. Defina

$Y_{\text {crit }}^{j}=\left\{y\right.$; existe um ponto crítico $x$ de $f(\cdot, y): A_{y} \rightarrow Z$ tal que $f(x, y)=\xi$ e $\left.(x, y) \in M_{j}\right\}$.

É imediata a conclusão de que $Y_{\text {crit }}=\cup_{j=1}^{\infty} Y_{\text {crit }}^{j}$. Repetindo o argumento que utilizamos acima, demonstramos que $Y_{\text {crit }}^{j}$ é fechado e tem interior vazio para cada $j$, obtendo que $Y_{\text {crit }}$ é subconjunto magro de $Y$.

Sob hipóteses um pouco mais restritivas é possível obter informações mais precisas, em algum sentido, sobre o conjunto $Y_{\text {crit }}$. É disso que trata o Corolário 3.7 que provaremos a seguir. Antes, porém, precisamos de algumas definições.

Dizemos que um espaço topológico $X$ é um espaço de Baire se todo subconjunto magro de $X$ tem interior vazio. É nesses espaços que a noção de subconjunto magro realmente destaca uma classe de conjuntos que são insignificantes diante do espaço todo.

Seja $E$ um espaço topológico de Baire. Para qualquer subconjunto fechado ou $\sigma$-fechado $F \subset E$ e qualquer inteiro $m \geq 0, \operatorname{codim} F>m$ significa que o conjunto

$$
\left\{\phi \in C\left(S^{m}, E\right) ; \phi\left(S^{m}\right) \cap F \neq \emptyset\right\}
$$

é magro em $C\left(S^{m}, E\right)$. Aqui, $S^{m}=\partial \overline{B^{m+1}}$, onde $B^{m+1}$ é a bola unitária em $\mathbb{R}^{m+1}$, para $m>0$, $S^{0}=\{0\}$ e $C\left(S^{m}, E\right)$ está munido da topologia compacto-aberta.

Analogamente, se $E$ é um espaço de Banach ou uma variedade de Banach de classe $C^{1}$ dizemos que $C^{1}$ - $\operatorname{codim} F>m$ se o conjunto

$$
\left\{\phi \in C^{1}\left(S^{m}, E\right) ; \phi\left(S^{m}\right) \cap F \neq \varnothing\right\}
$$

é magro em $C^{1}\left(S^{m}, E\right)$.

Prova-se que se $\operatorname{codim} F>m$ para algum $m>0$ então $\operatorname{codim} F>m-1$. Isto nos permite definir a codimensão e não somente a frase "codim $F>m$ ".

Assim, se $E$ é um espaço de Baire e $F \subset E$ é um subconjunto fechado ou $\sigma$-fechado, afirmamos que $\operatorname{codim} F=m$ quando $\operatorname{codim} F \ngtr m$ e $m=0$ ou $\operatorname{codim} F>m-1$. Assim, para 
cada subconjunto fechado ou $\sigma$-fechado $F$ existe $m \in\{0,1,2, \ldots, \infty\}$ tal que $\operatorname{codim} F=m$ e chamamos $m$ de codimensão de $F$.

Corolário 3.7. Sejam $k$ e $m$ inteiros positivos, $X, Y$ e $Z$ variedades de Banach de classe $C^{k}$, $A \subset X \times Y$ aberto, $f: A \longrightarrow Z$ aplicação de classe $C^{k}$ e $\xi \in Z$. Suponha que para todo $(x, y) \in f^{-1}(\xi)$ tenhamos:

(1) $\frac{\partial f}{\partial x}(x, y): T_{x} X \longrightarrow T_{\xi} Z$ é semi-Fredholm com índice $\leq-m$.

(2) Uma das seguintes alternativas é válida:

(a) $D f(x, y)=\left(\frac{\partial f}{\partial x}(x, y), \frac{\partial f}{\partial y}(x, y)\right): T_{x} X \times T_{y} Y \rightarrow T_{\xi} Z$ é sobrejetora, ou

(b) $\operatorname{dim}\left\{\mathcal{R}(D f(x, y)) / \mathcal{R}\left(\frac{\partial f}{\partial x}(x, y)\right)\right\} \geq m+\operatorname{dim} \mathcal{N}\left(\frac{\partial f}{\partial x}(x, y)\right)$.

Suponha ainda que

(3) $(x, y) \longmapsto y: f^{-1}(\xi) \longrightarrow Y$ é uma aplicação $\sigma$-própria, ou seja, $f^{-1}(\xi)=\cup_{j=1}^{\infty} M_{j}$ é uma reunião enumerável de conjuntos $M_{j}$ tais que $(x, y) \longmapsto y: M_{j} \rightarrow y$ é uma aplicação própria para cada $j$.

Sejam $A_{y}=\{x \in X ;(x, y) \in A\} e$

$$
Y_{\text {crit }}=\left\{y \in Y ; \xi \text { é um valor crítico de } f(\cdot, y): A_{y} \longrightarrow Z\right\} \text {. }
$$

Então $Y_{\text {crit }}=\left\{y ; \xi \in f\left(A_{y}, y\right)\right\}$ tem codimensão $\geq m$ em $Y$.

Demonstração. Primeiramente observamos que a hipótese de que ind $\frac{\partial f}{\partial x}(x, y) \leq-m$ para todo $(x, y) \in f^{-1}(\xi)$ implica que $\operatorname{codim} \mathcal{R}\left(\frac{\partial f}{\partial x}(x, y)\right)>0$ qualquer que seja $(x, y) \in f^{-1}(\xi)$, 。 que é suficiente para concluírmos que $Y_{\text {crit }}=\left\{y ; \xi \in f\left(A_{y}, y\right)\right\}$.

Serão tratados separadamente os casos $m=1$ e $m>1$. No primeiro caso, $m=1$, estão satisfeitas a hipótese (3) e as hipóteses (1) e (2)(b) do Teorema da Transversalidade em todos os pontos de $f^{-1}(\xi)$. Isto garante que

$$
Y_{\text {crit }}=\left\{y ; \xi \in f\left(A_{y}, y\right)\right\}
$$

é magro e tem, portanto, codimensão $\geq 1$.

Para tratarmos o caso $m>1$ reduziremos o problema ao caso $m=1$ da seguinte maneira. Sejam $\tilde{X}=X \times S^{m-1}, \tilde{Y}=C^{k}\left(S^{m-1}, Y\right), \tilde{Z}=Z$ e $\tilde{A}=\{(x, t, \tilde{y}) ;(x, \tilde{y}(t)) \in A\}$. Defina $\tilde{f}: \tilde{A} \subset \tilde{X} \times \tilde{Y} \longrightarrow \tilde{Z}$ por $\tilde{f}(x, t, \tilde{y})=f(x, \tilde{y}(t))$. Veremos que $\tilde{f}$ satifaz as mesmas hipóteses do enunciado, exceto pela substituição de $m$ por 1 .

Sabemos que $f^{-1}(\xi)=\cup_{j=1}^{\infty} M_{j}$ é uma reuniāo enumerável de conjuntos $M_{j}$ tais que $(x, y) \longmapsto$ $y: M_{j} \longrightarrow Y$ é própria para cada $j$. Defina $\tilde{M}_{j}=\left\{(x, t, \tilde{y}) ;(x, \tilde{y}(t)) \in M_{j}\right\}, j=1,2, \ldots$

Fixemos um inteiro positivo arbitrário $j$ e provemos que $(x, t, \tilde{y}) \longmapsto \tilde{y}: \tilde{M}_{j} \longrightarrow \tilde{Y}$ é própria. Seja $\left\{\left(x_{n}, t_{n}, \bar{y}_{n}\right)\right\}$ sequência em $\tilde{M}_{j}$ tal que $\tilde{y}_{n} \rightarrow y$ em $\tilde{Y}$. Como $S^{m-1}$ é compacto, 
podemos supor que $\left\{t_{n}\right\}$ converge para $t \in S^{m-1}$ (se assim não o for basta tomarmos uma subsequência convergente). Logo, $\left\{\left(x_{n}, \tilde{y}_{n}\left(t_{n}\right)\right)\right\}$ é sequência em $M_{j}$ tal que $\tilde{y}_{n}\left(t_{n}\right) \longrightarrow \tilde{y}(t)$. Como $(x, y) \longmapsto y: M_{j} \longrightarrow Y$ é própria, existe subseqência $\left\{x_{n_{k}}, \tilde{y}_{n_{k}}\left(t_{n_{k}}\right)\right\}$ que converge em $M_{j}$, do que segue a convergência de $\left\{\left(x_{n_{k}}, t_{n_{k}}, \tilde{y}_{n_{k}}\right)\right\}$ em $M_{j}$. Portanto, $\tilde{f}$ satisfaz a hipótese (3).

Tome agora $(x, t, \tilde{y}) \in \tilde{f}^{-1}(\xi)$, ou seja, $f(x, \tilde{y}(t))=\xi$. Temos

$$
\frac{\partial \tilde{f}}{\partial(x, t)}(x, t, \tilde{y})(u, s)=\frac{\partial f}{\partial x}(x, \tilde{y}(t)) u+\left(\frac{\partial f}{\partial y}(x, \tilde{y}(t)) \tilde{y}^{\prime}(t)\right) s,
$$

para $(u, s) \in T_{x} X \times T_{t}\left(S^{m-1}\right) \mathrm{e}$

$$
D \tilde{f}(x, t, \tilde{y})(u, s, h)=\frac{\partial f}{\partial x}(x, \tilde{y}(t)) u+\left(\frac{\partial f}{\partial y}(x, \tilde{y}(t)) \tilde{y}^{\prime}(t)\right) s+\frac{\partial f}{\partial y}(x, \tilde{y}(t)) h(t),
$$

para $(u, s, h) \in T_{x} X \times T_{t}\left(S^{m-1}\right) \times C^{k}\left(S^{m-1}, Y\right)$.

Sejam $p$ e $q$ as dimensões dos espaços

$$
\frac{\mathcal{R}\left(\frac{\partial f}{\partial y}(x, \bar{y}(t)) \tilde{y}^{\prime}(t)\right)}{\left(\mathcal{R}\left(\frac{\partial f}{\partial x}(x, \tilde{y}(t))\right) \cap \mathcal{R}\left(\frac{\partial f}{\partial y}(x, \tilde{y}(t)) \tilde{y}^{\prime}(t)\right)\right)}
$$

e

$$
\frac{\mathcal{R}\left(\frac{\partial f}{\partial y}(x, \tilde{y}(t))\right)}{\mathcal{R}\left(\frac{\partial f}{\partial y}(x, \tilde{y}(t))\right) \cap\left(\mathcal{R}\left(\frac{\partial f}{\partial x}(x, \tilde{y}(t))\right)+\mathcal{R}\left(\frac{\partial f}{\partial y}(x, \tilde{y}(t)) \tilde{y}^{\prime}(t)\right)\right)}
$$

respectivamente. Temos

$$
\operatorname{dim} \mathcal{N}\left(\frac{\partial \tilde{f}}{\partial(x, t)}(x, t, \tilde{y})\right)=\operatorname{dim} \mathcal{N}\left(\frac{\partial f}{\partial x}(x, \tilde{y}(t))\right)+(m-1)-q
$$

e

$$
\operatorname{codim} \mathcal{R}\left(\frac{\partial \bar{f}}{\partial(x, t)}(x, t, \tilde{y})\right)=\operatorname{codim} \mathcal{R}\left(\frac{\partial f}{\partial x}(x, \tilde{y}(t))\right)-q
$$

resultando que

$$
\text { ind } \frac{\partial \vec{f}}{\partial(x, t)}(x, t, \tilde{y})=\text { ind } \frac{\partial f}{\partial x}(x, \tilde{y}(t))+(m-1) \leq-1 \text {. }
$$

Além disso, $p+q=\operatorname{dim}\left\{\mathcal{R}(D f(x, \tilde{y}(t))) / \mathcal{R}\left(\frac{\partial f}{\partial x}(x, \tilde{y}(t))\right)\right\}$ e a validade, para a função $f$, da hipótese (2)(b) no ponto $(x, \tilde{y}(t))$ fornece que $p+q \geq m+\operatorname{dim} \mathcal{N}\left(\frac{\partial f}{\partial x}(x, \tilde{y}(t))\right)$. Assim,

$$
\operatorname{dim}\left\{\frac{\mathcal{R}(D \tilde{f}(x, t, \tilde{y}))}{\mathcal{R}\left(\frac{\partial \bar{f}}{\partial(x, t)}(x, t, \tilde{y})\right)}\right\} \geq p \geq 1+\operatorname{dim} \mathcal{N}\left(\frac{\partial \tilde{f}}{\partial(x, t)}(x, t, \tilde{y})\right) .
$$

Acabamos de demonstrar que as hipóteses (1) e (2)(b) também são satisfeitas pela aplicação $\tilde{f}$ com 1 em lugar de $m$. Concluímos então que o conjunto

$$
\left\{\tilde{y} \in C^{k}\left(S^{m-1}, Y\right) ; \xi=f(x, \tilde{y}(t)) \text { para algum }(x, t), \operatorname{com}(x, \tilde{y}(t)) \in A\right\}
$$


é magro em $C^{k}\left(S^{m-1}, Y\right)$, o que significa precisamente que $\left\{\tilde{y} \in C^{k}\left(S^{m-1}, Y\right) ; \tilde{y}\left(S^{m-1}\right) \cap Y_{\text {crit }}\right\}$ é magro. Portanto $Y_{\text {crit }}$ tem $C^{k}$-codimensão $\geq m-1 \mathrm{e}$, conseqüentemente, tem codimensão $\geq m-1$. 


\section{Capítulo 4}

\section{Propriedades Genéricas de Equilíbrios de Equações Parabólicas}

Se $\mathcal{P}$ é uma propriedade dependente de um parâmetro $x \in X$, onde $X$ é um espaço topológico de Baire, dizemos que $\mathcal{P}$ é genérica (em $X$ ) quando se verifica em todos os pontos de $X$, exceto nos pontos de um subconjunto magro de $X$.

Neste capítulo apresentamos dois exemplos, de naturezas distintas, tratando da hiperbolicidade genérica dos equilíbrios de equações parabólicas.

No primeiro deles, que ocupa a Seção 4.1, mostraremos que fixada uma região $\Omega$, limitada e com fronteira suave, todos os equilíbrios (soluções estacionárias) do problema de Dirichlet para a equação de reação-difusão

$$
\begin{aligned}
& u_{t}=\Delta u+f(x, u), t>0, x \in \Omega \\
& u=0, t>0, x \in \partial \Omega
\end{aligned}
$$

são hiperbólicos, qualquer que seja a função $f: \Omega \times \mathbb{R} \longrightarrow \mathbb{R}$ tomada em um espaço conveniente de funçöes, a menos de um subconjunto magro. Assim, a genericidade se refere às funções $f$ neste caso.

No segundo exemplo, apresentado na Seção 4.3, a situação é outra: provaremos que dada uma função bem comportada $f: \mathbb{R}^{n} \times \mathbb{R} \times \mathbb{R}^{n} \rightarrow \mathbb{R}$, todos os equilíbrios do problema de Dirichlet

$$
\begin{aligned}
u_{t} & =\Delta u+f(x, u, \nabla u), t>0, x \in \Omega \\
u & =0, t>0, x \in \partial \Omega
\end{aligned}
$$

são hiperbólicos, qualquer que seja a região $\Omega$ escolhida em uma certa classe de regiões, exceto talvez para $\Omega$ em um subconjunto magro desta classe. Portanto, aqui estabelecemos a genericidade com respeito às regiōes $\Omega$. 
Na Seção 4.2 apresentamos um resultado cuja inserção se justifica não apenas pelo fato de ser empregado no segundo exemplo, mas também por seu valor intrínseco.

\subsection{Simplicidade Genérica das Soluções de $\Delta u+f(x, u)=0$}

Considere o problema de Dirichlet para a equação de reação-difusão

$$
\begin{aligned}
u_{t} & =\Delta u+f(x, u), t>0, x \in \Omega \\
u & =0, t>0, x \in \partial \Omega
\end{aligned}
$$

em que $\Omega \subset \mathbb{R}^{N}$ é um domínio limitado com fronteira suave e $f$ é uma função suficientemente regular em $\Omega \times \mathbb{R}$.

Provaremos nesta seção que a hiperbolicidade dos equilíbrios de (4.1), (4.2) é uma propriedade genérica. Em termos precisos, se $\Omega$ é um domínio limitado de classe $C^{3}$ e $C$ denota o conjunto de todas as funções $f: \Omega \times \mathbb{R} \rightarrow \mathbb{R}$ de classe $C^{k}, k \geq 2$, munido da topologia de Whitney, mostraremos que existe um conjunto residual $\mathfrak{C}^{H} \subset \mathfrak{C}$ tal que para toda função $f \in \mathfrak{C}^{H}$ todos os equilíbrios $u$ de (4.1), (4.2) são hiperbólicos, ou seja, a linearização

$$
w \longmapsto \Delta w+\frac{\partial f}{\partial u}(x, u(x)) w: W^{2, p} \cap W_{0}^{1, p} \longrightarrow L^{p}(\Omega)
$$

de $v \rightarrow \Delta v+f(x, v): W^{2, p} \cap W_{0}^{1, p}(\Omega) \longrightarrow L^{p}(\Omega)$ no ponto $u$ é um isomorfismo. Neste caso também é costume dizer que $u$ é uma solução simples de $\Delta v+f(x, v)=0$. Assim, ora faremos referência a $u$ como um equilíbrio hiperbólico, ora como uma solução simples, indiscriminadamente.

Recordamos que a topologia de Whitney é aquela para a qual a coleção de conjuntos

$$
\left\{g \in \mathfrak{C} ;\left|D^{i} f(x, u)-D^{i} g(x, u)\right|<\delta(u), i=1, \ldots, k, x \in \bar{\Omega}, u \in \mathbb{R}\right\} \text {, }
$$

onde $\delta: \mathbb{R} \rightarrow \mathbb{R}$ é uma funçāo positiva contínua, constitui uma base de vizinhanças para um elemento $f$. A topologia de Whitney faz de $\mathcal{C}$ um espaço de Baire.

Teorema 4.1. Existe um conjunto residual $\mathfrak{C}^{H}$ em $\mathfrak{C}$ tal que para qualquer $f \in \mathfrak{C}^{H}$ todos os equilíbrios de (4.1), (4.2) são hiperbólicos.

Demonstração. Para cada $n=1,2, \ldots$ seja

$$
\mathfrak{C}_{n}^{H}=\left\{f \in \mathfrak{C} ; \text { cada equilíbrio } u \text { de }(4.1),(4.2) \text { com }\|u\|_{L^{\infty}(\Omega)} \leq n \text { é hiperbólico }\right\} \text {. }
$$

Vamos demonstrar que $\mathfrak{C}_{n}^{H}$ é aberto e denso em $\mathfrak{C}$ para cada inteiro positivo $n$, de modo que ao tomar $\mathfrak{C}^{H}=\cap_{1}^{\infty} \mathfrak{C}_{n}^{H}$ obtemos um conjunto residual com a propriedade requerida. Com efeito, fixada $g \in \mathfrak{C}^{H}$, se $u$ é um equilíbrio de (4.1), (4.2), temos que $u \in C^{2}(\Omega)$ e, portanto, 
existe inteiro $m>0$ tal que $\|u\|_{L^{\infty}(\Omega)}<m$. Como $g \in \mathfrak{C}_{m}^{H}$, concluímos que $u$ é um equilíbrio hiperbólico.

Para verificar que $\mathfrak{C}_{n}^{H}$ é aberto, mostraremos que $\mathfrak{C} \backslash \mathfrak{C}_{n}^{H}$ é fechado. Sejam $f_{0} \in \overline{\mathfrak{C} \backslash \mathfrak{C}_{n}^{H}}$ e $\left\{f_{k}\right\}$ uma sequência em $\mathfrak{C} \backslash \mathfrak{C}_{n}^{H}$ convergindo para $f_{0}$ na topologia de Whitney. Em particular, $f_{k} \longrightarrow f_{0}$ e $\frac{\partial f_{k}}{\partial u} \longrightarrow \frac{\partial f_{0}}{\partial u}$ uniformemente no compacto $\bar{\Omega} \times[-n, n]$.

Pela definição de $\mathcal{C}_{n}^{H}$ existe uma sequência $\left\{u_{k}\right\}$ em $W^{2, p} \cap W_{0}^{1, p}(\Omega)$ satisfazendo

$$
\left\|u_{k}\right\|_{L^{\infty}(\Omega)} \leq n \quad \text { e } \quad \Delta u_{k}(x)+f_{k}\left(x, u_{k}(x)\right)=0, x \in \Omega
$$

tal que a linearização de $u \longmapsto \Delta u+f_{k}(x, u): W^{2, p} \cap W_{0}^{1, p}(\Omega) \longrightarrow L^{p}(\Omega)$ no ponto $u_{k}$, para cada $k$, não é um isomorfismo.

Levando em consideração que $u_{k} \in C^{2}(\Omega)$, o que assegura que $\frac{\partial f_{k}}{\partial u}\left(\cdot, u_{k}(\cdot)\right) \in C^{0}(\Omega)$, observamos que $w \mapsto \Delta w+\frac{\partial f_{k}}{\partial u}\left(x, u_{k}(x)\right) w: W_{0}^{2, p} \cap W^{1, p}(\Omega) \longrightarrow L^{p}(\Omega)$ (a linearização de $u \longmapsto \Delta u+f_{k}(x, u)$ no ponto $\left.u_{k}\right)$ é um operador de Fredholm de índice zero.

Portanto, deve também existir uma sequência $\left\{v_{k}\right\}$ em $W^{2, p} \cap W_{0}^{1, p}(\Omega)$ tal que

$$
\left\|v_{k}\right\|_{L^{2}(\Omega)}=1 \text { e } \Delta v_{k}(x)+\frac{\partial f_{k}}{\partial u}\left(x, u_{k}(x)\right) v_{k}(x)=0, x \in \Omega
$$

para todo inteiro positivo $k$.

Afirmamos que a sequência $\left\{u_{k}\right\}$ é limitada em $W^{2, p}(\Omega)$. De fato, podemos supor que para alguma constante $M>0$ tenhamos

$$
\left|f_{k}(x, u)\right| \leq M, \quad(x, u) \in \bar{\Omega} \times[-n, n],
$$

pois se não for este o caso, basta que passemos a uma subsequência $\left\{f_{k_{j}}\right\}$ conveniente.

Assim, para $k=1,2, \ldots$

$$
\left|\Delta u_{k}(x)\right|=\left|f_{k}\left(x, u_{k}(x)\right)\right| \leq M,
$$

qualquer que seja $x \in \Omega$. Usando a limitação de $\Omega$, obtemos que

$$
\left\|\Delta u_{k}\right\|_{L^{p}(\Omega)} \leq M(m(\Omega))^{1 / p}
$$

onde $m(\Omega)$ é a medida do aberto $\Omega$.

Sendo o operador $\Delta: W^{2, p} \cap W_{0}^{1, p}(\Omega) \longrightarrow L^{p}(\Omega)$ um isomorfismo, concluímos que $\left\|u_{k}\right\|_{W^{2, p} \cap W_{0}^{1, p}(\Omega)}$ $\leq M^{\prime}$ para algum $M^{\prime}>0$, do que segue a limitação da sequência $\left\{u_{k}\right\}$ em $W^{2, p}(\Omega)$.

Como $W^{2, p}(\Omega) \hookrightarrow W^{1, p}(\Omega)$, não há perda de generalidade em supor que $\left\{u_{k}\right\}$ converge em $W^{1, p}(\Omega)$ para uma função $u_{0} \in W^{1, p}(\Omega)$, que satisfaz $\left\|u_{0}\right\|_{L^{\infty}(\Omega)} \leq n$.

Agora, para $p>N, W^{1, p}(\Omega) \hookrightarrow C^{0}(\bar{\Omega})$, o que nos possibilita supor ainda que $u_{k} \longrightarrow u_{0}$ em $C(\bar{\Omega})$. Este fato, juntamente com a continuidade uniforme de $f_{0}$ no compacto $\bar{\Omega} \times[-n, n]$, a 
convergência uniforme da sequência $\left\{f_{k}\right\}$ para $f_{0}$ em $\bar{\Omega} \times[-n, n]$ e a limitação de $\Omega$ nos levam facilmente a concluir que $f_{k}\left(\cdot, u_{k}(\cdot)\right) \longrightarrow f_{0}\left(\cdot, u_{0}(\cdot)\right)$ em $L^{p}(\Omega)$.

Usando uma vez mais que $\Delta: W^{2, p} \cap W_{0}^{\mathrm{I}, p}(\Omega) \rightarrow L^{p}(\Omega)$ é um isomorfismo, obtemos que

$$
\Delta^{-1}\left(f_{k}\left(\cdot, u_{k}(\cdot)\right)\right) \rightarrow \Delta^{-1}\left(f_{0}\left(\cdot, u_{0}(\cdot)\right)\right) \text { em } W^{2, p} \cap W_{0}^{1, p}(\Omega)
$$

Mas para todo $k$,

$$
u_{k}=-\Delta^{-1}\left(f_{k}\left(\cdot, u_{k}(\cdot)\right)\right)
$$

resultando que

$$
u_{k} \longrightarrow-\Delta^{-1}\left(f_{0}\left(\cdot, u_{0}(\cdot)\right)\right) \text { em } W^{2, p} \cap W_{0}^{\mathrm{I}, p}(\Omega)
$$

Já tínhamos que $u_{k} \longrightarrow u_{0}$ em $W^{1, p}(\Omega)$ e, portanto, $u_{0}=\Delta^{-1}\left(f_{0}\left(\cdot, u_{0}(\cdot)\right)\right) \in W^{2, p} \cap W_{0}^{1, p}(\Omega)$.

Finalmente, como $\Delta u_{k} \longrightarrow \Delta u_{o}$ em $L^{p}(\Omega)$ (pois $u_{k} \longrightarrow u_{0}$ em $W^{2, p} \cap W_{0}^{1, p}(\Omega)$ ) e $f_{k}\left(\cdot, u_{k}(\cdot)\right) \longrightarrow$ $f_{0}\left(\cdot, u_{0}(\cdot)\right)$ em $L^{p}(\Omega)$, vemos que $\Delta u_{k}+f_{k}\left(\cdot, u_{k}(\cdot)\right) \rightarrow \Delta u_{0}+f_{0}\left(\cdot, u_{0}(\cdot)\right)$ em $L^{p}(\Omega)$, o que implica que $\Delta u_{0}(x)+f_{0}\left(x, u_{0}(x)\right)=0, x \in \Omega$, ou seja, $u_{0}$ é um equilíbrio de (4.1), (4.2), quando consideramos $f_{0}$.

Raciocínio inteiramente análogo, aplicado à sequência $\left\{v_{k}\right\}$, fornece que $\left\{v_{k}\right\}$ converge em $W^{2, p} \cap W_{0}^{1, p}(\Omega)$ para uma função $v_{0}$ que satisfaz $\left\|v_{0}\right\|_{L^{2}(\Omega)}=1$ e $\Delta v_{0}(x)+\frac{\partial f_{0}}{\partial u}\left(x, u_{0}(x)\right) v_{0}(x)=$ $0, x \in \Omega$, o que significa que $u_{0}$ não é um equilíbrio hiperbólico, embora tenhamos $\left\|u_{0}\right\|_{L^{\infty}(\Omega)} \leq n$. Provamos pois que $f_{0} \in \mathfrak{C} \backslash \mathfrak{C}_{n}^{H}$.

Vejamos agora que $\mathfrak{C}_{n}^{H}$ é denso. Para isto, fixe $f \in \mathfrak{C}$ e escolha uma função auxiliar $\eta: \mathbb{R} \longrightarrow$ $\mathbb{R}$, de classe $C^{\infty}$, com suporte compacto, que é idêntica a 1 no intervalo $[-n-1, n+1]$.

Vamos verificar que existe um conjunto denso de funções $b$ em $C^{k}(\Omega)$ para as quais a função $f(x, u)+b(x) \eta(u)$ pertence a $\mathfrak{C}_{n}^{H}$. É claro que esta é uma função arbitrariamente próxima de $f$ em $\mathfrak{C}$ desde que $b$ esteja suficientemente próxima de 0 , do que decorrerá a densidade de $\mathfrak{C}_{n}^{H}$.

Sejam

$$
\begin{aligned}
& X=\left\{u \in W^{2, p} \cap W_{0}^{1, p}(\Omega) ;\|u\|_{L^{\infty}(\Omega)}<n+1\right\} \\
& Y=C^{k}(\Omega) \\
& Z=L^{p}(\Omega) .
\end{aligned}
$$

Consideramos a topologia em $X$ induzida pela topologia de $W^{2, p}(\Omega)$. Aplicaremos o Teorema 3.3 para a aplicação $\Phi: X \times Y \longrightarrow Z$ definida do seguinte modo:

$$
\begin{aligned}
\Phi(u, b)(x) & =\Delta u(x)+f(x, u(x))+b(x) \eta(u(x)) \\
& =\Delta u(x)+f(x, u(x))+b(x), \quad x \in \Omega
\end{aligned}
$$


Observe que $X$ e $Y$ são espaços métrico separáveis e $\Phi$ é aplicação de classe $C^{1}$. Além disso, para cada $(u, b) \in X \times Y$,

$$
\begin{gathered}
\frac{\partial \Phi}{\partial u}(u, b): W^{2, p} \cap W_{0}^{1, p}(\Omega) \rightarrow L^{p}(\Omega) \\
\dot{u} \longmapsto \Delta \dot{u}+\frac{\partial f}{\partial u}(x, u(x)) \dot{u}
\end{gathered}
$$

é um operador de Fredholm de índice zero, uma vez que $\frac{\partial f}{\partial u}(\cdot, u(\cdot)) \in C^{0}(\Omega)$.

Note também que $f(x, u)+b(x) \eta(u)$ é uma função de $\mathfrak{C}_{n}^{H}$ se, e somente se, o operador

$$
\dot{u} \rightarrow \Delta \dot{u}+\frac{\partial f}{\partial u}(u, u(x)) \dot{u}: W^{2, p} \cap W_{0}^{1, p} \rightarrow L^{p}(\Omega)
$$

é um isomorfismo para toda função $u \in X$ tal que $\Phi(u, b)=0$, ou seja, se zero é um valor regular de $\Phi(\cdot, b): X \rightarrow Z$.

As hipóteses (1) e (3) do Teorema 3.3 estão nitidamente satisfeitas pela aplicação $\Phi$, restando apenas a verificação de que para cada $(u, b) \in \Phi^{-1}(0)$ a derivada total $D \Phi(u, b)$ é sobrejetora, ou seja, de que vale a hipótese (2)(a).

Sejam $(u, b) \in \Phi^{-1}(0)$ e $h \in L^{p}(\Omega)$. Queremos encontrar $\dot{u} \in W^{2, p} \cap W_{0}^{1, p}(\Omega)$ e $\dot{b} \in C^{k}(\Omega)$ tais que $\frac{\partial \Phi}{\partial u}(u, b) \dot{u}+\frac{\partial \Phi}{\partial b}(u, b) \dot{b}=h$, isto é,

$$
\Delta \dot{u}(x)+\frac{\partial f}{\partial u}(x, u(x)) \dot{u}(x)=h(x)-\dot{b}(x), \quad x \in \Omega .
$$

A equação acima admite solução para $\dot{u}$ desde de que escolhamos $\dot{b}$ satisfazendo

$$
\int_{\Omega}(h(x)-\dot{b}(x)) v_{i}(x) d x=0, \quad i=1, \ldots, m
$$

para uma base $\left\{v_{1}, \ldots, v_{m}\right\}$ de $\mathcal{N}\left(\Delta+\frac{\partial f}{\partial u}(x, u(x))\right)$. Sendo as $v_{i}$ 's linearmente independentes, concluímos sem dificuldades que o sistema

$$
\begin{gathered}
\int_{\Omega} \dot{b}(x) v_{1}(x) d x=\int_{\Omega} h(x) v_{1}(x) d x \\
\vdots \\
\int_{\Omega} \dot{b}(x) v_{m}(x) d x=\int_{\Omega} h(x) v_{m}(x) d x
\end{gathered}
$$

possui uma solução $\dot{b} \in C^{k}(\Omega)$. Logo, $D \Phi(u, b)$ é sobrejetora sempre que $(u, b) \in \Phi^{-1}(0)$ e podemos empregar o Teorema 3.3 para obter um subconjunto denso $A_{f} \subset C^{k}(\Omega)$ com a propriedade de que zero é valor regular de $\Phi(\cdot, b): X \rightarrow Z$ para cada $b \in A_{f}$, ou, em outras palavras, $f(x, u)+b(x) \eta(u) \in \mathfrak{C}_{n}^{H}$ para cada $b \in A_{f}$. 


\subsection{Simplicidade Genérica dos Autovalores Reais}

Vamos considerar o operador diferencial de segunda ordem

$$
A(\lambda)=\sum_{i, j=1}^{n} a_{i j}(x) \frac{\partial^{2}}{\partial x_{i} \partial x_{j}}+\sum_{j=1}^{n} b_{j}(x, \lambda) \frac{\partial}{\partial x_{j}}+c(x, \lambda),
$$

onde $a_{i j}=a_{j i} \in C^{2}$ e $b_{j}(x, \lambda), c(x, \lambda)$ são polinômios em $\lambda$ com coeficientes $C^{1}$ que assumem valores reais quando $\lambda$ é real. Assumiremos que $\sum_{i, j=1}^{n} a_{i j}(x) \xi_{i} \xi_{j} \geq c_{0}|\xi|^{2}$, quaisquer que sejam $x, \xi \in \mathbb{R}^{n}$, para alguma constante $c_{0}>0$.

Dada uma região aberta, limitada, conexa e $C^{3}$-regular $\Omega \subset \mathbb{R}^{n}$, seja $A_{\Omega}(\lambda)$ o operador $A(\lambda)$ definido de $W^{2, p} \cap W_{0}^{1, p}(\Omega)$ em $L^{p}(\Omega)$. Diremos que $\lambda$ é um autovalor de $A_{\Omega}(\lambda)$ se existe uma solução não nula de

$$
A_{\Omega}(\lambda) u=0 \text { em } \Omega, u=0 \text { sobre } \partial \Omega
$$

Diremos que $\lambda$ é um autovalor simples se $\mathcal{N}\left(A_{\Omega}(\lambda)\right)$ é unidimensional e sua imagem, contida em $L^{p}(\Omega)$, não contém $\frac{d}{d \lambda}\left(A_{\Omega}(\lambda)\right) u$ para $u \in \mathcal{N}\left(A_{\Omega}(\lambda)\right) \backslash\{0\}$.

Mostraremos nesta seção que, genericamente no conjunto das regiões $\Omega \subset \mathbb{R}^{n}$ abertas, limitadas, conexas e $C^{3}$-regulares, todos os autovalores de (4.3) são simples. Notemos que nossas hipóteses não excluem o caso em que $A_{\Omega}(\lambda)$ independe de $\lambda$. Neste caso, $\frac{d}{d \lambda}\left(A_{\Omega}(\lambda)\right)=0$ e não podem existir autovalores simples para (4.3). Desse modo, provaremos que $A_{\Omega}(\lambda)$ é genericamente um isomorfismo quando não depende de $\lambda$.

Proposição 4.2. Sejam $\Omega \subset \mathbb{R}^{n}$ uma região aberta, limitada, conexa e $C^{3}$-regular e $h \in$ $\operatorname{Diff}^{3}(\Omega)$. A aplicação

$$
\begin{gathered}
G_{h}:\left(W^{2, p} \cap W_{0}^{1, p}(\Omega) \backslash\{0\}\right) \times \mathbb{R} \longrightarrow L^{p}(\Omega) \\
(u, \lambda) \longmapsto h^{*} A_{h(\Omega)} h^{*-1} u
\end{gathered}
$$

tem zero como valor regular se, e somente se, todos os autovalores reais de

$$
h^{*} A_{h(\Omega)} h^{*-1} u=0 \text { em } \Omega, u=0 \text { sobre } \partial \Omega, u \neq 0
$$

são simples.

Demonstração. Seja $A_{h(\Omega)}^{\prime}(\lambda)=\frac{d}{d \lambda}\left(A_{h(\Omega)}(\lambda)\right)$. A derivada de $G_{h}$ em $(u, \lambda) \in\left(W^{2, p} \cap\right.$ $\left.W_{0}^{1, p}(\Omega) \backslash\{0\}\right) \times \mathbb{R}$ é

$$
\begin{gathered}
D G_{h}(u, \lambda): W^{2, p^{*}} \cap W_{0}^{1, p}(\Omega) \times \mathbb{R} \longrightarrow L^{p}(\Omega) \\
(\dot{u}, \dot{\lambda}) \longmapsto h^{*} A_{h(\Omega)} h^{*-1} \dot{u}+\dot{\lambda} h^{*} A_{h(\Omega)}^{\prime} h^{*-1} u
\end{gathered}
$$

Pelo Exemplo 4 do Capítulo 2, $A_{h(\Omega)}(\lambda): W^{2, p} \cap W_{0}^{1, p}(h(\Omega)) \rightarrow L^{p}(h(\Omega))$ é um operador de Fredholm com índice 0 . Assim, o operador linear $(\dot{u}, \dot{\lambda}) \in W^{2, p} \cap W_{0}^{1, p}(\Omega) \times \mathbb{R} \longmapsto$ 
$h^{*} A_{h(\Omega)}(\lambda) h^{*-1} \dot{u} \in L^{p}(\Omega)$ é um operador de Fredholm com índice 1 . Observando agora que o operador $(\dot{u}, \dot{\lambda}) \in W^{2, p} \cap W_{0}^{1, p}(\Omega) \times \mathbb{R} \longmapsto \dot{\lambda} h^{*} A_{h(\Omega)} h^{*-1} u \in L^{p}(\Omega)$ é compacto, concluímos, pelo Teorema 2.3, que $D G_{h}(u, \lambda)$ é Fredholm com índice 1 .

Suponha que zero é valor regular de $G_{h}$. Sejam $\lambda$ um autovalor de (4.4) e $u \in W^{2, p} \cap$ $W_{0}^{1, p}(\Omega) \backslash\{0\}$ tal que $h^{*} A_{h(\Omega)} h^{*-1} u=0$. Como $\operatorname{ind}\left(D G_{h}(u, \lambda)\right)=1$ e $D G_{h}(u, \lambda)$ é sobrejetora, chegamos à conclusão de que $\mathcal{N}\left(D G_{h}(u, \lambda)\right)=[(u, 0)]$, o que fornece que $\operatorname{dim} \mathcal{N}\left(h^{*} A_{h(\Omega)} h^{*-1}\right)=$ 1 e que $h^{*} A_{h(\Omega)}^{\prime} h^{*-1} u \notin \mathcal{R}\left(h^{*} A_{h(\Omega)} h^{*-1}\right)$.

Reciprocamente, suponha que todos os autovalores de (4.4) são simples. Tome $(u, \lambda) \in$ $G_{h}^{-1}(0)$. Então $\mathcal{N}\left(h^{*} A_{h(\Omega)}(\lambda) h^{*-1}=[u]\right.$ e $\dot{\lambda} h^{*} A_{h(\Omega)}^{\prime}(\lambda) h^{*-1} u \notin \mathcal{R}\left(h^{*} A_{h(\Omega)}(\lambda) h^{*-1} \dot{u}\right.$ quaisquer que sejam $\dot{\lambda} \neq 0$ e $\dot{u} \in W^{2, p} \cap W_{0}^{1, p}(\Omega)$, o que mostra que mathcal $N\left(D G_{h}(u, \lambda)\right)=[(u, 0)]$. Por fim, recorrendo ao fato de que $\operatorname{ind}\left(D G_{h}(u, \lambda)\right)=1$, obtemos que $D G_{h}(u, \lambda)$ é sobrejetora.

Proposição 4.3. $\lambda$ é um autovalor simples de (4.4) se, e somente se, $\lambda$ é um autovalor simples de

$$
A_{h(\Omega)}(\lambda) v=0 \text { em } h(\Omega), v=0 \text { sobre } \partial h(\Omega), v \neq 0 .
$$

Demonstração. Observemos que

$$
h^{*} A_{h(\Omega)}(\lambda) h^{*-1} u=0 \Longleftrightarrow A_{h(\Omega)}(\lambda)\left(h^{*-1} u\right)=0
$$

já que $h^{*}$ é um isomorfismo. Então $v=h^{*-1} u \in W^{2, p} \cap W_{0}^{1, p}(\Omega)(h(\Omega))$ é autofunção de (4.5) associada a $\lambda$ se, e somente se, $u \in W^{2, p} \cap W_{0}^{1, p}(\Omega)$ é autofunção de (4.4) associada a $\lambda$, de maneira que $\mathcal{N}\left(h^{*} A_{h(\Omega)}(\lambda) h^{*-1}\right) \subset W^{2, p} \cap W_{0}^{1, p}(\Omega)$ é unidimensional se, e somente se, $\mathcal{N}\left(A_{h(\Omega)}(\lambda)\right) \subset W^{2, p} \cap W_{0}^{1, p}(h(\Omega))$ é unidimensional.

Para finalizarmos a demonstração, basta verificarmos que

$$
\frac{d}{d \lambda}\left(A_{h(\Omega)}(\lambda)\right) v \in \mathcal{R}\left(A_{h(\Omega)}(\lambda)\right) \Longleftrightarrow \frac{d}{d \lambda}\left(h^{*} A_{h(\Omega)}(\lambda) h^{*-1}\right) u \in \mathcal{R}\left(h^{*} A_{h(\Omega)}(\lambda) h^{*-1}\right)
$$

Como. $\frac{d}{d \lambda}\left(h^{*} A_{h(\Omega)}(\lambda) h^{*-1}\right)=h^{*} \frac{d}{d \lambda}\left(A_{h(\Omega)}(\lambda)\right) h^{*-1}$ e $h^{*}$ é um isomorfismo, temos que

$$
\begin{aligned}
\frac{d}{d \lambda}\left(A_{h(\Omega)}(\lambda)\right) v \in \mathcal{R}\left(A_{h(\Omega)}(\lambda)\right) & \Longleftrightarrow \frac{d}{d \lambda}\left(A_{h(\Omega)}(\lambda)\right) h^{*-1} u \in \mathcal{R}\left(A_{h(\Omega)}\right) h^{*-1} u \in \mathcal{R}\left(A_{h(\Omega)}(\lambda)\right) \\
& \Longleftrightarrow h^{*} \frac{d}{d \lambda}\left(A_{h(\Omega)}(\lambda)\right) h^{*-1} u \in \mathcal{R}\left(h^{*} A_{h(\Omega)}(\lambda) h^{*-1}\right)
\end{aligned}
$$

do que segue o resultado desejado.

Os resultados enunciados na Proposição 4.2 e na Proposição 4.3 evidenciam que para obtermos a simplicidade genérica dos autovalores de (4.3) é suficiente provarmos que, fixada uma região aberta, limitada, conexa e $C^{3}$-regular $\Omega \subset \mathbb{R}^{n}$, para a "maioria" dos difeomorfismos 
$h \in \operatorname{Diff}^{3}(\Omega)$ a aplicação $G_{h}$ tem o zero como valor regular. Para prová-lo, vamos empregar o Teorema 3.3 para a aplicação diferenciável

$$
\begin{gathered}
F:\left(W^{2, p} \cap W_{0}^{1, p}(\Omega) \backslash\{0\}\right) \times \mathbb{R} \times \operatorname{Diff}^{3}(\Omega) \longrightarrow L^{p}(\Omega) \\
(u, \lambda, h) \longmapsto h^{*} A_{h(\Omega)}(\lambda) h^{*-1} u .
\end{gathered}
$$

Notando que para $h$ fixado a aplicação $(u, \lambda) \longmapsto F(u, \lambda, h)$ é um operador de Fredholm de índice 1 e atentando para o fato de que todos os espaços envolvidos são șeparáveis, concluímos que $F$ cumpre as hipóteses (1) e (3) do Teorema 3.3. Resta-nos apenas a verificação de que zero é valor regular de $F$, o que faremos por absurdo.

Suponha que existe um ponto crítico $(u, \lambda, h)$ de $F$ tal que $F(u, \lambda, h)=0$. Seja $\Omega_{l}=h(\Omega)$ e defina uma nova aplicação diferenciável

$$
\begin{gathered}
\tilde{F}:\left(W^{2, p} \cap W_{0}^{1, p}\left(\Omega_{1}\right) \backslash\{0\}\right) \times \mathbb{R} \times \operatorname{Diff}^{3}\left(\Omega_{1}\right) \longrightarrow L^{p}\left(\Omega_{1}\right) \\
(v, \lambda, g) \longmapsto g^{*} A_{h\left(\Omega_{1}\right)}(\lambda) g^{*-1} .
\end{gathered}
$$

Usando que $(g \circ h)^{*}=h^{*} g^{*}$ obtemos que

$$
h^{*-1} F\left(h^{*} v, \lambda, g \circ h\right)=h^{*-1}\left(h^{*} g^{*}\right) A_{g\left(\Omega_{1}\right)}(\lambda)\left(g^{*-1} h^{*-1}\right) h^{*-1} v=\tilde{F}(v, \lambda, g) .
$$

Assim, levando em consideração que $h^{*}$ é um isomorfismo, concluímos que $\left(h^{*-1} u, \lambda, i \Omega_{1}\right)$ é um ponto crítico de $\tilde{F}, \operatorname{com} \tilde{F}\left(h^{*-1} u, \lambda, i_{\Omega_{1}}\right)=0$. Procede, portanto, a seguinte

Observação: Não há perda de generalidade em supor que o ponto crítico $(u, \lambda, h)$ de $F$ tal que $F(u, \lambda, h)=0$ tem $h=i_{\Omega}$, o que simplifica nossos cálculos e notações.

Calculando então a derivada de $F$ no ponto crítico $\left(u, \lambda, i_{\Omega}\right)$ com o auxílio do Teorema 3.2 , obtemos que o operador

$$
\begin{aligned}
D F\left(u, \lambda, i_{\Omega}\right): W^{2, p} \cap W_{0}^{1, p}(\Omega) \times \mathbb{R} \times C^{3}\left(\Omega, \mathbb{R}^{n}\right) & \longrightarrow L^{p}(\Omega) \\
(\dot{u}, \dot{\lambda}, \dot{h}) & \longmapsto A_{\Omega}(\lambda)(\dot{u}-\dot{h} \cdot \nabla u)+\dot{\lambda} A_{\Omega}^{\prime}(\lambda)(u)
\end{aligned}
$$

não é sobrejetor.

A imagem do subespaço $\left\{(\dot{u}, \dot{\lambda}, \dot{h}) ; \dot{u} \in W^{2, p} \cap W_{0}^{1, p}(\Omega), \dot{\lambda}=0, \dot{h}=0\right\}$ pela aplicação $D F\left(u, \lambda, i_{\Omega}\right)$, coincide com a imagem do operador de Fredholm $A_{\Omega}(\lambda)$ (que possui codimensão finita) o que nos permite concluir que $\mathcal{R}\left(D F\left(u, \lambda, i_{\Omega}\right)\right)$ tem codimensão finita e, conseqüentemente, é fechada. Logo, existe $\psi \in L^{q}(\Omega) \backslash\{0\}$, onde $1<p<\infty$ é tal que $p^{-1}+q^{-1}=1$, satisfazendo

$$
\int_{\Omega} \psi D F\left(u, \lambda, i_{\Omega}\right)(\dot{u}, \dot{\lambda}, \dot{h})=0
$$

quaisquer que sejam $\dot{u} \in W^{2, p} \cap W^{1, p}(\Omega), \dot{\lambda} \in \mathbb{R}$ e $\dot{h} \in C^{3}\left(\Omega, \mathbb{R}^{n}\right)$. 
Em particular, fixando $\dot{\lambda}=0$ e $\dot{h}=0$, temos

$$
\int_{\Omega} \psi A_{\Omega}(\lambda) \dot{u}=0
$$

para toda função $\dot{u} \in W^{2, p} \cap W_{0}^{1, p}(\Omega)$, do que decorre, se aplicamos o Teorema 1.3, que $\psi \in$ $W^{2, p} \cap W^{1, p}(\Omega)$ e é solução forte de

$$
A_{\Omega}^{*}(\lambda) \psi=0 \text { em } \Omega, \psi=0 \text { sobre } \partial \Omega .
$$

Mais ainda, pelo Teorema $1.5, u, \psi \in W^{3, p}(\Omega)$, o que fornece, se apelamos ao Teorema 1.6, que $\psi, u \in C^{2}(\Omega)$.

Então, para cada $h \in C^{3}\left(\Omega, \mathbb{R}^{n}\right)$ temos

$$
\begin{aligned}
0 & =\int_{\Omega} \psi A_{\Omega}(\lambda) \dot{h} \cdot \nabla u \\
& =\int_{\Omega}\left\{\psi A_{\Omega}(\lambda) \dot{h} \cdot \nabla u-A_{\Omega}^{*}(\lambda) \psi \dot{h} \cdot \nabla u\right\} \\
& =-\int_{\partial \Omega} \dot{h} \cdot \nabla u \sum_{i, j=1}^{n} \psi_{x_{i}} a_{i j}(x) N_{j} \\
& =-\int_{\partial \Omega} \frac{\partial u}{\partial N} \dot{h} \cdot N \sum_{j=1}^{n} N_{j} \nabla \psi \cdot\left(a_{1 j}(x), \ldots, a_{n j}(x)\right) \\
& =-\int_{\partial \Omega} \frac{\partial \psi}{\partial N} \frac{\partial u}{\partial N} \dot{h} \cdot N\left(\sum_{i, j=1}^{n} a_{i j}(x) N_{i} N_{j}\right),
\end{aligned}
$$

do que vemos que $\frac{\partial u}{\partial N} \frac{\partial \psi}{\partial N}=0$ sobre $\partial \Omega$, uma vez que $\sum_{i, j=1}^{n} a_{i j}(x) N_{i} N_{j} \geq c_{0}|N|^{2} \geq c_{0}$.

Admita que para algum $x_{0} \in \partial \Omega$ tenhamos $\frac{\partial u}{\partial N}\left(x_{0}\right) \neq 0$. Então $\frac{\partial u}{\partial N} \neq 0$ em uma vizinhança $V$ de $x_{0}$ em $\partial \Omega$, implicando que $\frac{\partial \psi}{\partial N}=0$ nesta vizinhança. Assim, temos $\psi=0$ e $\frac{\partial \psi}{\partial N}=0 \mathrm{em} V$, $\operatorname{com} A_{\Omega}^{*}(\lambda) \psi=0 \mathrm{em} \Omega$, do que resulta, pelo Teorema 1.2 , que $\psi=0 \mathrm{em} \Omega$, contrariando nossa cscolha de $\psi$. Logo, $\frac{\partial u}{\partial N}=0$ em $\partial \Omega$. Aplicando agora o Teorema 1.2 para $u$, somos levados à conclusão de que $u=0$ em $\Omega$, uma contradição.

Portanto zero é valor regular da aplicação $F$ e o Teorema 3.3 garante, como queríamos, a simplicidade genérica dos autovalores reais.

\subsection{Simplicidade Genérica das Soluções de $\Delta u+f(x, u, \nabla u)=0$}

Nesta seção, fixada uma função $f: \mathbb{R}^{n} \times \mathbb{R} \times \mathbb{R}^{n} \rightarrow \mathbb{R}(n \geq 2)$ de classe $C^{2}$, perseguiremos o objetivo de provar que, genericamente no conjunto das regiöes $\Omega \subset \mathbb{R}^{n}$ abertas, limitadas, conexas e $C^{3}$-regulares, todas as soluções $u$ de

$$
\begin{aligned}
\Delta u+f(x, u, \nabla u) & =0, x \in \Omega \\
u & =0, x \in \partial \Omega
\end{aligned}
$$


são simples, ou seja, a linearização

$$
L_{u}: \dot{u} \longmapsto \Delta \dot{u}+\frac{\partial f}{\partial p}(\cdot, u, \nabla u) \cdot \nabla \dot{u}+\frac{\partial f}{\partial u}(\cdot, u, \nabla u) \dot{u}
$$

é um isomorfismo.

Para isto, vamos considerar $X$ o conjunto de todas as regiōes $C^{3}$-difeomorfas a uma região $\Omega$, conexa, limitada e de classe $C^{3}$ fixada, isto é,

$$
X=\left\{h(\Omega) ; h \in \operatorname{Diff}^{3}(\Omega)\right\} .
$$

Introduzimos uma topologia em $X$ definindo uma base de vizinhanças para uma região $\Omega^{\prime}$ em $X$ por

$$
\left\{h\left(\Omega^{\prime}\right) ;\left\|h-i_{\Omega^{\prime}}\right\|_{C^{3}\left(\Omega, \mathbb{R}^{n}\right)}<\epsilon\right\}
$$

para $\epsilon>0$ suficientemente pequeno. O espaço topológico obtido é um espaço métrico completo, fato provado por Michelleti em [15].

$\mathrm{Na}$ verdade, provaremos que todas as soluções de (4.6), (4.7) em $h(\Omega)$ são simples para $h$ pertencente ao complementar de um subconjunto magro $\mathfrak{M} \subset \operatorname{Diff}^{3}(\Omega)$. Como o conjunto $\mathfrak{M}$ de mergulhos excluídos será definido pelas propriedades das imagens dos mergulhos, $\mathfrak{M}$ será invariante por composição com difeomorfismos de classe $C^{3}$ de $\Omega$ em $\Omega$, o que assegura que o subconjunto $\{h(\Omega) ; h \in \mathfrak{M}\}$ também é magro em $X$.

É consequência imediata do teorema da função inversa em espaços de Banach que se todas as soluções de (4.6), (4.4) em uma dada região $\Omega$ são simples, tais soluções formam um conjunto discreto. Em nosso caso, a limitação de $f$ garante ainda mais: o conjunto das soluções de (4.6), (4.4), se $\Omega$ for uma região em que todas as soluções são simples, é finito. Realmente, se $\Delta u+f(x, u, \nabla u)=0$, temos que

$$
\|\Delta u\|_{L^{p}(\Omega)}=\|f(\cdot, u, \nabla u)\|_{L^{p}(\Omega)} \leq M,
$$

onde $M=m(\Omega) \sup \left\{|f(x, y, z)| ;(x, y, z) \in \mathbb{R}^{n} \times \mathbb{R} \times \mathbb{R}^{n}\right\}$. Sendo $\Delta: W^{2, p} \cap W_{0}^{1, p}(\Omega) \longrightarrow L^{p}(\Omega)$ um isomorfismo, existe $m>0$ satisfazendo

$$
M \geq\|\Delta u\|_{L^{p}(\Omega)} \geq m\|u\|_{W^{2, p} \cap W_{0}^{1, p}(\Omega)},
$$

do que decorre que

$$
\|u\|_{W^{2, p} \cap W_{0}^{1, p}(\Omega)} \leq \frac{M}{m} .
$$

Como a imersão de $W^{2, p} \cap W_{0}^{1, p}(\Omega)$ em $L^{p}(\Omega)$ é compacta e o conjunto das soluçōes é discreto, o número de soluções é necessariamente finito.

Proposiçāo 4.4. Fixada $h \in \operatorname{Diff}^{3}(\Omega)$, considere $F_{h}: W^{2, p} \cap W_{0}^{1, p}(\Omega) \rightarrow L^{p}(\Omega)$ a aplicação dada por $F_{h} u=h^{*}(\Delta+f) h^{*-1} u$. Uma função $u \in W^{2, p} \cap W_{0}^{1, p}(\Omega)$ é solução simples de $F_{h} v=0$ se, e somente se, $h^{*-1} u$ é uma solução simples de (4.6), (4.7) em $h(\Omega)$. 
Demonstração. O fato de $h^{*}$ ser isomorfismo assegura que se $u \in W^{2, p} \cap W_{0}^{1, p}(\Omega)$ então $F_{h} u=0$ se, somente se, $(\Delta+f)\left(h^{*-1} u\right)=0$ (ou seja, se $h^{*-1} u$ é solução de (4.6), (4.7) em $h(\Omega)$ ). Além disso, a linearização $h^{*} L_{h^{*-1} u} h^{*-1}$ de $F_{h}$ no ponto $u$ é um isomorfismo de $W^{2, p} \cap W_{0}^{1, p}(\Omega)$ em $L^{p}(\Omega)$ se, e somente se, $L_{h^{*-1} u}$ é um isomorfismo de $W^{2, p} \cap W_{0}^{1, p}(h(\Omega))$ em $L^{p}(h(\Omega))$, conclusão a que chegamos levando em consideração que $L_{h^{*} u}$ é Fredholm com índice zero.

Proposição 4.5. Sejam $h \in \operatorname{Diff}^{3}(\Omega)$ fixada e $F_{h}$ como na Proposição 4.4. Se 0 é um valor regular de $F_{h}$ entäo todas as soluçồes de (4.6),(4.7) em $h(\Omega)$ são simples.

Demonstração. Suponha 0 um valor regular de $F_{h}$ e seja $v \in W^{2, p} \cap W_{0}^{1, p}(h(\Omega))$ uma solução de (4.6),(4.7). Então $u=h^{*} v$ satisfaz $F_{h} u=0$. Logo, $h^{*} L_{v} h^{*-1}$ é sobrejetora, do que resulta imediatamente que $L_{v}$ é também sobrejetora. Agora, $L_{v}$ é Fredholm com índice zero, o que nos permite concluir que $L_{v}$ é um isomorfismo.

As proposições acima mostram essencialmente que o estudo da simplicidade genérica se reduz ao problema de provarmos que para a "maioria" dos difeomorfismos $h \in \operatorname{Diff}^{3}(\Omega)$, a aplicação $F_{h}$ tem o zero como valor regular. Para solucioná-lo, considere $E_{1}$ e $E_{2}$ os seguintes subconjuntos de $C^{3}\left(\Omega, \mathbb{R}^{n}\right)$ :

$$
\begin{aligned}
& E_{1}=\left\{h \in C^{3}\left(\Omega, \mathbb{R}^{n}\right) ; f(x, 0,0) \equiv 0 \text { em uma vizinhança de } \partial h(\Omega)\right\} \\
& E_{2}=\left\{h \in C^{3}\left(\Omega, \mathbb{R}^{n}\right) ; f(x, 0,0) \neq 0 \text { para algum } x \in \partial h(\Omega)\right\}
\end{aligned}
$$

Se $h_{0} \in E_{1}$, seja $W_{h_{0}}$ uma vizinhança de $\partial h_{0}(\Omega)$ em que $f(x, 0,0) \equiv 0$. É possível escolher uma vizinhança $V_{h_{0}}$ de $h_{0}$ em $C^{3}\left(\Omega, \mathbb{R}^{n}\right)$ tal que se $h \in V_{h_{0}}$ então $\partial h(\Omega) \subset W_{h_{0}}$. Desse modo, $V_{h_{0}} \subset E_{1}$. Portanto, $E_{1}$ é aberto.

Se $h_{0} \in E_{2}$, existe $x \in \partial h_{0}(\Omega) \operatorname{com} f(x, 0,0) \neq 0$. Seja $U$ uma vizinhança de $x$ em $\mathbb{R}^{n}$ com a propriedade de que $f(x, 0,0) \neq 0$ para $x \in U$. Neste caso, podemos selecionar uma vizinhança $V_{h_{0}}$ de $h_{0}$ em $C^{3}\left(\Omega, \mathbb{R}^{n}\right)$ tal que se $h \in V_{h_{0}}$ então $\partial h(\Omega) \cap U \neq \varnothing$, o que significa que $V_{h_{0}} \subset E_{2}$. Logo, $E_{2}$ é aberto.

Tomando $E=E_{1} \cup E_{2}$, obtemos um subconjunto aberto e denso de $C^{3}\left(\Omega, \mathbb{R}^{n}\right)$. Sendo assim, $D=E \cap \operatorname{Diff}^{3}(\Omega)$, que pode ser escrito como a reunião disjunta $D=D_{1} \cup D_{2}$, em que $D_{1}=E_{1} \cap \operatorname{Diff}^{3}(\Omega)$ e $D_{2}=E_{2} \cap \operatorname{Diff}^{3}(\Omega)$, é aberto c denso em $\operatorname{Diff}^{3}(\Omega)$.

Para $h \in D_{1}$, seja $W_{h}$ uma vizinhança tubular de $\partial h(\Omega)$ em que tenhamos $f(x, 0,0) \equiv 0$. Podemos escolher uma vizinhança $V_{h}$ de $h$ em $C^{3}\left(\Omega, \mathbb{R}^{n}\right)$, contida em $D_{1}$, de maneira que se $\tilde{h} \in V_{h}$ então $\partial \tilde{h}(\Omega)$ está contida em $W_{h}$. Se $h \in D_{2}$, seja $V_{h}$ uma vizinhança de $h$ em $C^{3}\left(\Omega, \mathbb{R}^{n}\right)$ contida em $D_{2}$. O conjunto de tais vizinhanças constitui uma cobertura aberta 
para $D$ que, por ser $D$ um espaço de Lindelöf, admite uma subcobertura enumerável, digamos $\left\{V_{h_{1}}, V_{h_{2}}, \ldots, V_{h_{k}}, \ldots\right\}$.

A partir de agora, nosso propósito será provar que para cada $k \in \mathbb{N}$ existe um subconjunto magro $H_{k}$ de $D$, contido em $V_{h_{k}}$, tal que se $h \in V_{h_{k}} \backslash H_{k}$ então todas as soluções de $(4.6),(4.7)$ em $h(\Omega)$ são simples. Se o fizermos, teremos provado a simplicidade genérica das soluções de (4.6), (4.7), uma vez que $\operatorname{Diff}^{3}(\Omega) \backslash\left(\cup_{k=1}^{\infty}\left(V_{h_{k}} \backslash H_{k}\right)\right)=D^{c} \cup\left(\cup_{k=1}^{\infty} H_{k}\right)$ é magro em $\operatorname{Diff}^{3}(\Omega)$.

Fixe $k \in \mathbb{N}$ arbitrário e considere a aplicação

$$
\begin{aligned}
& F: W^{2, p} \cap W_{0}^{1, p}(\Omega) \times \operatorname{Diff}^{3}(\Omega) \rightarrow L^{p}(\Omega) \\
& (u, h) \longmapsto h^{*}(\Delta+f) h^{*-1} u
\end{aligned}
$$

para algum $n<p<\infty$, de modo a termos $W^{2, p}(\Omega) \subset C^{1}(\Omega)$. Aplicaremos o Teorema 3.3 para concluir que existe um subconjunto magro $H_{k}$ em $V_{h_{k}}$ (e, portanto, magro em $D$ ) tal que se $h \in V_{h_{k}} \backslash H_{k}$ então zero é valor regular de $F(\cdot, h): W^{2, p} \cap W_{0}^{1, p}(\Omega) \rightarrow L^{p}(\Omega)$. Recordando - Exemplo 4 do Capítulo 2 e levando em consideração que $W^{2, p} \cap W_{0}^{1, p}(\Omega)$ e $V_{h_{k}}$ são espaços separáveis, vemos facilmente que a aplicação $F$ satisfaz as hipóteses (1) e (3) do Teorema 3.3. Quanto à hipótese (2), será preciso proceder com maior cuidado.

Assuma que exista $\left(u, i_{\Omega}\right)$ ponto crítico de $F \operatorname{com} F\left(u, i_{\Omega}\right)=0$. Então a diferencial de $F$ no ponto $\left(u, i_{\Omega}\right)$,

$$
\begin{aligned}
& D F\left(u, i_{\Omega}\right): W^{2, p} \cap W_{0}^{1, p}(\Omega) \times C^{3}\left(\Omega, \mathbb{R}^{n}\right) \rightarrow L^{p}(\Omega) \\
& (\dot{u}, \dot{h}) \mapsto L_{u}(\dot{u}-\dot{h} \cdot \nabla u),
\end{aligned}
$$

não é sobrejetora. Observe que $\mathcal{R}\left(D F\left(u, i_{\Omega}\right)\right)=\mathcal{R}\left(L_{u}\right)$ é subespaço fechado de $L^{p}(\Omega)$ pois $L_{u}$ é Fredholm. Portanto, se $p^{-1}+q^{-1}=1$, existe uma função não nula $\psi \in L^{q}(\Omega)$ para a qual

$$
\int_{\Omega} \psi L_{u}(\dot{u}-\dot{h} \cdot \nabla u)=0
$$

sejam quais forem $\dot{u} \in W^{2, p} \cap W^{1, p}(\Omega)$ e $\dot{h} \in C^{3}\left(\Omega, \mathbb{R}^{n}\right)$.

Como $u$ é solução de (4.6), (4.7) e $f \in C^{2}$, pela observaçāo que fizemos no Capítulo 1 , temos que $u \in C^{2, \alpha}$ para todo $\alpha<1$, resultando que os coeficientes de $L_{u}$ são, no mínimo, de classe $C^{2}$.

Fixando $\dot{h}=0$, obtemos que para cada $\dot{u} \in W^{2, p} \cap W_{0}^{1, p}(\Omega)$

$$
\int_{\Omega} \psi L_{u} \dot{u}=0
$$

do que decorre que $\psi \in W^{2, p} \cap W_{0}^{1, p}(\Omega)$ e $L^{*} \psi=0$, segundo o que nos permite concluir o Teorema 1.3. Observe que, como $u, \psi$ é de classe $C^{2, \alpha}$. 
Tomando $\dot{u}=0$, verificamos que

$$
\begin{aligned}
0 & =\int_{\Omega} \psi L_{u}(\dot{h} \cdot \nabla u) \\
& =\int_{\Omega}\left\{\psi L_{u}(\dot{h} \cdot \nabla u)-(\dot{h} \cdot \nabla u) L_{u}^{*} \psi\right\} \\
& =\int_{\partial \Omega}\left\{\psi \frac{\partial(\dot{h} \cdot \nabla u)}{\partial N}-\frac{\partial \psi}{\partial N}(\dot{h} \cdot \nabla u)+\psi(\dot{h} \cdot \nabla u) \frac{\partial f}{\partial p} \cdot N\right\} \\
& =-\int_{\partial \Omega} \dot{h} \cdot N \frac{\partial \psi}{\partial N} \frac{\partial u}{\partial N},
\end{aligned}
$$

o que nos leva à conclusão de que $\frac{\partial \psi}{\partial N} \frac{\partial u}{\partial N} \equiv 0$ em $\partial \Omega$.

Agora, $L_{u}^{*} \psi=0$ significa precisamente que

$$
\Delta \psi-\frac{\partial f}{\partial p}(x, u, \nabla u) \cdot \nabla \psi+\left(\frac{\partial f}{\partial u}(x, u, \nabla u)-\operatorname{div}\left(\frac{\partial f}{\partial p}(x, u, \nabla u)\right)\right) \psi=0,
$$

o que implica

$$
\begin{aligned}
|\Delta \psi(x)| & \leq\left|\frac{\partial f}{\partial p}(x, u(x), \nabla u(x)) \cdot \nabla \psi(x)\right|+\left|\frac{\partial f}{\partial u}(x, u(x), \nabla u(x)) \psi(x)\right| \\
& +\left|\sum_{i=1}^{n} \frac{\partial^{2} f}{\partial x_{i} \partial p_{i}}(x, u(x), \nabla u(x)) \psi(x)\right|, \quad x \in \Omega .
\end{aligned}
$$

A limitação de $\Omega$ e o fato de que $f \in C^{2}(\Omega \times \mathbb{R})$ e $u \in C^{2}(\Omega)$ garantem que

$$
|\Delta \psi(x)| \leq C(|\nabla \psi(x)|+|\psi(x)|), \quad x \in \Omega
$$

onde

$$
C=\sup _{x \in \bar{\Omega}}\left\{\left|\frac{\partial f}{\partial p}(x, u(x), \nabla u(x))\right|,\left|\frac{\partial f}{\partial u}(x, u(x), \nabla u(x))\right|,\left|\sum_{i=1}^{n} \frac{\partial^{2} f}{\partial x_{i} \partial p_{i}}(x, u(x), \nabla u(x))\right|\right\} .
$$

Como $\psi \in W^{2, p} \cap W_{0}^{1, p}(\Omega) \backslash\{0\}$ satisfaz a desigualdade acima, temos pelo Teorema 1.2 que $\frac{\partial \psi}{\partial N} \neq 0$ quase sempre em $\partial \Omega$ e, conseqüentemente, $\frac{\partial u}{\partial N}=0$ quase sempre em $\partial \Omega$.

Recordemos que há duas possibilidades para $V_{h_{k}}$ : ou $V_{h_{k}} \subset D_{1}$ ou $V_{h_{k}} \subset D_{2}$. Se $V_{h_{k}} \subset D_{1}$, existe uma vizinhança $W_{h_{k}}$ de $\partial \Omega$ tal que $f(x, 0,0)=0$ para todo $x \in W_{h_{k}}$. Desse modo, para alguma constante $K>0$,

$$
\begin{aligned}
|\Delta u(x)| & =\mid f(x, u(x), \nabla u(x) \mid \\
& =|f(x, u(x), \nabla u(x))-f(x, 0,0)| \\
& \leq K(|u(x)|+|\nabla u(x)|)
\end{aligned}
$$

sempre que $x \in \Omega \cap W_{h_{k}}$.

Considerando que $u=\frac{\partial u}{\partial N}=0$ quase sempre em $\partial \Omega$, podemos recorrer ao Teorema 1.2 para obtermos que $u=0$ em $\Omega \cap W_{h_{k}}$. 
Defina $u_{0}: \Omega \cup V \rightarrow \mathbb{R}$ por

$$
u_{0}= \begin{cases}u(x) & , x \in \Omega \backslash W_{h_{k}} \\ 0 & , x \in\left(\Omega \cap W_{h_{k}}\right) \cup\left(W_{h_{k}} \backslash \Omega\right)\end{cases}
$$

Observe que para cada $h \in V_{h_{k}},\left.u_{0}\right|_{h(\Omega)}$ é solução de (4.6), (4.7) em $h(\Omega)$. Além disso, se $u_{1}$ é outra solução de $(4.6),(4.7)$ em $h(\Omega), \operatorname{com} \frac{\partial u_{1}}{\partial N}=0$ sobre $\partial h(\Omega)$, então $v=u_{0}-u_{1}$ satisfaz

$$
\begin{gathered}
\Delta v+f\left(x, u_{0}, \nabla u_{0}\right)-f\left(x, u_{1}, \nabla u_{1}\right)=0 \text { em } h(\Omega), \\
v=0 \text { e } \frac{\partial v}{\partial N}=0 \text { sobre } \partial h(\Omega) .
\end{gathered}
$$

Ainda, para algum $M>0$ temos

$$
\begin{aligned}
|\Delta v(x)| & =\mid f\left(x, u_{0}(x), \nabla u_{0}(x)-f\left(x, u_{1}(x), \nabla u_{1}(x) \mid\right.\right. \\
& \leq M(|v(x)|+|\nabla v(x)|)
\end{aligned}
$$

qualquer que seja $x \in h(\Omega)$. Segue do Teorema 1.2 que $v=0$ em $h(\Omega)$, ou seja, $u_{0}=u_{1}$.

Seja então $\tilde{F}: W^{2, p} \cap W_{0}^{1, p}(\Omega) \backslash\left\{u_{0}\right\} \times V_{h_{k}} \longrightarrow L^{p}(\Omega)$ a restrição de $F$ ao conjunto $W^{2, p} \cap$ $W_{0}^{1, p}(\Omega) \backslash\left\{u_{0}\right\} \times V_{h_{k}}$. Pela discussão acima, 0 é valor regular de $\tilde{F}$. Aplicando o Teorema 3.3 à aplicação $\tilde{F}$, obtemos um subconjunto magro $\tilde{H}_{k} \subset V_{h_{k}}$ tal que se $h \in V_{h_{k}} \backslash \tilde{H}_{k}$, todas as soluçōes de (4.6), (4.7) em $h(\Omega)$ são simples, exceto possivelmente a restrição de $u_{0}$ a $h(\Omega)$.

Contudo, sabemos pelo exemplo apresentado na Seção 4.2 que existe um subconjunto magro $H \subset V_{h_{k}}$ tal que $L_{h^{*-1} u_{0}}$ é um isomorfismo para toda $h \in V_{h_{k}} \backslash H$. Por fim, escolhendo $H_{k}=$ $\bar{H}_{k} \cup H$, conseguimos um subconjunto magro de $V_{h_{k}}$ com a propriedade de que todas as soluçōes de (4.6), (4.7) em $h(\Omega)$ sãọ simples para $h \in V_{h_{k}} \backslash H_{k}$.

Admita agora que $V_{h_{k}} \subset D_{2}$. Neste caso, $f(x, 0,0) \neq 0$ para algum $x \in \partial \Omega$. Mostraremos que existe um subconjunto $O \subset V_{h_{k}}$, fechado e com interior vazio, tal que se $\tilde{F}: W^{2, p} \cap W_{0}^{1, p}(\Omega) \times$ $\left(V_{h_{k}} \backslash O\right) \longrightarrow L^{p}(\Omega)$ é a restrição de $F$ ao conjunto $W^{2, p} \cap W_{0}^{1, p}(\Omega) \times\left(V_{h_{k}} \backslash O\right)$ então zero é valor regular de $\tilde{F}$.

Considere $G$ a seguinte aplicação de classe $C^{1}$ :

$$
\begin{aligned}
& G: W_{0}^{2, p}(\Omega) \times V_{h_{k}} \longrightarrow L^{p}(\Omega) \\
& (u, h) \longmapsto h^{*}(\Delta+f) h^{*-1} u .
\end{aligned}
$$

Observe que uma função $u \in W^{2, p} \cap W_{0}^{1, p}(\Omega)$ pertence a $W_{0}^{2, p}(\Omega)$ se, e somente se, $\frac{\partial u}{\partial N}=0$ em $\partial \Omega$. Com isso, teremos alcançado nosso objetivo se provarmos que para todo difeomorfismo $h \in V_{h_{k}}$, exceto aqueles em um subconjunto fechado e com interior vazio $O$, temos $G(u, h) \neq 0$ qualquer que seja $u \in W_{0}^{2, p}(\Omega)$. Obteremos tal prova aplicando a $G$ o Corolário 3.7. 
Suponha que $G\left(u, i_{\Omega}\right)=0$. Afirmamos que $\frac{\partial G}{\partial u}\left(u, i_{\Omega}\right)=\left.L_{u}\right|_{W_{0}^{2, p}(\Omega)}$ tem núcleo trivial. Com efeito, seja $v \in W_{0}^{2, p}(\Omega)$ tal que $L_{u} v=0$. Então

$$
\begin{aligned}
|\Delta v(x)| & =\left|\frac{\partial f}{\partial p}(x, u(x), \nabla u(x)) \cdot \nabla v(x)+\frac{\partial f}{\partial u}(x, u(x), \nabla u(x)) v(x)\right| \\
& \leq\left|\frac{\partial f}{\partial p}(x, u(x), \nabla u(x))\right||\nabla v(x)|+\left|\frac{\partial f}{\partial u}(x, u(x), \nabla u(x))\right||v(x)| \\
& \leq M(|v(x)|+|\nabla v(x)|), \quad x \in \Omega,
\end{aligned}
$$

para alguma constante $M>0, f$ é de classe $C^{2}$. Recorremos uma vez mais ao Teorema 1.2 para inferir que $v=0$.

Portanto, $\frac{\partial G}{\partial u}\left(u, i_{\Omega}\right)$ é semi-Fredholm, com índice $\leq 0$. Quanto à verificação de que $G$ satisfaz a hipótese (2)(b) do Corolário 3.7 , mostraremos que o espaço $\mathcal{R}\left(D G\left(u, i_{\Omega}\right)\right) / \mathcal{R}\left(\frac{\partial G}{\partial u}\left(u, i_{\Omega}\right)\right)$ tem dimensão infinita, argumentando por contradição.

Suponha que

$$
m=\operatorname{dim}\left\{\mathcal{R}\left(D G\left(u, i_{\Omega}\right)\right) / \mathcal{R}\left(\frac{\partial G}{\partial u}\left(u, i_{\Omega}\right)\right)\right\}<\infty
$$

Levando em consideração que $D G\left(u, i_{\Omega}\right): W_{0}^{2, p}(\Omega) \times C^{3}\left(\Omega, \mathbb{R}^{n}\right) \longrightarrow L^{p}(\Omega)$ é dada por

$$
D G\left(u, i_{\Omega}\right)(\dot{u}, \dot{h})=L_{u}(\dot{u}-\dot{h} \cdot \nabla u)
$$

deduzimos que existem $\left\{f_{1}, \ldots, f_{m}\right\}$ em $L^{p}(\Omega)$, com $f_{i}=L_{u}\left(\dot{u}_{i}-\dot{h}_{i} \cdot \nabla u\right), \dot{u}_{i} \in W_{0}^{2, p}(\Omega), \dot{h}_{i} \in$ $C^{3}\left(\Omega, \mathbb{R}^{n}\right), i=1, \ldots, m$, tais que para todo $(\dot{u}, \dot{h}) \in W_{0}^{2, p}(\Omega) \times C^{3}\left(\Omega, \mathbb{R}^{n}\right)$ existem $\dot{v} \in W_{0}^{2, p}(\Omega)$ e únicos $c_{1}, \ldots, c_{m} \in \mathbb{R}$ cumprindo

$$
\sum_{i=1}^{m} c_{i} f_{i}+L_{u} \dot{v}=L_{u}(\dot{u}-\dot{h} \cdot \nabla u)
$$

ou seja,

$$
\sum_{i=1}^{m} c_{i} L_{u}\left(\dot{u}_{i}-\dot{h}_{i} \cdot \nabla u\right)+L_{u} \dot{v}-L_{u}(\dot{u}-\dot{h} \cdot \nabla u)=0
$$

resultando que

$$
L_{u}\left(\dot{u}-\dot{v}-\sum_{i=1}^{m} c_{i} u_{1}-\left(\dot{h}-\sum_{i=1}^{m} c_{i} \dot{h}_{i}\right) \cdot \nabla u\right)=0
$$

Se $\left\{\phi_{1}, \ldots, \phi_{k}\right\}$ é uma base do operador de Fredholm $L_{u}: W^{2, p} \cap W_{0}^{1, p}(\Omega) \longrightarrow L^{p}(\Omega)$, temos

$$
\dot{u}-\dot{v}-\sum_{i=1}^{m} c_{i} \dot{u}_{i}-\left(\dot{h}-\sum_{i=1}^{m} c_{i} \dot{h}_{i}\right) \cdot \nabla u=\sum_{j=1}^{k} b_{j} \phi_{j}
$$

para certas constantes $b_{1}, \ldots, b_{k}$.

Já sabemos que $u \in W^{3, p} \cap W_{0}^{2, p}(\Omega)$, de modo que $\frac{\partial u}{\partial x_{i}} \in W^{2, p} \cap W_{0}^{1, p}(\Omega)$ para $i=1, \ldots, n$. 
Calculando a derivada normal da igualdade em (4.8) sobre $\partial \Omega$, obtemos que

$$
-\left(\dot{h}-\sum_{i=1}^{m} c_{i} \dot{h}_{i}\right) \cdot N \frac{\partial^{2} u}{\partial N^{2}}=\sum_{j=1}^{k} b_{j} \frac{\partial \phi_{j}}{\partial N} .
$$

Pelo Teorema 1.1 sabemos que $\Delta_{\partial \Omega} u=\Delta u-\operatorname{div}(N) \frac{\partial u}{\partial N}-\frac{\partial^{2} u}{\partial N^{2}}$. Como $u=0$ em $\partial \Omega$ temos que $\Delta_{\partial \Omega}=0$ e, por conseguinte, $\frac{\partial^{2} u}{\partial N^{2}}=\Delta u=-f(x, 0,0)$ sobre $\partial \Omega$. Portanto, sobre $\partial \Omega$,

$$
\dot{h} \cdot N f(x, 0,0)=\sum_{i=1}^{m} c_{i} \dot{h}_{i} \cdot N f(x, 0,0)+\sum_{j=1}^{k} b_{j} \frac{\partial \phi_{j}}{\partial N} .
$$

Acabamos de demonstrar que para cada $h \in C^{3}\left(\Omega, \mathbb{R}^{n}\right)$ existem constantes reais $c_{1}, \ldots, c_{m}$, $b_{1}, \ldots, b_{k}$ para as quais a igualdade (4.9) ocorre. Em outras palavras, provamos que o operador limitado

$$
\left.\dot{h} \longmapsto \dot{h} \cdot N f(x, 0,0)\right|_{\partial \Omega}: C^{3}\left(\Omega, \mathbb{R}^{n}\right) \longrightarrow C^{0}(\partial \Omega, \mathbb{R})
$$

tem imagem de dimensão finita, contrariando o fato de termos $\left.f(x, 0,0)\right|_{\partial \Omega} \neq 0$ e $\operatorname{dim} \partial \Omega \geq 1$.

Logo, a dimensão do espaço quociente $\mathcal{R}\left(D G\left(u, i_{\Omega}\right)\right) / \mathcal{R}\left(\frac{\partial G}{\partial u}\left(u, i_{\Omega}\right)\right)$ é infinita e a hipótese (2)(b) do Corolário 3.7 está satisfeita pela aplicação $G$.

Por fim, vejamos que a aplicação $(u, h) \longmapsto h: G^{-1}(0) \longrightarrow V_{h_{k}}$ é própria. Seja $\left\{\left(u_{n}, h_{n}\right)\right\}$ uma sequência em $G^{-1}(0)$ tal que $h_{n} \rightarrow h$ em $V_{h_{k}}$. Então

$$
\left\|\Delta h_{n}^{*-1} u_{n}\right\|_{L^{p}\left(h_{n}(\Omega)\right)}=\left\|f\left(\cdot, h_{n}^{*-1} u_{n}, \nabla h_{n}^{*-1} u_{n}\right)\right\|_{L^{p}\left(h_{n}(\Omega)\right)} \leq M
$$

para alguma constante $M>0$, já que $f$ é limitada e $\left\{m\left(h_{n}(\Omega)\right)\right\}$ é uma sequência limitada. Como $\Delta$ é um isomorfismo, sabemos que passando a uma subsequência, $\left\{\left\|h^{*-1} u_{n}\right\|_{W_{0}^{2, p}\left(h_{n}(\Omega)\right)}\right\}$ converge.

Usando o fato de que $\left\{h_{n}^{*}\right\}$ é uma sequência de isomorfismos que converge para $h^{*}$, concluímos que

$$
\left\|h^{*-1} u_{n}\right\|_{W^{2, p}\left(h_{n}^{*}(\Omega)\right)} \geq m_{n}\left\|u_{n}\right\|_{W^{2, p}\left(h_{n}^{*}(\Omega)\right)} \geq m\left\|u_{n}\right\|_{W_{0}^{2, p}(\Omega)},
$$

para todo $n \in \mathbb{N}$, de maneira que $\left\{u_{n}\right\}$ é uma sequência limitada em $W_{0}^{2, p}(\Omega)$. Mas, na verdade, por ser $\Omega$ uma região $C^{3}$-regular, $\left\{u_{n}\right\}$ é uma sequência limitada em $W^{3, p} \cap W_{0}^{2, p}(\Omega)$. A imersão compacta de $W^{3, p}(\Omega)$ em $W^{2, p}(\Omega)$ garante a existência de uma subsequência de $\left\{u_{n}\right\}$ que converge em $W_{0}^{2, p}(\Omega)$. Logo, $(u, h) \longmapsto h: G^{-1}(0) \longrightarrow V_{h_{k}}$ é aplicação própria.

Podemos então aplicar o Corolário 3.7 para obter um subconjunto $O \subset V_{h_{k}}$, fechado e com interior vazio, tal que $0 \notin G\left(W_{0}^{2, p}(\Omega), h\right)$ sempre que $h \in V_{h_{k}} \backslash O$, como pretendíamos.

Considerando $\tilde{F}: W^{2, p} \cap W_{0}^{1, p}(\Omega) \times\left(V_{h_{k}} \backslash O\right) \rightarrow L^{p}(\Omega)$ a restrição de $F$ ao conjunto $W^{2, p} \cap$ $W_{0}^{1, p}(\Omega) \times\left(V_{h_{k}} \backslash O\right)$, observamos que $\tilde{F}$ satisfaz as hipóteses (1), (2)(a) e (3) do Teorema 3.3 em todos os pontos de $\bar{F}^{-1}(0)$. Sendo assim, sabemos que existe um subconjunto magro $\tilde{H}_{k} \subset$ $V_{h_{k}} \backslash O$, com a propriedade de que zero é valor regular de $F_{h}$ para todo difeomorfismo $h \in$ $V_{h_{k}} \backslash\left(O \cup \tilde{H}_{k}\right)$. Fazendo $H_{k}=O \cup \tilde{H}_{k}$ chegamos ao subconjunto magro requerido. 


\section{Referências Bibliográficas}

[1] R. Abraham E J. RoBin, Transversal Mappings and flows, W. A. Benjamin, New York, 1967.

[2] S. Agmon, The $L^{p}$ approach to the Dirichlet problem, Ann. Scuola Norm. Sup. Pisa, 13 (1959), 405-448.

[3] S. Agmon, A. Douglis E N. NiRENBerg, Estimates near the boundary for solutions of elliptic partial differential equations satisfying general boundary conditions, Comm. Pure Appl. Math., 12 (1959), 623-727.

[4] S. B. ANGENENT, The Morse-Smale property for a semilinear parabolic equation, Journal of Differential Equations, 62 (1986), 427-442.

[5] H. Brezis, Analyse fonctionelle. Théorie et applications, Masson, Paris, 1987.

[6] P. BRunovskÝ AND S. N. Chow, Generic properties of stationary solutions of Reaction diffusion equations, J. Differential Equations, 53 (1984), 1-23.

[7] P. BRUNovsKÝ AND P. POLÁC̆IK, The Morse-Smale structure of a generic reactiondiffusion equation in higher space dimension, J. Differential Equations, 135 (1997), 129-181.

[8] D. Gilbarg E N. S. TRUdinger, Elliptic partial diferential equations of second order, Springer Verlag, Berlin, 1983.

[9] J. K. Hale, L. T. Magalhães and W. M. Oliva, An Introduction to infinite dimensional dynamical systems - Geometric theory, Springer-Verlag, New York, 1984.

[10] D. HENRY, Geometric theory of semilinear parabolic equations, Lecture Notes in Mathematics 840, Springer-Verlag, Berlin, 1981.

[11] D. HENRY, Some Infinite-Dimensional Morse-Smale Systems Defined by Parabolic Partial Differential Equations, Journal of Differential Equations, 59 (1985).

[12] D. HENRY, Pertubation of the boundary for boundary value problems of partial differential operators, Notas mimeografadas, IME-USP, Sāo Paulo, 1985. 
[13] L. HORMANDER, Linear partial differential operators, Springer Verlag, New York; 1964.

[14] T. Kato, Perturbation theory for linear operators, Springer Verlag, New York, 1980.

[15] A. M. Michelett1, Perturbazione dello spettro dell'operatore de laplace in relazione ad una variazone del campo, Ann. Scuola Norm. Sup. Pisa, 26 (1972), 151-169.

[16] P. POLÁčlK, Transversal and nontransversal intersections of stable and unstable manifolds in reaction and diffusion equations on symmetric domains, Differential and Integral Equations, 7 (1994), 1527-1545.

[17] C. RochA, Generic properties of equilibria of reaction-diffusion equations with variable diffusion, Proc. Roy. Soc. Edinburgh Ser A, 101 (1985), 45-55.

[18] J. SAUT AND R. TEMAM, Generic properties of nonlinear boundary value problems, Comm. Partial Differential Equations, 4 (1979), 293-319. 